\title{
AN INVESTIGATION OF THE ROLES OF GEOMAGNETIC AND ACOUSTIC CUES IN WHALE NAVIGATION AND ORIENTATION
}

By

Ann Nichole Allen

B.S. University of California, Santa Cruz, 2006

Submitted in partial fulfillment of the requirements for the degree of

Doctor of Philosophy

at the

MASSACHUSETTS INSTITUTE OF TECHNOLOGY
and the
WOODS HOLE OCEANOGRAPHIC INSTITUTION

June 2013

(C2013 Ann N. Allen.

All rights reserved.

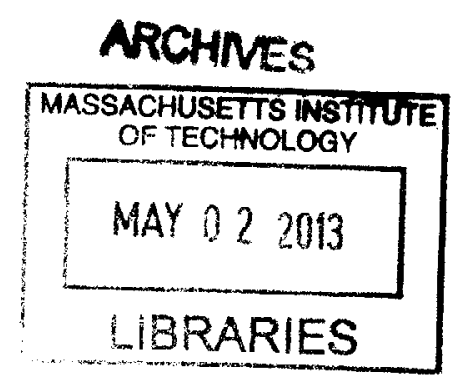

The author hereby grants to MIT and WHOI permission to reproduce and to distribute publicly paper and electronic copies of this thesis document in whole or in part in any medium now known or hereafter created.

Author

Joint Program in Oceanography and Applied Ocean Science and Engineering Massachusetts Institute of Technology and Woods Hole Oceanographic Institution

Certified by

May 3, 2013

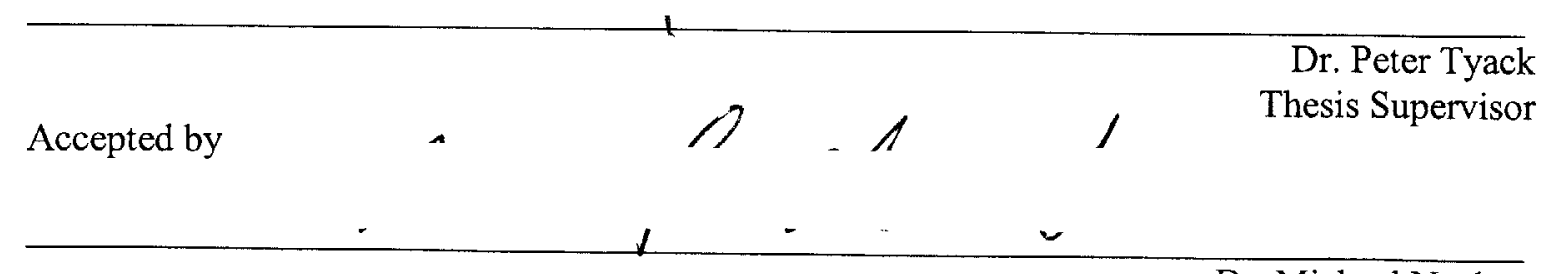

Dr. Michael Neubert Chair, Joint Committee for Biological Oceanography Woods Hole Oceanographic Institution 


\title{
An investigation of the roles of geomagnetic and acoustic cues in whale navigation and orientation
}

\author{
By \\ Ann Nichole Allen \\ Submitted to the MIT/WHOI Joint Program in Oceanography and Applied Ocean Science and \\ Engineering on May 3, 2013 in partial fulfillment of the requirements for the degree of Doctor of \\ Philosophy in the field of Biological Oceanography
}

\begin{abstract}
Many species of whales migrate annually between high-latitude feeding grounds and low-latitude breeding grounds. Yet, very little is known about how these animals navigate during these migrations. This thesis takes a first look at the roles of geomagnetic and acoustic cues in humpback whale navigation and orientation, in addition to documenting some effects of human-produced sound on beaked whales.

The tracks of satellite-tagged humpback whales migrating from Hawaii to Alaska were found to have systematic deviations from the most direct route to their destination. For each whale, a migration track was modeled using only geomagnetic inclination and intensity as navigation cues. The directions in which the observed and modeled tracks deviated from the direct route were compared and found to match for 7 out of 9 tracks, which suggests that migrating humpback whales may use geomagnetic cues for navigation. Additionally, in all cases the observed tracks followed a more direct route to the destination than the modeled tracks, indicating that the whales are likely using additional navigational cues to improve their routes.

There is a significant amount of sound available in the ocean to aid in navigation and orientation of a migrating whale. This research investigates the possibility that humpback whales migrating near-shore listen to sounds of snapping shrimp to detect the presence of obstacles, such as rocky islands. A visual tracking study was used, together with hydrophone recordings near a rocky island, to determine whether the whales initiated an avoidance reaction at distances that varied with the acoustic detection range of the island. No avoidance reaction was found. Propagation modeling of the snapping shrimp sounds suggested that the detection range of the island was beyond the visual limit of the survey, indicating that snapping shrimp sounds may be suited as a long-range indicator of a rocky island.

Lastly, this thesis identifies a prolonged avoidance reaction of a Blainville's beaked whale to playbacks of Navy mid-frequency active sonar and orca predation calls. Navy sonar exercises have been linked to beaked whale strandings, and identifying whether these are the result of a mistaken predator avoidance reaction may help prevent future strandings.
\end{abstract}

Thesis supervisor: Dr. Peter Tyack

Title: Professor, Scottish Oceans Institute, School of Biology, University of St Andrews 


\section{Acknowledgements}

This thesis would not have been possible without the support of innumerable individuals who have contributed their time and energy over the last five and a half years. I would like to first thank my advisor, Peter Tyack. He has been so amazingly supportive during my time at WHOI, allowing me to pursue the project that most interested me. His ability to focus on the positive and always find the important and exciting aspects of a project astounds me, and was essential in providing me with the motivation and excitement to persevere through graduate school. Additionally, he provided continual guidance and assistance through all aspects of my journey to becoming a scientist.

The other members of my committee were essential in providing support and insights along the way. Mark Baumgartner and Jim Lynch in particular dedicated large amounts of their time to preparing me for field projects as well as aiding in analysis of the data. John Leonard and Glen Gawarkiewicz also dedicated significant amounts of their time to seeing me through this process. Laela Sayigh was kind enough to chair my thesis defense, as well as providing mentorship and insight throughout my time at WHOI.

Alex Bocconcelli provided invaluable support and practical advice in preparing for field projects. The work in Australia would not have been possible without the assistance of Keith Von Der Heydt, Mike Noad, Rebecca Dunlop, Doug Cato, Michael Phillips and numerous volunteers. Bruce Mate generously shared the data he collected in Hawaii for the analysis in Chapter 2. In addition, many individuals aided in the analysis of the data in this thesis including: Art Newhall, Ben Hodges, Andy Solow and Julian Schanze.

The past and present members of the WHOI Academic Programs Office deserve many thanks. They do a wonderful job supporting all students throughout their time here, and go above and beyond in their devotion to getting us all through the program.

In addition to all of the professional support I have received along the way, I would not have been able to make this journey without the support of friends and family. I would like to particularly thank my housemates Kim Popendorf and Maya Yamato, who shared the entire journey through grad school with me and without whom this process would have been almost impossible. In addition, LiLing Hamaday, Carly Buchwald and too many others to list have provided huge amounts of support, distraction and commiseration throughout the years. Kara Hamilton has provided amazing support from afar, always seeming to sense when flowers or chocolate were needed, as well as reading over 100 pages of this thesis.

I would not be here without the support of my family. My parents always told me I could do whatever I wanted in life and fostered a love of learning in me at a young age. My grandmother supported me unconditionally in everything I wanted to do, and was always so proud of me. My grandfather, brother and countless other family members have always been encouraging and supported me throughout a lifetime of learning.

This work was funded by the Office of Naval Research, the Naval Postgraduate School, the Naval Environmental Readiness Division, the WHOI Academic Programs Office, the WHOI Ocean Ventures Fund the WHOI Coastal Ocean Institute and the National Geographic Committee for Research and Exploration. 


\section{TABLE OF CONTENTS}

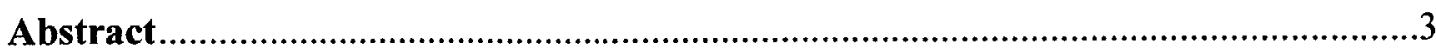

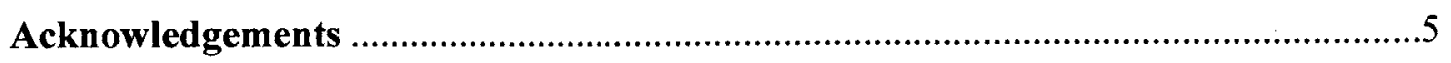

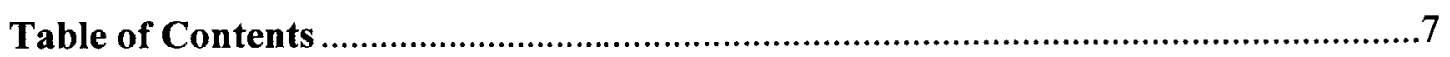

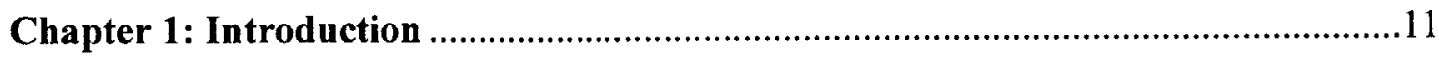

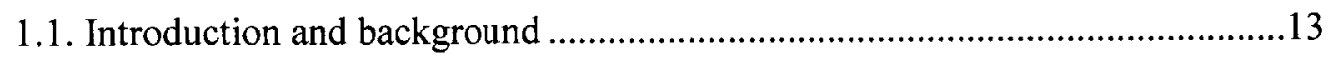

1.1.1. Navigation methods ..................................................... 14

1.1.2. Available navigation cues .................................................... 15

1.1.3. Geomagnetic cue use in navigation .......................................... 16

1.1.4. Acoustic cue use in navigation................................................ 18

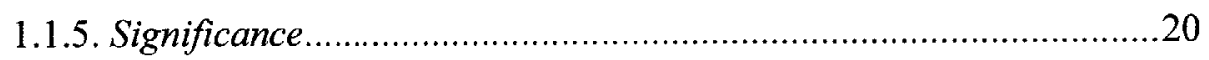

1.1.6. Summary of work presented ...............................................21

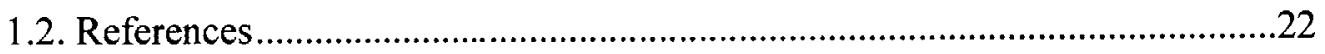

Chapter 2: Analysis of geomagnetic inclination and intensity as navigation cues in

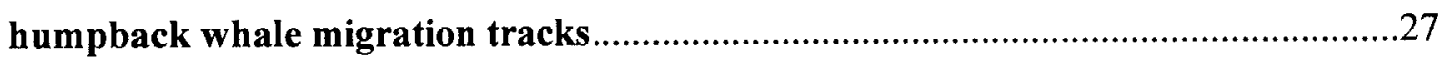

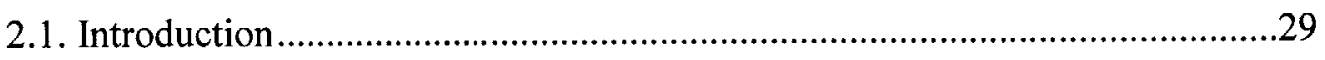

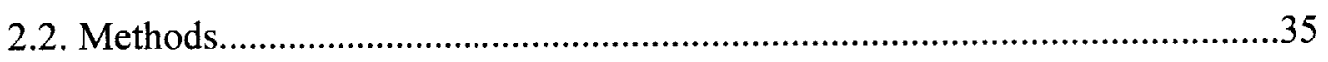

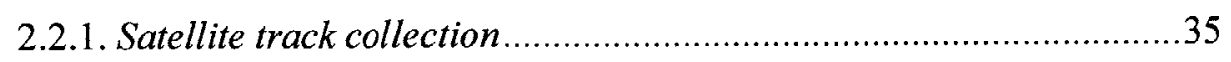

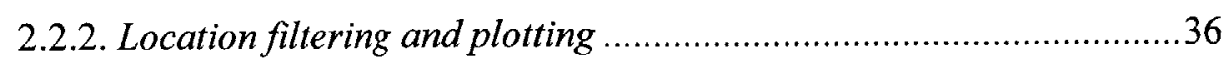

2.2.3. Modeling of bicoordinate geomagnetic navigation track ................37

2.2.4. Binomial test ................................................................... 40

2.2.5. Testing of model calculations utilizing simulated field

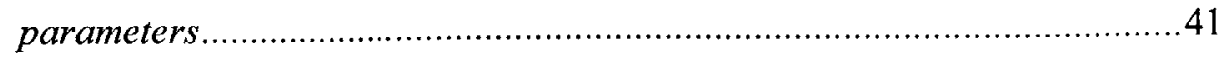

2.2.6. Accounting for surface ocean currents ..................................... 42 
2.2.7. Test for bearing correction.........................................................43

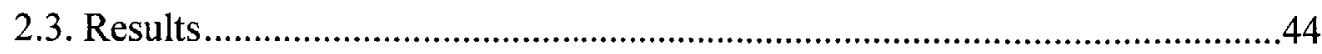

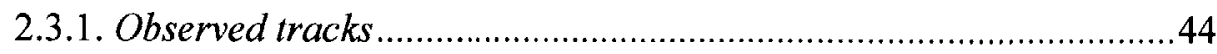

2.3.2. Comparison of bicoordinate geomagnetic tracks to observed

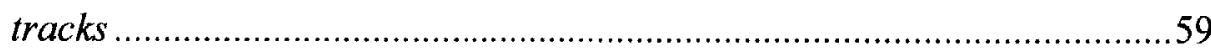

2.3.3. Bicoordinate tracks for simulated fields ...................................61

2.3.4. Surface currents and heading adjustments .................................66

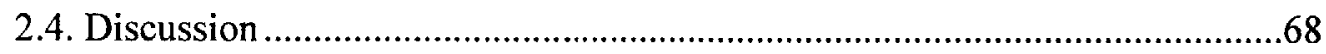

2.4.1. General navigation performance ...........................................68

2.4.2. Correlation between modeled and observed deviation directions ....70

2.4.3. Routes modeled with simulated field parameters............................71

2.4.4. Use of multiple cues during migration ........................................73

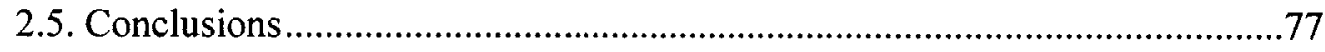

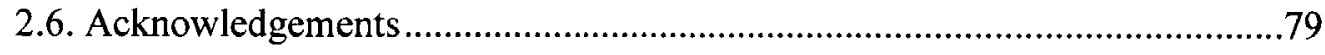

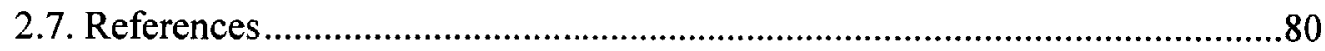

\section{Chapter 3: Snapping shrimp sounds as a potential acoustic orientation cue for}

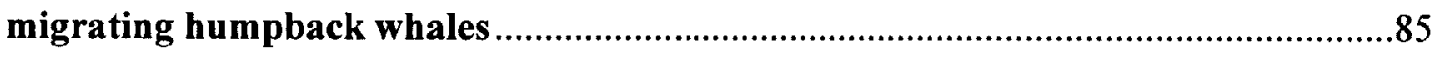

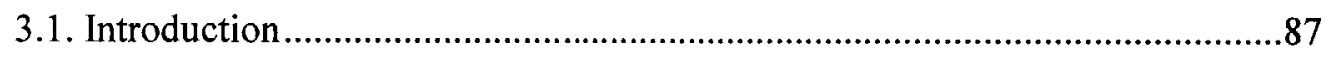

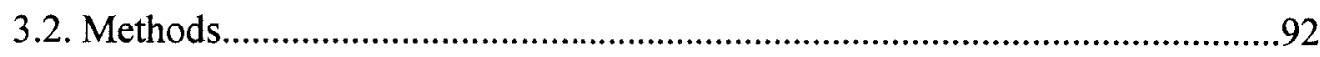

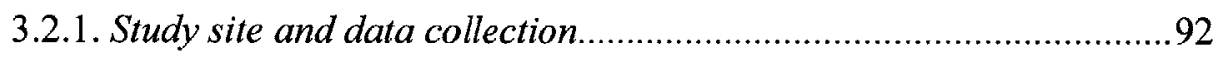

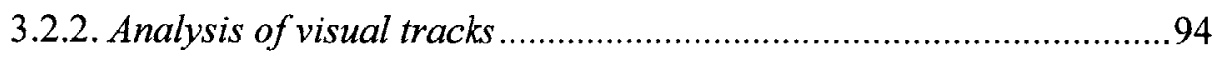

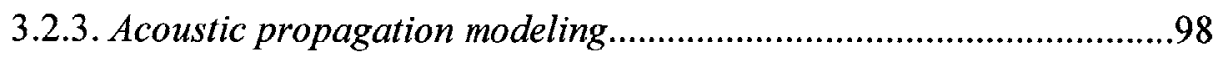

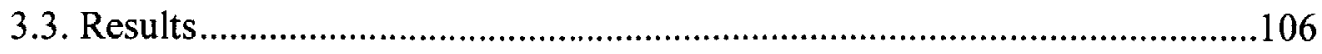

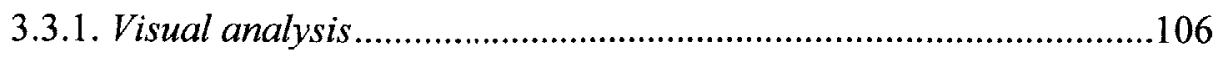

3.3.2. Acoustic modeling results ......................................................... 112 


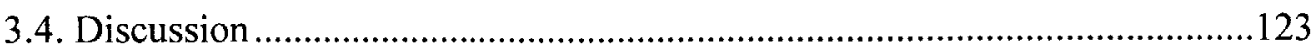

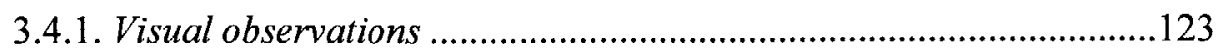

3.4.1. Sound field modeling of Boat Rock .................................................126

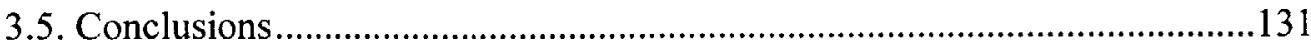

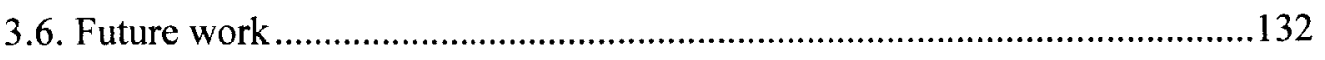

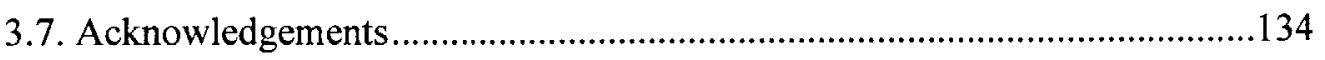

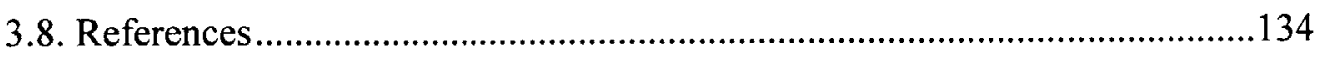

Chapter 4: Analysis of a Blainville's beaked whale's movement response to playback

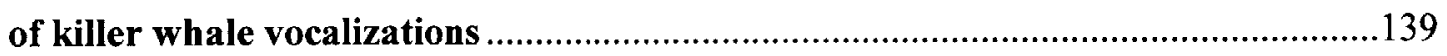

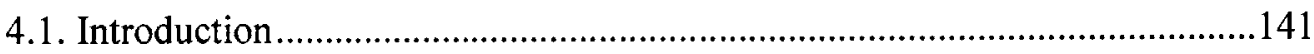

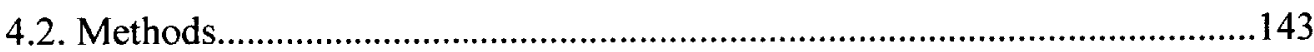

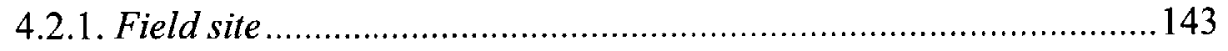

4.2.2. Digital acoustic recording tagging ................................................. 144

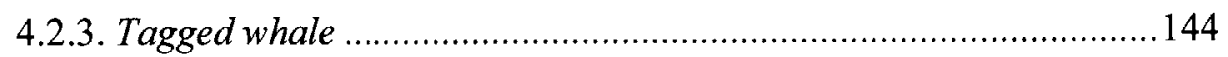

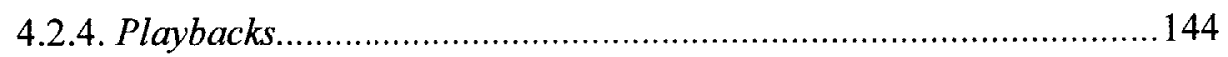

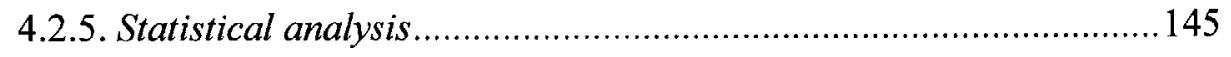

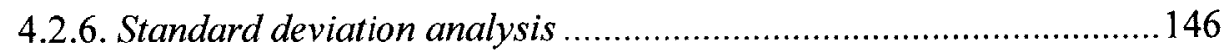

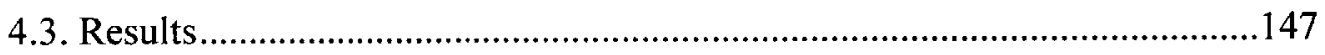

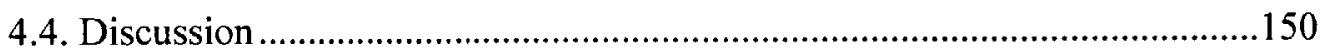

4.5. Acknowledgements....................................................................................153

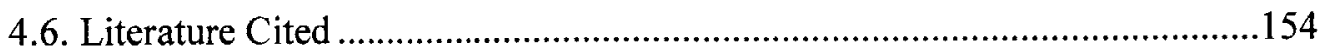

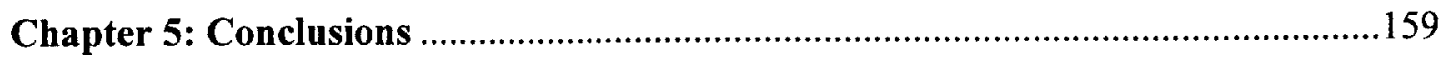




\section{Chapter 1}

Introduction 


\subsection{Introduction and background}

Cetaceans are one of only two orders of mammals that spend their entire lives in the water, an environment where visual and other typically thought of orientation cues are often unavailable. To effectively find food, breed, socialize and migrate in this environment they must have highly adapted sensory abilities. A wide variety of unique adaptations have been documented for these animals, most notably the use of high frequency sounds by odontocetes for echolocation. However, one area of their ecology that has been left relatively unaddressed is the extreme long distance migrations that most species of baleen whales perform annually.

The migrations of baleen whales include the longest known annual movements of any mammal, and yet they remain poorly understood (Corkeron and Connor 1999). Populations of baleen whales exist in all oceans of the world and most undertake an annual migration between summer high latitude feeding areas and winter low latitude breeding and calving grounds. While some of these migrations occur over the continental slope, the majority of populations traverse wide stretches of open ocean during their annual trips (Norris 1967). The open ocean is typically thought of as an environment lacking in any conventional navigation cues. Up until the recent invention of the Global Positioning System (GPS), human mariners often faced the very real possibility of becoming lost at sea during ocean crossings.

Yet in spite of the apparent homogeneity of the ocean, whales still manage to quickly and accurately cross large, open expanses of ocean on a yearly basis. Despite appearances, the natural marine environment is in fact filled with many potential sources of navigational information. There has been much speculation as to which mechanisms whales may be using to navigate, but there is still very little concrete information on the subject. With the advent of new tracking technology, and many examples of similar research conducted on migratory birds and sea turtles, an initial study of this subject is now a realistic prospect. 


\subsubsection{Navigation methods}

There are several different methods of navigation that migratory animals can employ: the first is stepping-stone or landmark navigation, in which travel is via several specific destinations or stop-over points before reaching a final destination; the second is vector navigation, in which the animal follows a "compass" bearing, or other direction value, for a specific period of time or distance in order to reach a migratory goal (e.g. head North in the spring); the third is true navigation by which the animal navigates directly to a goal even if displaced or side-tracked (Wiltschko and Wiltschko 2003, Bingman and Cheng 2005). Animals capable of true navigation, or homing, must possess a "map" as well as a "compass" sense (Lohmann et al. 2008). The map sense is used to determine the animal's position relative to its destination and the compass sense is used to determine the appropriate heading towards the goal from its current position (Lohmann et al. 2008). A simple directional sense cannot detect or correct for deviations from an intended course, and therefore even a small deflection could lead to a large error at the final destination. Surface currents in the ocean are a constant source of deflection that can push a marine animal off course. This is potentially acceptable if an animal is navigating to a large feature, such as a coastline, and it could still reach its destination in the face of an error. However, when navigating towards a specific destination, such as an oceanic island, an error could result in missing the destination entirely. Therefore, in order to accurately navigate to precise destinations within the ocean, a whale must possess some form of true navigation.

All migratory animals studied thus far employ multiple sources of information while navigating and can likely switch between them as needed (Lohmann et al. 2008), using one set of cues when moving over long distances, and another for localization of a particular target area once they approach their final destination (Bingman and Cheng 2005). There is increasing evidence that migratory animals use navigation cues in a mixture of both sequential and parallel combination (Bingman and Cheng 2005). The inherent variation, or unavailability, of certain cues over time makes a system of calibration, by comparing one cue against another, a highly desirable ability (Gould 
1998). An animal would benefit from having several different ways to maintain headings, in the event that one sense is impaired or one type of cue is unavailable. In addition, navigation cues or strategies may change depending on the range of the animal from the destination, as some indicators of location may be limited in their effectiveness at great distance from the destination. It is probable that migratory animals exploit all information available to them to make an informed decision on the best path to reach their destination. There has been an extensive amount of study focused on determining precisely what navigational cues migratory animals use and how they switch between them.

\subsubsection{Available navigation cues}

Many studies have shown that one of the primary ways by which animals orient and navigate in the natural environment is through use of the sun and other celestial cues. The sun can be used as a compass when its position over the course of the day is combined with an animal's internal clock ( $\AA$ kesson and Hedenström 2007). Experiments with shifting the internal clocks of birds have shown that this method of sun compass navigation is utilized by many different species (Wiltschko and Wiltschko 2003). Sea turtles use the sun's position to orient towards the ocean after hatching (Avens and Lohmann 2003), and it has been suggested that adult sea turtles use a sun compass in long distance navigation (Lohmann et al. 2008). Even insects employ celestial cues, with bees using patterns of sunlight polarization to navigate back to their hive (Rossel 1993), and dung beetles maintaining an orientation relative to the Milky Way in order to steer a straight line away from a dung pile (Dacke et al. 2013). Harbor seals have also been trained to use the stars to navigate in a controlled environment (Mauck et al. 2008), suggesting that marine mammals can potentially orient via celestial cues.

There is also evidence that chemosensory cues may play a role in navigation for both birds and sea turtles (Wallraff 2004, Lohmann et al. 2008). Some migrating seabirds have shown a sensitivity to dimethyl suphide (DMS), a scented compound produced by phytoplankton and released to the atmosphere (Nevitt and Bonadonna 2005). 
Spatial patterns of DMS are known to reflect bathymetric and oceanic features such as shelf breaks, sea mounts and ocean frontal zones, and thus could be used as a cue to the presence of these features (Nevitt and Bonadonna 2005). Additionally, several studies have hypothesized that turtles may memorize the chemosensory information from the chemical plumes of an island or river outlet at their hatching site and can later follow these plumes back to their natal sites (Lohmann et al. 2008). While chemosensory cues could provide information very specific to a site, they are limited in their detectible range.

In addition, there are a wide range of physical features of the ocean that could provide useful navigation information. Despite the apparent homogeneity of the open ocean, many scales of structure in the physical environment can be used by an organism to locate and maintain its position (Kobayashi et al. 2008). The North Pacific has shifting structures such as fronts, eddies, and jets. An oceanic front is a narrow zone of enhanced horizontal gradients of water properties, such as temperature, salinity, and nutrients, that occur on varying length scales, from a few meters to thousands of kilometers (Belkin et al. 2009). Some fronts can be quasi-stationary and seasonally persistent (Belkin et al. 2009), representing a seasonal, stationary cue that migrating animals could utilize to aid in navigation. Other large scale oceanographic indicators of direction include water masses or currents that might serve as signposts (Norris 1967), and sea surface temperature (SST), which varies greatly with latitude and could provide a reliable, albeit coarse resolution, indication of direction.

In addition to large scale oceanographic features, waves are generally refracted as they pass around islands, creating characteristic interference patterns that could be detected by the accelerations and water movements associated with the waves (Lohmann et al. 2008). These operate on a local scale and would be very specific to a destination.

\subsubsection{Geomagnetic cue use in navigation}

While there is a huge variety of cues that can provide a migrating animal with orientation information, the one that is widely agreed to be the most useful to a navigating animal is the earth's magnetic field. The direction of the magnetic field lines 
could be used by an animal, in the same way that humans use them, in order to get a compass bearing. However, what makes the geomagnetic field most suited as a navigational cue is that it could potentially form a bicoordinate magnetic 'map' for position finding. At each location on the globe the geomagnetic field lines intersect the surface of the earth at a specific angle of inclination. This angle varies with latitude, so an animal that can distinguish between different field inclinations could potentially determine its approximate latitude (Lohmann et al. 1999). In addition, the total intensity of the magnetic field varies over the surface of the earth, and could be used with inclination to assess position relative to other combinations of these field values.

The importance of the geomagnetic field in animal navigation is evidenced by the large number of species that have been shown to use it thus far. Extensive studies of both birds and sea turtles have shown that the earth's magnetic field is essential to their navigational performance (Papi et al. 1995, Luschi et al. 1998, Lohmann et al. 2001, Wiltschko and Wiltschko 2003). Additionally, a magnetic sense has been found in a wide variety of taxa, including bats, spiny lobsters, hamsters, mole rats and even newts (Marhold et al. 1997, Fischer et al. 2001, Boles and Lohmann 2003, Deutschlander et al. 2003, Cochran et al. 2004, Holland et al. 2006). While there is no overall agreement on the way that these animals detect and navigate via the geomagnetic field, the fact that a magnetic sense is present across so many species indicates that it may be an essential component to successful navigation and orientation.

While there has been some effort to determine if cetaceans possess such a magnetic sense, there have been no conclusive studies on the subject. There are some correlations of whale stranding events with geomagnetic anomalies, as well as associations of fin whale sightings with geomagnetic minima (Kirschvink et al. 1986, Klinowska 1986, Walker et al. 1992). Yet despite agreement that such a sense would be exceedingly beneficial to a migrating whale, the difficulty of performing any experimental tests makes conclusively demonstrating geomagnetic sensitivity in cetaceans extremely difficult. However, the widespread presence of a geomagnetic sense 
in many diverse species of long-distance migrators suggests that it could also be an important cue for whales and is a worthwhile area of investigation.

\subsubsection{Acoustic cue use in navigation}

One of the sensory cues that has particular importance in a marine environment is sound. In an environment where vision is frequently limited, sound, particularly lowfrequency (LF) sound has the potential to provide information over great distances. The vocalizations and hearing capabilities of baleen whales are particularly adapted to LF sound generation and reception (Wartzok and Ketten 1999), indicating that these animals are already attuned to such information in the marine environment.

It has often been theorized that baleen whales may actively listen to echoes from their vocalizations to gain information about the environment (George et al. 1989). Frazer and Mercado (2000) developed a model suggesting that male humpback whales could utilize their songs to locate females on the breeding grounds, and while other sources claim there are no indications this does in fact occur (Au et al. 2001), there has been no concrete resolution to this debate. Recent research has also shown that some humpback whales emit LF broadband 'clicks', termed megapclicks, while foraging at night (Stimpert et al. 2007). It was speculated that these clicks may be used for acoustic detection purposes, such as identifying the seafloor or other large, nearby targets.

It has also been hypothesized that arctic baleen whales can use acoustic cues to detect ice presence and thickness (Ellison et al. 1987, George et al. 1989). In the case of the bowhead whale, they must break through the ice in order to obtain breathing holes, therefore assessing ice thickness is a life-or-death problem. Ellison et al. (1987) suggest that bowhead whales can assess these ice conditions using their vocalizations. Their calculations show that a bowhead could determine where the ice is thin or thick by the relative levels of echoes of its own calls from the ice. This could allow the whales to differentiate between thick pack ice and thin new ice that could be broken for breathing holes. George et al. (1989) also found that the whales deflect around multi-year ice flows 
that would be too thick to break through. They suggest that the whales find breathing holes through a combination of visual and acoustic cues.

In addition to the active use of their vocalizations, there is a significant amount of noise generated in the ocean just by the breaking of shore waves that could be useful to a migrating whale. Long term observations of shore noise show that breaking waves generate sound between 100 and $1,000 \mathrm{~Hz}$, which is within the ideal inferred hearing range of humpback whales, and that noise levels scale with the square of surface wave height (Deane 2000). Many areas also experience seasonal variability in ambient sound levels due to seasonal storms (Curtis et al. 1999). This low frequency noise may propagate long distances with very little attenuation, creating patterns of infrasound in the sea. Evidence from homing pigeons suggests that they may use infrasonic cues generated by oceanic waves to navigate (Hagstrum 2000). Atlantic cod have also been shown to have a sensitivity to infrasound that may be employed for orientation during their migration (Sand and Karlsen 1986). It has also been suggested that grey whales may listen to sounds from breaking waves during their migration along the California coast, keeping the sound of the surf to one side to maintain their direction (Mate and UrbanRamirez 2003).

In addition to sounds from physical factors, the whales may also be able to pick up social sounds from migrating conspecifics to indicate that they are moving in the correct direction. An acoustic survey of the North Pacific found large groupings of acoustic detections of humpback whales, which were attributed to a dispersed group of singing animals migrating within a few tens of miles of each other (Norris et al. 1999). While the whales may be vocalizing for social communication, other whales could benefit from eavesdropping on these calls in order to gain information on migration corridors.

Furthermore, there is a large amount of biological sound produced by other species in the ocean that could be useful to a migrating whale. Fish, snapping shrimp, and other invertebrates generate a significant amount of noise in certain areas. A combination of biologically produced sounds could serve as signposts for particular 
areas, indicating not only depth, but ecological factors such as substrate type (Norris 1967). Larval fish and invertebrates have been shown to use biological sounds to assess the suitability of settling sites and navigate to these locations (Simpson et al. 2005). With so much oceanic noise present, at both long and short distance scales, it is reasonable to explore whether migrating whales explore their environment through this acoustic information.

\subsubsection{Significance}

The problem of how migratory animals navigate has long been a subject of curiosity and investigation. We are only beginning to understand what cues are involved in navigation and orientation, and a whole new suite of sensory mechanisms has been revealed by studying animal navigational abilities, the most prominent of which is the magnetic sense discovered in birds, sea turtles, and other animals. If this, or any other directional sense, could be conclusively found in baleen whales, it would have widespread implications for the behavior, ecology, and biology not only of cetaceans, but of mammals in general. Information on marine mammal navigational cues would spur new insights and studies into the vocalizations, behavioral ecology, and physiology of this diverse group of animals.

Aside from the purely intellectual merits of such a study, a discovery of orientation mechanisms in whales would have impacts on marine mammal conservation. Human sources of noise in the ocean have far ranging effects on cetaceans ranging from temporary avoidance of an area, habitat desertion, temporary hearing loss, and even stranding and death. If acoustic cues are found to be important in baleen whale navigation it would provide valuable information that could help shape international policy on anthropogenic noise impacts on the ocean. Additionally, many apparently healthy marine mammals live strand on beaches every year, particularly in specific problem areas. It has been suggested that these strandings are the result of a navigational error. If this is the case, then understanding what navigate cues are important to 
migrating cetaceans could aid in the development of technology to divert animals around these areas and prevent future stranding events.

\subsubsection{Summary of work presented}

This study aims to look at several different navigation and orientation cues that could be utilized by migrating whales, from the spatial scales of a few meters to thousands of kilometers. Humpback whales (Megaptera novaeangliae) are among the best studied of the baleen whales, with well known populations on both their breeding and feeding grounds, as well as a growing number of animals that have been tracked during their migration. Therefore most of this work focuses on studying navigation and orientation in this relatively more accessible species of whale.

Chapter 2 of this thesis examines the tracks of humpback whales satellite tagged during their migrations from Hawaii to Alaska to determine the importance of geomagnetic cues in long distance navigation. This chapter focuses on comparison of the observed satellite tracks of these whales with migration paths modeled according to navigation solely via geomagnetic inclination and intensity.

Chapter 3 is a study of the role of acoustic cues in short range obstacle avoidance of humpback whales migrating near the Australian coastline. This portion of the study focuses mainly on biologically generated sounds from snapping shrimp on a rocky island, estimates the range at which they can be detected by an approaching whale, and assesses the plausibility of whether or not they can serve as an indicator of an island's presence.

Chapter 4 demonstrates the effects of certain kinds of anthropogenic sounds on marine mammals, specifically Navy mid-frequency active sonar which has been correlated with the stranding deaths of beaked whales. This chapter utilizes changes in movement patterns of a tagged beaked whale after playback of Navy sonar and killer whale predation calls to quantify the reaction of the whale to these sounds. This chapter was crucial in developing several of the movement analysis techniques utilized in the first two data chapters. 
Cumulatively, this thesis takes a first look at the plausibility of geomagnetic and acoustic cue use in humpback whale navigation as well as exploring some of the negative effects human generated sound can have on cetaceans.

\subsection{References}

Åkesson, S. and A. Hedenström. 2007. How migrants get there: migratory performance and orientation. BioScience 57:123-133.

Au, W. W. L., A. Frankel, D. A. Helweg and D. H. Cato. 2001. Against the humpback whale sonar hypothesis. IEEE Journal of Oceanic Engineering 26:295-300.

Avens, L. and K. J. Lohmann. 2003. Use of multiple orientation cues by juvenile loggerhead sea turtles Caretta caretta. Journal of Experimental Biology 206:4317-4325.

Belkin, I. M., P. C. Cornillon and K. Sherman. 2009. Fronts in large marine ecosystems. Progress In Oceanography 81:223-236.

Bingman, V. P. and K. Cheng. 2005. Mechanisms of animal global navigation: comparative perspectives and enduring challenges. Ethology, Ecology \& Evolution 17:295.

Boles, L. C. and K. J. Lohmann. 2003. True navigation and magnetic maps in spiny lobsters. Nature 421:60-63.

Cochran, W. W., H. Mouritsen and M. Wikelski. 2004. Migrating songbirds recalibrate their magnetic compass daily from twilight cues. Science 304:405-408.

Corkeron, P. J. and R. C. Connor. 1999. Why do baleen whales migrate? Marine Mammal Science 15:1228-1245.

Curtis, K. R., B. M. Howe and J. A. Mercer. 1999. Low-frequency ambient sound in the North Pacific: Long time series observations. The Journal of the Acoustical Society of America 106:3189-3200.

Dacke, M., E. Baird, M. Byrne, C. H. Scholtz and E. J. Warrant. 2013. Dung beetles use the milky way for orientation. Current Biology 23:298-300.

Deane, G. B. 2000. Long time-base observations of surf noise. The Journal of the Acoustical Society of America 107:758-770. 
Deutschlander, M. E., M. J. Freake, S. C. Borland, J. B. Phillips, R. C. Madden, L. E. Anderson and B. W. Wilson. 2003. Learned magnetic compass orientation by the Siberian hamster, Phodopus sungorus. Animal Behaviour 65:779-786.

Ellison, W. T., C. W. Clark and G. C. Bishop. 1987. Potential use of surface reverberation by bowhead whales, Balaena mysticetus, in under-ice navigation. Report of the International Whaling Commission 37:329-332

Fischer, J., M. Freake, S. Borland and J. Phillips. 2001. Evidence for the use of magnetic map information by an amphibian. Animal Behaviour 62:1-10.

Frazer, L. N. and E. Mercado III. 2000. A sonar model for humpback whale song. IEEE Journal of Oceanic Engineering 25:160.

George, J. C., C. W. Clark, G. M. Carroll and W. T. Ellison. 1989. Observations on the ice-breaking and ice navigation behavior of migrating bowhead whales (Balaena mysticetus) near Point Barrow, Alaska, Spring 1985. Arctic 42:24-30.

Gould, J. L. 1998. Sensory bases of navigation. Current Biology 8:R731-R738.

Hagstrum, J. T. 2000. Infrasound and the avian navigational map. The Journal of Experimental Biology 203:1103-1111.

Holland, R. A., K. Thorup, M. J. Vonhof, W. W. Cochran and M. Wikelski. 2006. Navigation: Bat orientation using Earth's magnetic field. Nature 444:702-702.

Kirschvink, J. L., A. E. Dizon and J. A. Westphal. 1986. Evidence from strandings for geomagnetic sensitivity in cetaceans. Journal of Experimental Biology 120:1-24.

Klinowska, M. 1986. Cetacean live stranding dates relate to geomagnetic disturbances. Aquatic Mammals 11:109.

Kobayashi, D. R., J. J. Polovina, D. M. Parker, N. Kamezaki, I. J. Cheng, I. Uchida, P. H. Dutton and G. H. Balazs. 2008. Pelagic habitat characterization of loggerhead sea turtles, Caretta caretta, in the North Pacific Ocean (1997-2006): Insights from satellite tag tracking and remotely sensed data. Journal of Experimental Marine Biology and Ecology 356:96-114.

Lohmann, K. J., J. T. Hester and C. Lohmann. 1999. Long-distance navigation in sea turtles. Ethology, Ecology \& Evolution 11:1-23.

Lohmann, K. J., S. D. Cain, S. A. Dodge and C. M. F. Lohmann. 2001. Regional magnetic fields as navigational markers for sea turtles. Science 294:364-366. 
Lohmann, K. J., P. Luschi and G. C. Hays. 2008. Goal navigation and island-finding in sea turtles. Journal of Experimental Marine Biology and Ecology 356:83-95.

Luschi, P., G. C. Hays, C. D. Seppia, R. Marsh and F. Papi. 1998. The navigational feats of green sea turtles migrating from Ascension Island investigated by satellite telemetry.

Proceedings of the Royal Society. Biological sciences 265:2279-2284.

Marhold, S., W. Wiltschko and H. Burda. 1997. A magnetic polarity compass for direction finding in a subterranean mammal. Naturwissenschaften 84:421-423.

Mate, B. R. and J. Urban-Ramirez. 2003. A note on the route and speed of a gray whale on its northern migration from Mexico to central California, tracked by satellitemonitored radio tag. The Journal of Cetacean Research and Management 5:155.

Mauck, B., N. Gläser, W. Schlosser and G. Dehnhardt. 2008. Harbour seals (Phoca vitulina) can steer by the stars. Animal Cognition 11:715-718.

Nevitt, G. A. and F. Bonadonna. 2005. Sensitivity to dimethyl sulphide suggests a mechanism for olfactory navigation by seabirds. Biology Letters 1:303-305.

Norris, K. S. 1967. Some observations on the migration and orientation of marine mammals. Pages 101-125 R. M. Storm ed.in Animal Orientation and Navigation. Corvallis: Oregon State University Press.

Norris, T. F., M. M. Donald and J. Barlow. 1999. Acoustic detections of singing humpback whales (Megaptera novaeangliae) in the eastern North Pacific during their northbound migration. The Journal of the Acoustical Society of America 106:506-514.

Papi, F., H. C. Liew, P. Luschi and E. H. Chan. 1995. Long-range migratory travel of a green turtle tracked by satellite: evidence for navigational ability in the open sea. Marine Biology 122:171-175.

Rossel, S. 1993. Navigation by bees using polarized skylight. Comparative Biochemistry and Physiology Part A: Physiology 104:695-708.

Sand, O. and H. E. Karlsen. 1986. Detection of infrasound by the Atlantic cod. Journal of Experimental Biology 125:197-204.

Simpson, S. D., M. Meekan, J. Montgomery, R. McCauley and A. Jeffs. 2005. Homeward sound. Science 308:221-221.

Stimpert, A. K., D. N. Wiley, W. W. L. Au, M. P. Johnson and R. Arsenault. 2007. "Megapclicks": acoustic click trains and buzzes produced during night-time foraging of humpback whales (Megaptera novaeangliae). Biology Letters 3:467-470. 
Walker, M. M., J. L. Kirschvink, G. Ahmed and A. E. Dizon. 1992. Evidence that fin whales respond to the geomagnetic field during migration. Journal of Experimental Biology 171:67-78.

Wallraff, H. G. 2004. Avian olfactory navigation: its empirical foundation and conceptual state. Animal Behaviour 67:189-204.

Wartzok, D. and D. R. Ketten. 1999. Marine Mammal Sensory Systems. J. E. Reynolds III and S. A. Rommel eds. in Biology of Marine Mammals. Smithsonian Institution.

Wiltschko, R. and W. Wiltschko. 2003. Avian navigation: from historical to modern concepts. Animal Behaviour 65:257-272. 


\section{Chapter 2}

Analysis of geomagnetic inclination and intensity as navigation cues in humpback whale migration tracks 


\subsection{Introduction}

The humpback whale (Megaptera novaeangliae) has the best studied migration of any whale species. These whales undertake one of the longest annual migrations of the baleen whales, with documented movements of up to 10,000 km Stevick et al. (2011). Populations of humpbacks exist in most oceans of the world, and all migrate between high latitude feeding areas and low latitude breeding and calving grounds (Stone et al. 1990, Stevick et al. 2004, Rasmussen et al. 2007). While some of these migrations occur over the continental slope, the majority of populations traverse wide stretches of open ocean during their annual trips (Norris 1967).

The migration of these whales is an energetically costly trip (Lockyer 1981, Kshatriya and Blake 1988). Although there is some anecdotal evidence of opportunistic feeding during travel and on the breeding grounds (Gendron and Urban 1993), it is generally thought that humpbacks fast for the entire roundtrip migration and breeding season. Therefore, it benefits the whales to make this trip as efficiently as possible, not only to save energy reserves, but also to maximize time on the breeding and feeding grounds. The fastest migration on record for a humpback is from an individual moving at least 4,400 km from southeastern Alaska to Hawaii in just 39 days (Gabriele et al. 1996). A recent study by Horton et al. (2011) has shown that migrating humpbacks are also capable of maintaining straight courses over distances of greater than $200 \mathrm{~km}$. This demonstrates that the whales are indeed capable of highly directed, rapid movement between their breeding and feeding grounds.

For the whales to successfully make these swift migrations, and save energy stores by avoiding unnecessary deviations, they must possess advanced navigational abilities. A miscalculation of just a few degrees at the start of a trip could lead an animal to miss its destination by hundreds of kilometers. Additionally, crossing the open ocean presents a particular challenge. The ocean is often thought of as a featureless environment, lacking any visual landmarks and devoid of stationary references that could provide traditional orientation cues. Migration in the ocean is further complicated by the presence of strong currents that can deflect an animal from its course. Yet, despite the 
widespread occurrence of these migrations, and the obvious navigational challenges that these long-distance movements pose, there have been almost no investigations into the actual sensory mechanisms whales use to traverse these great distances.

Contrary to the apparent uniformity of the open ocean, there are still numerous sources of navigation information available to a marine animal. The sun can be used as a compass, and both birds and sea turtles use this and other celestial cues to orient during their oceanic migrations (Wiltschko and Wiltschko 2003, Muheim et al. 2006, Lohmann et al. 2008b). Star patterns, the sun's position at sunset, patterns of skylight polarization, and the moon's position can also provide directional information (Wiltschko and Wiltschko 2003, Muheim et al. 2006, Lohmann et al. 2008b). Additionally, there are a wide range of physical features in the ocean that can provide useful navigation information. Despite the apparent homogeneity of the open ocean, many scales of structure in the physical environment can be used by an organism to locate and maintain position (Kobayashi et al. 2008). The North Pacific has shifting structures such as fronts, eddies and jets. Some fronts can be quasi-stationary and seasonally persistent (Belkin et al. 2009), providing a seasonal, stationary cue that migrating animals could utilize to aid in navigation. Other large-scale oceanographic indicators of direction include water masses or currents that might serve as signposts (Norris 1967), and sea surface temperature (SST), which could provide a reliable, albeit coarse resolution, indication of latitude. In addition to large-scale oceanographic features, there are smaller scale physical features that could aid in navigation. Waves are generally refracted as they pass around islands, creating characteristic interference patterns that could be detected by the accelerations and water movements associated with the waves (Lohmann et al. 2008b). In addition, water or airborne chemical cues may provide location information that an animal detects by taste or smell (Lohmann et al. 1999).

There are two methods of navigation available to a marine animal: vector and true navigation. Vector navigation is characterized by the ability of an animal to maintain a pre-determined compass bearing for a specific period of time or distance in order to reach its destination (Bingman and Cheng 2005). This can be done with a single sustained 
heading, or by a sequence of bearings to a series of goals. Vector navigation requires an animal to have a compass, which can be any cue that allows it to hold a steady heading. True navigation is the ability to navigate to a destination even in the event of deflection or displacement. True navigation requires both a compass and map. In this context, a map does not necessarily mean an exact representation of a geographic location. Instead, it encompasses all uses of positional information including the animal's general location in reference to other navigational cues or its destination. An animal using this method would first establish its position relative to the goal (map), and then determine the heading towards the destination (compass).

For an animal to navigate across the open ocean and maintain correct orientation in the face of deflecting currents, it must possess some form of true navigation. Navigation via a compass alone cannot detect deviations from an intended course or correct for them, and even small deflections from an animal's course can lead to large errors in vector navigation. While there are multiple cues that can be used as compasses, none of those mentioned so far can function as a map. Of all available orientation cues, the earth's magnetic field is the only ubiquitous cue that can provide both map and compass information (Lohmann et al, 2007). The geomagnetic field roughly resembles a giant bar magnet, with field lines that leave the planet in the southern hemisphere and enter the planet in the northern hemisphere (Figure 2.1). At each location on the globe, the magnetic field intersects the earth at a specific angle of inclination, which varies roughly with latitude (Figure 2.1 ). In addition, the intensity, or strength, of the magnetic field varies across the surface of the earth, but in a less predictable pattern than inclination. Intensity has three measurable parameters: the total intensity of the field, the horizontal component of intensity, and the vertical component of intensity (Figure 2.1). 

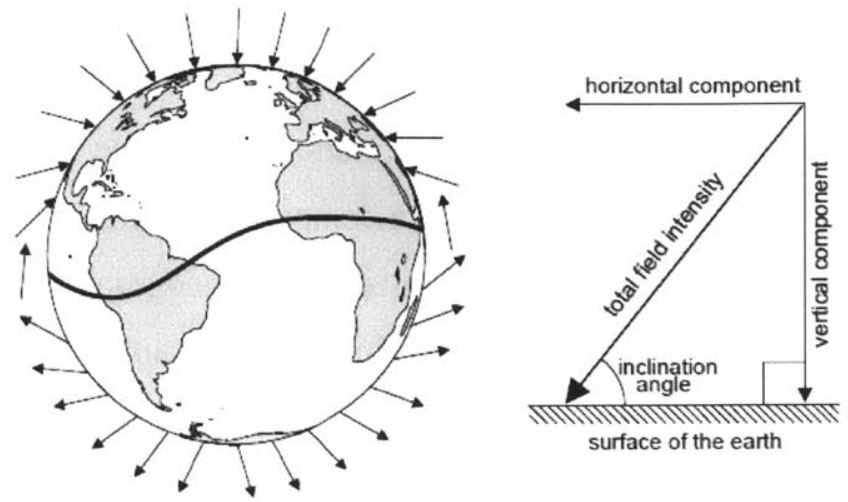

Figure 2.1: Left: A diagram of the angles that the geomagnetic field lines leave and enter the earth's surface. These angles change with latitude, with the field lines parallel to the earth's surface, an inclination angle of $0^{\circ}$, at the equator, and directed straight into or out of the earth at the magnetic poles (inclination of $\pm 90^{\circ}$ ).

Right: A diagram illustrating the different features of the geomagnetic field. The field at each location can be described in terms of total field intensity and inclination angle. The intensity can further be divided into its two vectors: horizontal intensity and vertical intensity. (Figure from: Lohmann et al. 1999)

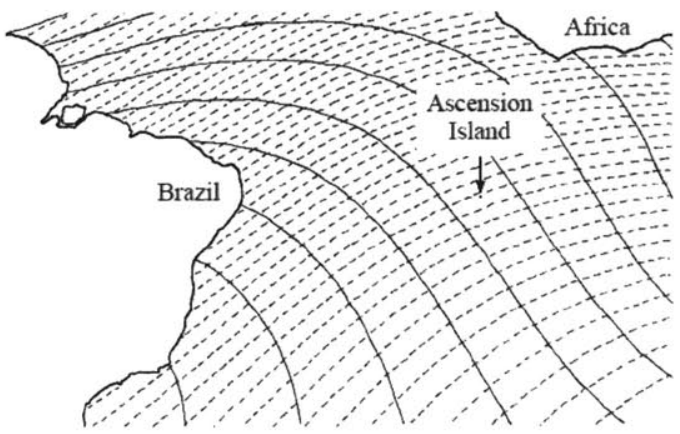

Figure 2.2: A map of the lines of equal inclination, isoclinics (dashed lines), and lines of equal intensity (isodynamics) in the South Atlantic. The two fields are non-orthogonal in this region, but the difference between the gradient field angles is still large, which would allow for more accurate navigation utilizing these fields. (Figure from: Lohmann and Lohmann 1996a)

Over the past two decades, evidence has accumulated that a variety of animals are capable of not only detecting the earth's magnetic field, but also of using it for navigation. 
Hatchling and juvenile sea turtles can detect both the inclination and intensity of the magnetic field (Light et al. 1993, Lohmann and Lohmann 1994, Lohmann and Lohmann 1996b, Lohmann et al. 2001, Luschi et al. 2007). Furthermore, these turtles have been shown to utilize both of these features as a 'magnetic map' to aid in navigation (Lohmann et al. 2004, Lohmann et al. 2011). Similar magnetic navigation has been found in a wide variety of taxa, including birds, bats, spiny lobsters, hamsters, mole rats, and even newts (Marhold et al. 1997, Fischer et al. 2001, Boles and Lohmann 2003, Deutschlander et al. 2003, Cochran et al. 2004, Holland et al. 2006). Several studies have suggested that cetaceans may also possess a geomagnetic sense. There is a correlation between live stranding events and geomagnetic minima or disturbances (Kirschvink et al. 1986, Klinowska 1986), as well as an association between sightings of migrating fin whales and geomagnetic minima (Walker et al. 1992). While there is, as yet, no concrete evidence that marine mammals possess a magnetic sense, the diversity of animals using the earth's magnetic field for a variety of navigational tasks indicates that a magnetic sense may be an essential component of successful true navigation.

While the importance of the earth's magnetic field in navigation has been demonstrated for many animals, the method with which they utilize magnetism to navigate is not well understood. Because inclination and intensity vary in a predictable way across the surface of the earth, the combination of these two factors can provide a 'magnetic map' (Figure 2.2). In this case, inclination and intensity would form a gradient map, which is a map based on at least two gradients that vary systematically over a region (Able 2001). A whale that is in an unfamiliar area could obtain its position relative to home by comparing the remembered values of each field at home with the values at its current location. Using this method, a whale could move in the direction, either up or down each field gradient, such that the current field value approaches the value at home. This means that the values of inclination and intensity are not being used as unique coordinates corresponding to a geographic location, but instead as an indication of general position relative to home. 
Traveling in this manner requires two gradients that vary in different directions. However, determining the correct direction to home would require a whale to take both fields into account at once. Benhamou (2003) suggests that such a calculation may be quite complex, and proposes a method by which an animal could instead arrive at an approximate solution by taking each field into account separately. However, unless the two fields are perfectly orthogonal, which is not the case anywhere on earth, using this method to navigate would generate systematic directional errors in the path of the animal. This bias would be greater the more acute the angle between the two fields. Such a directional bias has been noted in displacement experiments of green sea turtles (Benhamou et al. 2011). A regular error pattern in the track of any migrating animal could provide interesting information about the navigation process at work.

Most navigation studies to date have focused on small, easily manipulated animals, with methods focused on experimental displacement in the field as well as lab experiments. This approach is not possible with large cetaceans. However, with the advent of long-term satellite-monitored tags for large whales in the last several decades it is now possible to obtain precise data on the movements of whales on their breeding and feeding grounds, as well as along their migration routes. These observations, in combination with environmental and physical data, now allow us to look at the possible cues a whale may be using to navigate across large ocean distances.

By plotting the course a whale would take based on a specific cue or set of cues, we can compare the modeled course to the actual path taken by the whale. In this way, a correlation between the path taken when using certain cues, and the path the whale actually takes, can be established. In this study I utilize the satellite tracks of humpback whales migrating from Hawaii to Alaska, provided by Dr. Bruce Mate (Oregon State University), to test whether there is any correlation between available geomagnetic cues and the paths the animals followed. I utilize the theoretical method of navigation via two gradient fields proposed by Benhamou (2003) to model the course each tagged whale would take if it was only using magnetic inclination and intensity to navigate. By comparing this modeled track to the actual path taken by each whale, I test the hypothesis 
that humpback whales are capable of sensing the earth's magnetic field and using it to navigate. The systematic direction bias expected by the method proposed by Benhamou (2003) provides a noticeable deviation from the most direct route to a destination, and this can be compared to deviations in the observed whale tracks. It is generally assumed that migrating animals utilize multiple navigational mechanisms, with different scales of accuracy, over the course of their migration (Bingman and Cheng 2005). Because the whales are likely using all the navigation information available to them, an exact match between the modeled and actual whale paths is not expected, as the model only utilizes the navigation information available from the earth's magnetic field.

\subsection{Methods}

\subsubsection{Satellite track collection}

The satellite tracks in this study are from humpback whales tagged in Hawaii between March 1995 and February 2000. Dr. Bruce Mate conducted the tagging study, in an Oregon State University project, with location-only, Argos, satellite-monitored, ultra-high-frequency radio tags, and he provided the data for this project. The Argos data collection and location system uses tag transmissions collected by receivers onboard NOAA TIROS-N series weather satellites, which are in a sun-synchronous polar orbit. The locations are calculated from the Doppler shift in the frequency of the transmitted signal as the satellite moves overhead (Fancy et al. 1988). Location accuracy depends on the number of received messages, with a minimum of two messages required to obtain a location.

Two different types of tags were deployed during this time period, with attachment improvements made to the second version. In 1995, the tags consisted of Telonics ST-10 transmitters housed in a stainless steel tube with two stainless steel subdermal anchors silver-soldered to the housing (Figure 1 in Mate et al. 1998). Each anchor had a cutting blade and 10 stainless steel wires attached to the distal end. An antenna and a salt-water switch, which was used to prevent the tag from transmitting underwater, were mounted in the endcap. The remaining tags consisted of Telonics ST- 
15 transmitters housed in $19 \mathrm{~cm}$ long fully implantable stainless steel tubes (Mate et al. 2007 Figure $3 b$ ) . Four double-edged blades were mounted in a tapered endcap, with a flexible whip antenna and salt-water switch mounted at the other end. All tag transmissions were set on a duty cycle in order to conserve power.

The whales were tagged in the waters surrounding the islands of Kauai and Maui in Hawaii. The tags were deployed from small $(5-7 \mathrm{~m})$ rigged-hull inflatable boats, utilizing a modified Barnett compound crossbow. Groups of fewer than six animals were slowly approached to within 1-7 m for tagging. Placement was ideally located 0.3 to $1 \mathrm{~m}$ forward of the dorsal fin or hump, and within $20 \mathrm{~cm}$ of the mid-dorsum. Ideally, the anchors penetrated the muscle of the animal, which enhances the duration of the tags.

\subsubsection{Location filtering and plotting}

Argos locations are classed based on the number of messages received and their spacing during satellite passage over the transmitter. At least four successive uplinks during a satellite pass are necessary for a location to be assigned an estimated error (Argos 2011). The error is estimated as a radius, but in most cases there is significantly more error in longitude than in latitude, making the error more accurately approximated by an ellipse (Argos 2011). All class $Z$ locations were removed from the data, as this class indicates that the location process failed. Location classes 0 to 3 correspond to an error of approximately 250 to $1500 \mathrm{~m}$ (Argos 2011). The last two location classes, A and $B$, are not assigned an error estimate due to the reduced number of received messages ( 3 messages for location class A, and 1 or 2 messages for location class B). However, a study by Vincent et al. (2002) estimates that location class A and B still provide acceptable accuracy, especially when the range of movement of the animal is large. Therefore, as this is not a fine scale study of movement, quality should not make a large difference in the results and all remaining location classes were utilized. These locations

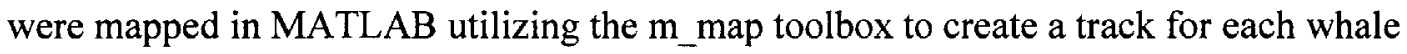
(R. Pawlowicz, UBC). 
This study concentrates on the movements of each whale during migration only. In order to focus on this transit section, each track was visually assessed for the location from which the whale was judged to have left the Hawaiian Islands, and this location was then utilized as the starting point for migration. This process removed any inter-island movement, during which the animals may have been breeding. The end of each migration track was either defined as the last transmitted location, or visually assessed as the point at which the animal appeared to reach its destination and began movement indicative of foraging. Because there is no definitive location for 'home' in any of these tracks, and several of the tags stopped transmitting before the whale reached any destination, the chosen home location is subject to some interpretation and may bias the results.

Once the transit section of each track was defined, a great circle route was plotted between the start and end points of the transit. A great circle route is the shortest path between two points on a sphere and thus represents the shortest route a whale could take. A rhumb line, the constant heading course between two points, was also plotted on the map. In each of these cases, the distances were such that the rhumb line and great circle courses were relatively close together. In almost all cases, the whale's course does not closely match either the great circle or rhumb line course, and in many cases it actually deviates systematically to one side of the great circle route. These systematic deviations led to consideration of what factors could lead a whale on a regular divergence from the most direct course. These deviations are similar to the deviation pattern predicted by Benhamou (2003) for an animal navigating via a bicoordinate system based on nonorthogonal fields. Therefore, these divergences can be used to determine the role of geomagnetic field parameters in humpback whale navigation.

\subsubsection{Modeling of bicoordinate geomagnetic navigation track}

The methods of Benhamou (2003) can be utilized to model navigation for any two gradient fields. The earth's magnetic field is the most prevalent of the available navigation cues, and is generally agreed to be the most likely candidate for a 'map' sense 
in animals (Wiltschko and Wiltschko 2005, Lohmann et al. 2007). Because of these factors, I chose to use the inclination and intensity of the geomagnetic field as the two gradient fields with which to model a course according to Benhamou (2003). In order to implement this model, the values for inclination and total intensity were obtained, at 0.5 arc degree intervals, for the range of the tracks in the North Pacific. These field parameters were calculated using the 11 th generation of the International Geomagnetic Reference Field (IGRF-11) in the MATLAB Aerospace Toolbox (Finlay et al. 2010). The isoclinics (lines of equal inclination) and isodynamics (lines of equal intensity) were then mapped with each of the whale tracks.

The model was initiated at the start location for the transit phase of each track. Using the methods of Benhamou (2003), a heading was calculated using the field parameters for inclination (field $A$ ) and intensity (field $B$ ). According to this method, an animal computes the movement to perform for each field separately, in the direction of each gradient, such that the current field value becomes equal to the home field value. The home heading is then estimated as the direction $\theta$ from the vector sum $\vec{V}_{A}+\vec{V}_{B}$. The distances of these vectors are equal to:

$$
D_{A}=\frac{\left(A_{H}-A_{C}\right)}{A^{\prime}}
$$

and

$$
D_{B}=\frac{\left(B_{H}-B_{C}\right)}{B^{\prime}}
$$

where $A^{\prime}$ and $B^{\prime}$ are the gradient strengths (steepness) of each field, $A_{H}$ and $B_{H}$ are the values of each field at the home location, and $A_{C}$ and $B_{C}$ are the field values at the current location.

The Cartesian coordinates for these distances, with the $X$-axis set to a compass direction of due east, are:

$$
\begin{aligned}
& X_{A}=D_{A} \cos (\alpha) \\
& Y_{A}=D_{A} \sin (\alpha)
\end{aligned}
$$

and

$$
X_{B}=D_{B} \cos (\beta)
$$




$$
Y_{B}=D_{B} \sin (\beta)
$$

where $\alpha$ and $\beta$ are the directions of the gradients of each field as measured counterclockwise from the east.

The predicted heading the animal should take according to the model is then calculated as:

$$
\theta=\arctan \left(\frac{Y_{A}+Y_{B}}{X_{A}+X_{B}}\right)
$$

The heading was calculated in this manner at the start of the track, and then the track was advanced $5 \mathrm{~km}$ and the heading was recalculated using the field values at that location. Five kilometers was chosen as a sampling distance that was sufficiently fine to demonstrate all the features of the model, without requiring exceptional computation time. This was repeated until the track reached the home location. The resultant model track was then plotted on a map of isoclinics and isodynamics, with the path the whale actually followed, and the great circle route between the start and home. The deviation direction of each observed whale track from the great circle route was then compared to the deviation direction of the model.

The assumption behind the above 'simple' method is that an animal is incapable of taking both fields into account at once, a calculation that would allow for more direct navigation. In order to test whether or not this is the case, the method for 'true' navigation according to a bicoordinate system was modeled as well. This model was initiated in the same manner as above, utilizing the start and end points for each track. This model defines the heading to home $(\phi)$ as the vector sum $\vec{U}_{A / B}+\vec{U}_{B / A}$ where $\vec{U}_{A / B}$ is a vector orthogonal to field $B$, which approaches the destination value for field $A$. The length of $\vec{U}_{A / B}$ is equal to $L_{A / B}=\left|D_{A} / \sin (\Delta)\right|$ where $\Delta$ is the angle between the two field gradient directions and is between $0^{\circ}$ and $90^{\circ} . \vec{U}_{B / A}$ is a vector orthogonal to field $A$ that approaches the destination value for field $B$, with length $L_{B / A}=\left|D_{B} / \sin (\Delta)\right|$. The Cartesian coordinates for these vectors are: 


$$
\begin{aligned}
& X_{A / B}=\frac{D_{A} \cos \left(\beta-90^{\circ}\right)}{\sin (\beta-\alpha)} \\
& Y_{A / B}=\frac{D_{A} \sin \left(\beta-90^{\circ}\right)}{\sin (\beta-\alpha)}
\end{aligned}
$$

and

$$
\begin{aligned}
& X_{B / A}=\frac{D_{B} \cos \left(\alpha-90^{\circ}\right)}{\sin (\alpha-\beta)} \\
& Y_{B / A}=\frac{D_{B} \sin \left(\alpha-90^{\circ}\right)}{\sin (\alpha-\beta)}
\end{aligned}
$$

The heading is then calculated as:

$$
\phi=\arctan \left(\frac{Y_{A / B}+Y_{B / A}}{X_{A / B}+X_{B / A}}\right)
$$

The heading was calculated at the start of the track, and then the track was advanced 5 $\mathrm{km}$ and the heading recalculated using the field values at that location. This was repeated until the track reached the home location. The resultant track was then compared to the path the actual whale took and the direction of the deviation from the great circle route.

\subsubsection{Binomial test}

A binomial test was used to determine if the number of tracks where the observed deviation matches the predicted model deviation was greater than would be expected by chance. Each whale track was categorized according to the direction that it deviated from the great circle route. Because the tracks are roughly oriented north-south, the deviations can be categorized as either to the west or east of the great circle route. The direction of the simple and 'true' bicoordinate model were then categorized in this way, compared to the actual deviation, and determined to be a match or not (Table 2.2). The track for Whale 1 was excluded from this section of the analysis, as it is the shortest track and follows the great circle route quite closely. This gives a total sample size of nine whales for the binomial test. The number of whales for which each model deviation direction matched the observed deviation direction was summed. This total, along with the sample size, was utilized to conduct a one-tailed binomial test of whether the observed number of 
matching deviation directions are significantly greater than would be expected if there was an equal chance of the modeled track falling on the same side as the observed track. The power of this test was then calculated, along with the sample size required to obtain a binomial test with a power of 0.8 .

\subsubsection{Testing of model calculations utilizing simulated field parameters}

When utilizing a theoretical model, such as that presented in Benhamou (2003), with real-world field parameters, it is difficult to discern if any deviations from the expected model are due to errors in the calculation or to variations in the fields used. The field parameters utilized here, magnetic inclination and intensity, vary non-uniformly across the earth's surface, with both the field direction and the gradient steepness changing with location. In addition, the whales are navigating on the surface of a spherelike geoid. This means that the shortest distance between two points is not a straight line, but is instead a curve. Both of these factors mean that a route calculated between two points on the earth according to the methods of Benhamou (2003), may vary significantly from the route predicted in the flat, uniform model. In order to ensure that the differences between the theoretical and real-world models were due to these differences, rather than to errors in the calculations themselves, I calculated bicoordinate navigation paths in a simplified system with uniform fields on a flat surface.

In MATLAB, two fields ( $A$ and $B$ ) were initialized, with uniform steepness and direction. The values for each field were established at $5 \mathrm{~km}$ intervals on a flat plane $\left(1^{\circ}\right.$ latitude $=1^{\circ}$ longitude). Once the field values were established at each location, start and home locations were arbitrarily chosen and a straight-line path drawn between the two. The bicoordinate route was then modeled according to the parameters of the two fields. Using the values for each field, the directions and steepness of A and B were calculated at each grid location according to the same methods employed for the real-world model. The field values were interpolated for the start and home locations, and using these values, and the field directions and gradients at the start location, a heading towards the home location was calculated. The track was advanced by $5 \mathrm{~km}$, the field parameters at 
the new location established, and a new heading determined. In this way, the track was advanced until it reached home. This iteration was repeated for a wide variety of field directions, gradient steepnesses, and start and end locations. These same methods were then repeated for the complex method of bicoordinate navigation, taking both fields into account at once.

\subsubsection{Accounting for surface ocean currents}

The systematic biases seen in many of the migration tracks could be due to many different factors. This study tests whether or not the deviations are due to a direction bias based on navigation via specific cues. However, the whales are also subject to the influence of ocean currents encountered as they migrate. The observed ARGOS tracks are the whales' ground-related movements, which are a combination of the locomotor movement of each whale within the water mass as well as the movement of the water itself. Therefore, it is not immediately apparent whether any deviations in the observed track are due to intentional movement by the whales, or are the result of deflecting currents. In order to get an accurate estimation of the whales' actual navigation efficiency, the influence of any currents must be removed, and each whales' motor movements estimated.

Humpback whales are not deep divers, spending most of their time at depths of less than $60 \mathrm{~m}$ during foraging (Dolphin 1987). Therefore, it is assumed here that the main influence on their movements will be due to surface currents. Geostrophic surface current vectors were obtained from the Ssalto/Duacs maps of absolute dynamic topography, available weekly on a $1 / 3^{\circ}$ grid (www.jason.oceanobs.com). Geostrophic currents are a result of the balance between the horizontal pressure gradient force and the Coriolis force, and this is expected to be representative of the near surface currents in this region. The east $(\mathrm{U})$ and north $(\mathrm{V})$ geostrophic current vectors at each whale location and time were estimated utilizing the weekly current maps.

The $\mathrm{x}$ and $\mathrm{y}$ vectors for each whale location were calculated using the speed and heading of the whale between successive points. The oceanic current vectors were then 
subtracted from the whale ground vectors at each location. This gives the motor vectors for each location $(\vec{x}, \vec{y})$, from which a current-corrected heading $(\theta)$ was then calculated according to $\theta=\arctan \left(\frac{\vec{y}}{\vec{x}}\right)$. These headings, and the distances between locations, were used to plot each whale's actual swimming path. This swimming path was then compared to the whale's ground related path.

\subsubsection{Test for bearing correction}

The simple modeled track from above takes the start location and the home location for each whale and plots a course between the two, updating the magnetic field parameters every $5 \mathrm{~km}$ along the track. While this method approximates the track a whale would take if it were solely following the magnetic inclination and intensity from start to finish, each whale navigates more accurately than is predicted by this model. This improved accuracy could be due to two things: utilization of other navigation cues, or maintenance of a set bearing correction. Many reviews of animal orientation predict that animals use multiple cues to navigate (Avens and Lohmann 2003, Bingman and Cheng 2005, Lohmann et al. 2008a), and the whales in this study could use these other cues to recognize and correct for some of the deviation introduced by the geomagnetic navigation, and thus improve the accuracy of their course. Additionally, the whales have all made at least one prior migration between the breeding and feeding grounds. Since they have traveled this route before, they may be aware of the extreme deviation caused by following a geomagnetic bicoordinate course, and thus may be capable of partially correcting for it. If the bicoordinate route consistently leads them to one side of their destination, the whales could adjust their heading by adding or subtracting a set bearing to the geomagnetic heading, and therefore arrive at a more accurate heading to home. In order to test for this possibility, I looked at the modeled bicoordinate heading compared to the actual heading taken by the whale.

At each whale location, a heading to home was calculated according to the simple bicoordinate navigation methods outlined above, utilizing the magnetic inclination and intensity field parameters at that location. The whale's observed heading was then 
calculated between the chosen location and the next observed location. The difference between these two headings was then calculated using a circular statistics toolbox for MATLAB (Berens 2009). The circular mean and standard deviation of these heading differences were calculated for each whale. If each whale employs a set bearing correction to the model bearing, then the standard deviation of the heading differences will be close to zero.

\subsection{Results}

\subsubsection{Observed tracks}

Of all tracks collected by Bruce Mate from 1995 to 2000, 10 were of sufficient length and quality to be considered for this study. These tracks ranged in length from about $1,000 \mathrm{~km}$ long to nearly $5,000 \mathrm{~km}$ long, and in duration from 8 days to 76 days (Table 2.1). In all cases, the straight-line distance to the chosen destination was shorter than the distance the whale actually traveled (Table 2.1).

Three of the 10 tracks made it all the way to the feeding grounds (Whales $4,7,8$ ), either in British Columbia or southwest Alaska (Figure 2.3). However, only one of these full-length tracks contains a large number of locations (Whale 8, Figure 2.11). Additionally, three of the tracks head towards, or arrive at, the Kermit-Roosevelt seamount range (Whales 8,9 and 10). Whale 8 spends over a month at this location before it changes its direction and continues on to southwest Alaska. There is a set of

ridges and seamounts called the Musicians Seamounts Trail to the north of the Hawaiian Islands, and seven of the whale tracks pass through this range at the start of their migration, regardless of the feeding destination to which they are heading (Figure 2.3). 
Table 2.1: A summary of the tagging and track information for each whale used in this study. The track for Whale 8 is split into two parts, $8 \mathrm{a}$ and $8 \mathrm{~b}$, with the two tracks split between before and after the stopover at the Kermit-Roosevelt Seamount.

\begin{tabular}{|c|c|c|c|c|c|c|c|c|}
\hline Whale & age class & Tag start time & Tag end time & $\begin{array}{l}\text { Total transit } \\
\text { time (days) }\end{array}$ & $\begin{array}{l}\text { Total \# } \\
\text { locations }\end{array}$ & $\begin{array}{l}\# \text { transit } \\
\text { locations }\end{array}$ & \begin{tabular}{|l} 
total distance \\
traveled $(\mathrm{km})$
\end{tabular} & $\begin{array}{l}\text { great circle } \\
\text { distance }(\mathrm{km})\end{array}$ \\
\hline 1 & adult & $3 / 27 / 1995$ 19:28 & 4/11/1995 11:56 & 8.85 & 32 & 25 & 1066 & 835 \\
\hline 2 & subadult & 4/2/1995 19:54 & 4/19/1995 21:05 & 13.61 & 58 & 39 & 1331 & 1219 \\
\hline 3 & adult female & 4/9/19970:18 & 5/21/1997 17:41 & 18.28 & 48 & 19 & 1479 & 1296 \\
\hline 4 & adult & 4/5/1997 18:15 & $6 / 23 / 1997$ 17:09 & 30.19 & 45 & 13 & 3681 & 3461 \\
\hline 5 & adult male? & 4/14/1998 21:40 & 5/23/1998 5:37 & 21.48 & 62 & 57 & 2832 & 2627 \\
\hline 6 & adult & 4/8/1998 21:24 & $5 / 4 / 199818: 37$ & 21.73 & 38 & 30 & 1988 & 1646 \\
\hline 7 & adult & $4 / 15 / 199819: 23$ & $8 / 10 / 199818: 16$ & 32.83 & 248 & 24 & 4319 & 4120 \\
\hline $8 a$ & adult & 2/11/1999 0:24 & $7 / 3 / 1999$ 14:34 & 22.64 & 468 & 65 & 2524 & 2036 \\
\hline $8 b$ & & & & 16.16 & & 47 & 2018 & 1744 \\
\hline 9 & adult & $2 / 10 / 199920: 31$ & 4/17/1999 5:56 & 22.01 & 94 & 26 & 2439 & 2253 \\
\hline 10 & adult & $2 / 5 / 20001: 46$ & $3 / 25 / 200018: 00$ & 25.54 & 112 & 56 & 2423 & 2011 \\
\hline
\end{tabular}




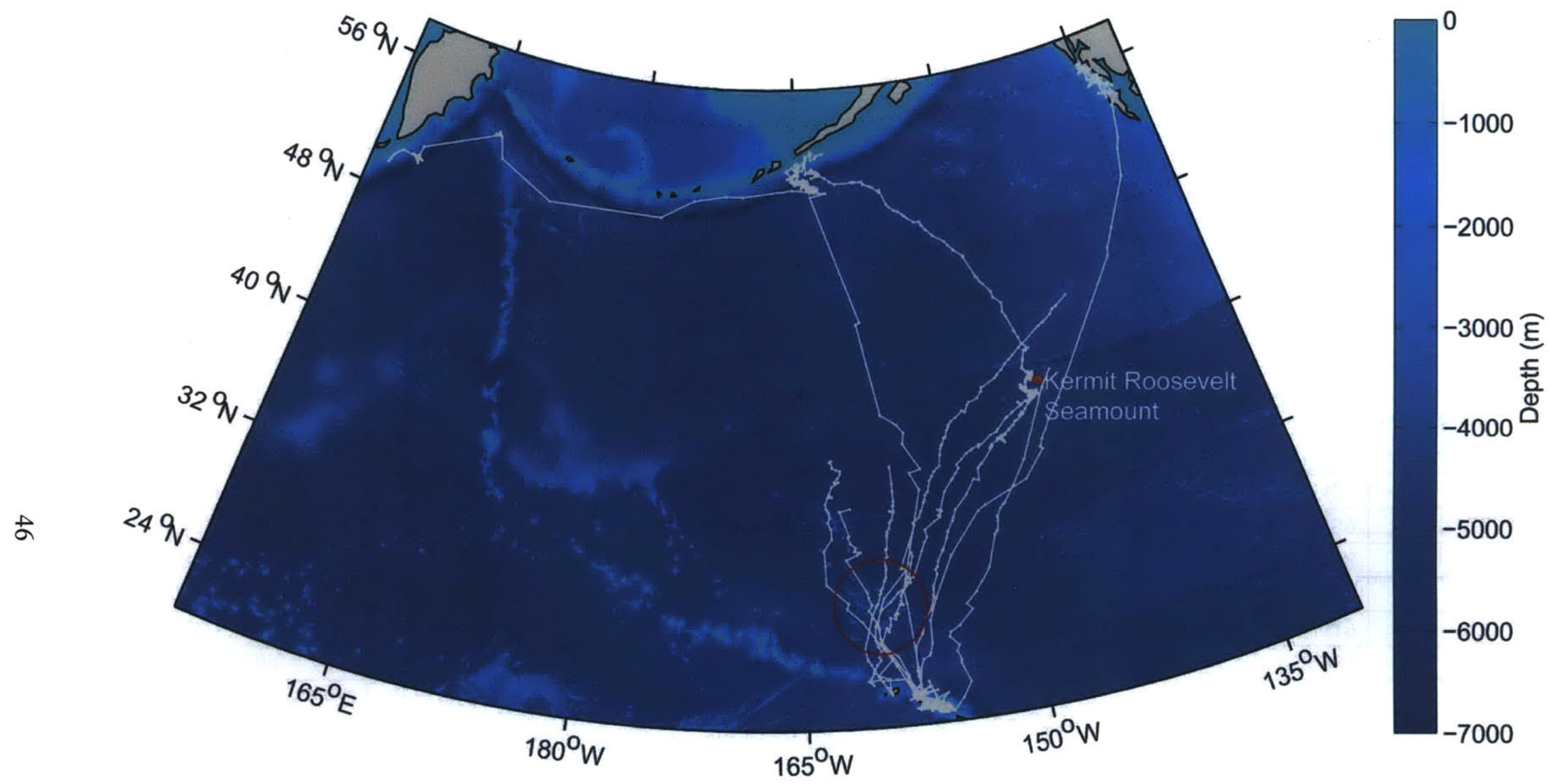

Figure 2.3: A 10 arc-minute bathymetric map of the study region with all 10 whale tracks plotted. The location of the KermitRoosevelt Seamount is marked in red, as well as the Musicians Seamount Trail (red circle). Bathymetric data from Amante and Eakins (2009). 
In order to define the transit section of each track, a start and end location was chosen. In most cases, the start location was simply the first location that the whale appeared to move away from the Hawaiian Islands. For Whale 2, the start of the track takes a course to the northwest, and then makes a turn towards the northeast. For this reason, the turning point was chosen as the start of the track (Figure 2.5). In the case of Whale 5, there is a gap between the last received location at the Hawaiian Islands, and the first obvious transit location. Because there is no way of knowing if the whale was moving among the islands during this time, the first transit location is chosen as the start (Figure 2.8). Whale 10 also exhibits a very straight course for the first few locations moving away from the islands, and then undertakes an abrupt change in heading; this change point was chosen as the start location (Figure 2.13).

For seven of the ten whales, the satellite tag did not last until the whale reached its destination. In these cases, 'home' was chosen as the last transmitted location, as there is no way to determine which feeding destination toward which each whale is headed. However, Whale 3 has a sudden shift in latitude between the last two locations; because the second to last location maintains the track the whale was on for the previous few locations it was chosen as the home location (Figure 2.6). For Whale 4, which reaches its feeding grounds in the Aleutian Islands, the home location is defined as the last location before the whale starts moving almost due west (Figure 2.7). The whale does not appear to stop and begin a search pattern indicative of feeding at this point, but once it reaches the Aleutian Islands it has likely switched to regional navigation cues, such as bathymetry. Whale 7 also reaches its destination in British Columbia, and in this case, makes a very obvious transition from migrating to prey search/foraging, and the location of landfall is chosen as home (Figure 2.10). 


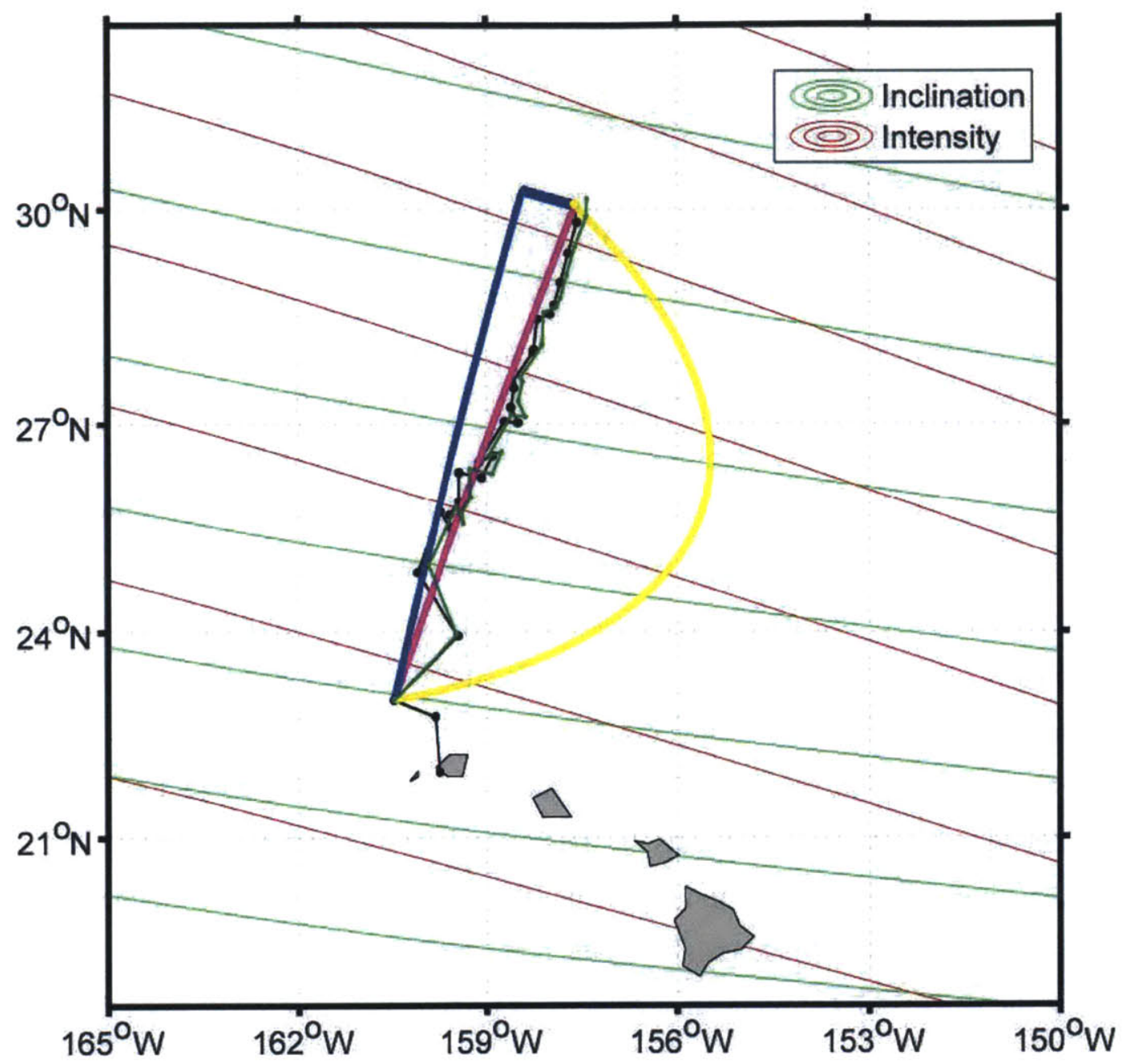

Figure 2.4: The track for Whale 1 plotted on a Mercator projection map. The isolines for inclination and intensity are marked in green and red. The observed whale track is in black, with the great circle route between the start and home locations marked in magenta. The simple bicoordinate route is marked in blue and the complex bicoordinate route is in yellow. The current-corrected track is plotted in dark green. This is the shortest track used for this study, just over $1,000 \mathrm{~km}$ long, and it follows the great circle route the most closely of any of the tracks. It is seen here that the complex bicoordinate route, which should follow the great circle route closely, actually deviates from the observed track the farthest. 


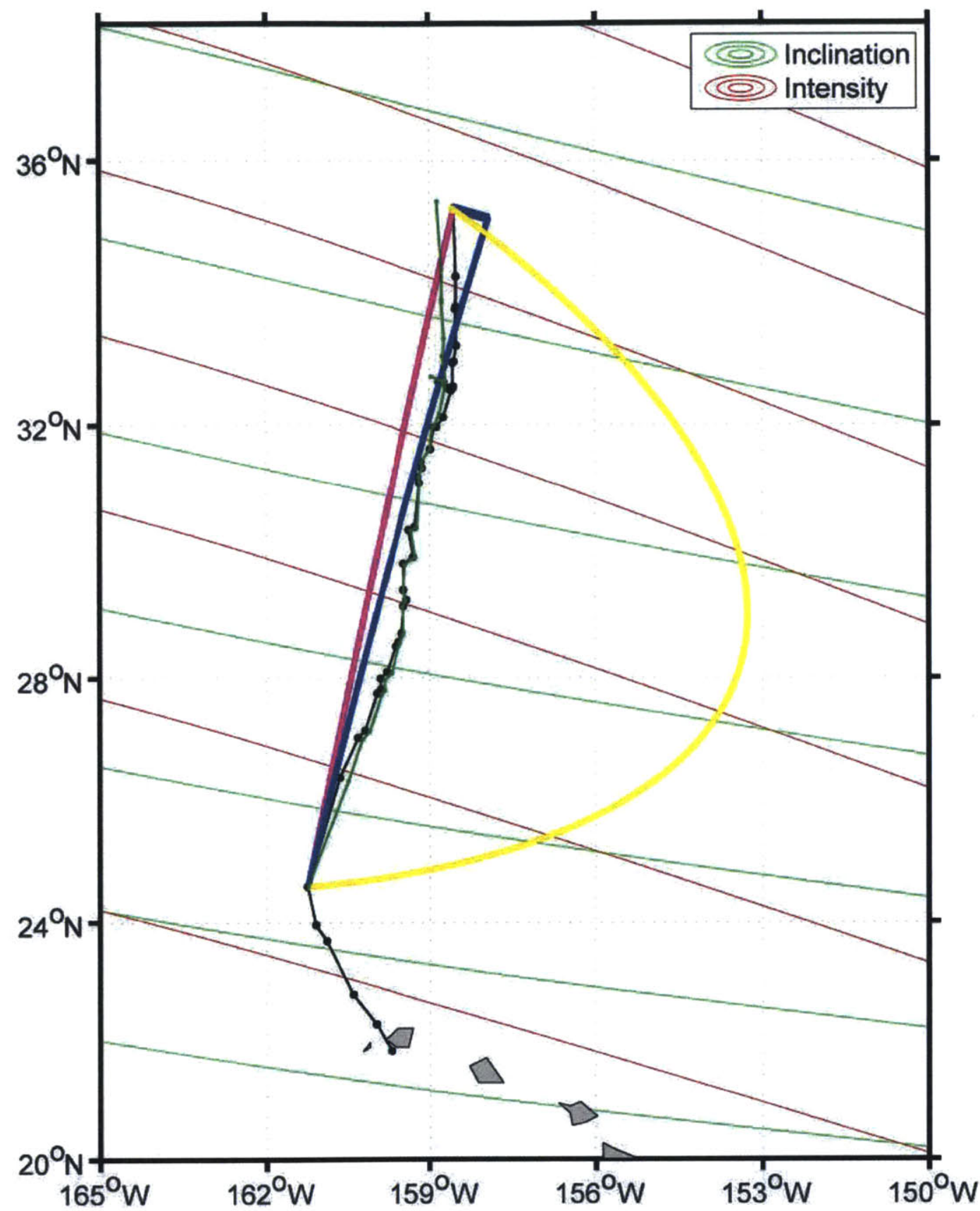

Figure 2.5: The track for Whale 2 plotted on a Mercator projection map. The color scheme is the same as in Figure 2.4. In this track, the observed track deviates in the same direction as the modeled bicoordinate track. The magnitude of both deviations is relatively small. The current-corrected track closely matches the observed track. The complex bicoordinate track deviates greatly from the predicted straight-line course. 


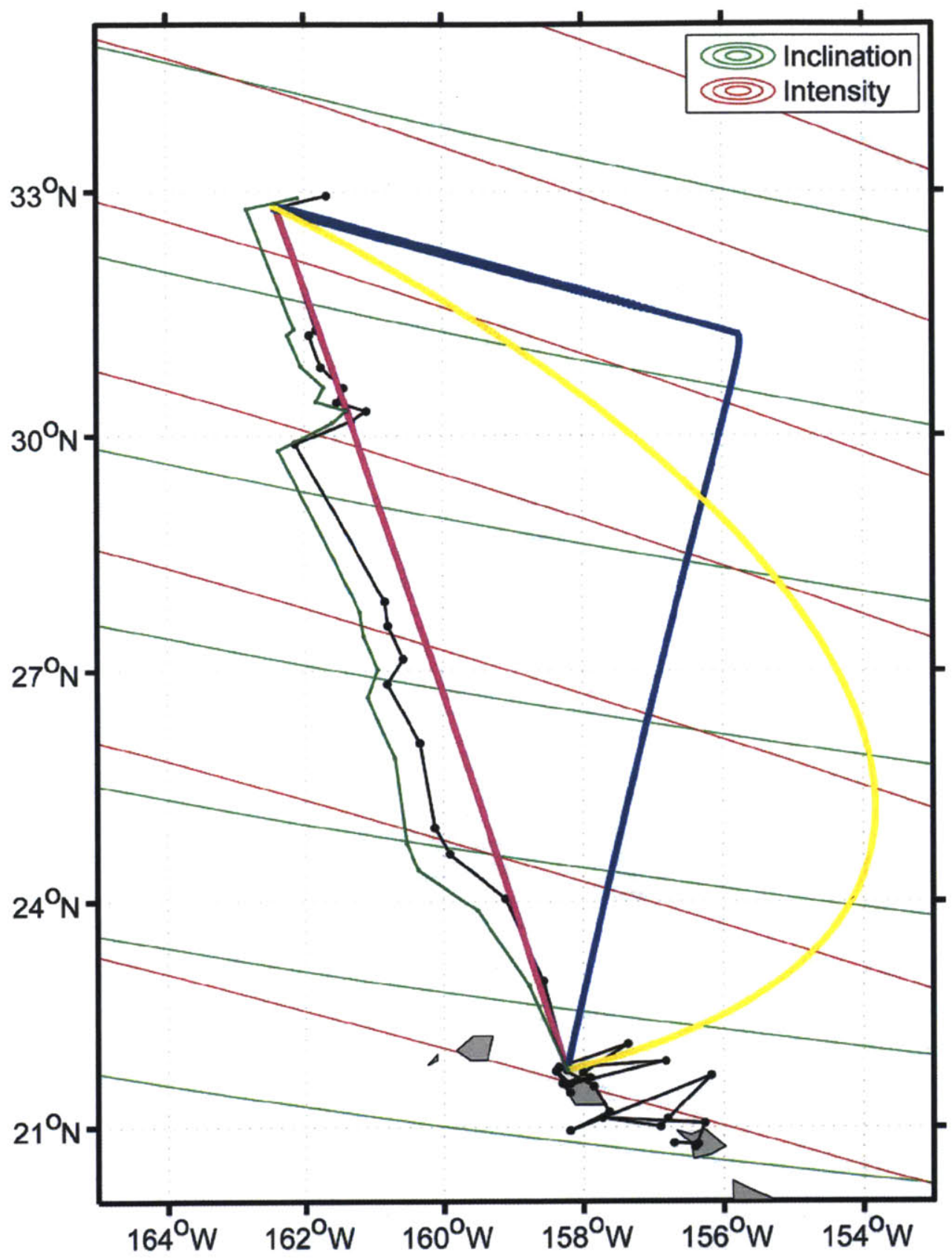

Figure 2.6: The track for Whale 3 plotted on a Mercator projection map. The color scheme is the same as in Figure 2.4. This track has relatively few locations (20 transit). It is one of only two tracks for which the observed route deviates in the opposite direction than is predicted by the bicoordinate model. In this track, the end location was chosen as the second to last point, due to the fact that this point is more in keeping with the previous few locations. 


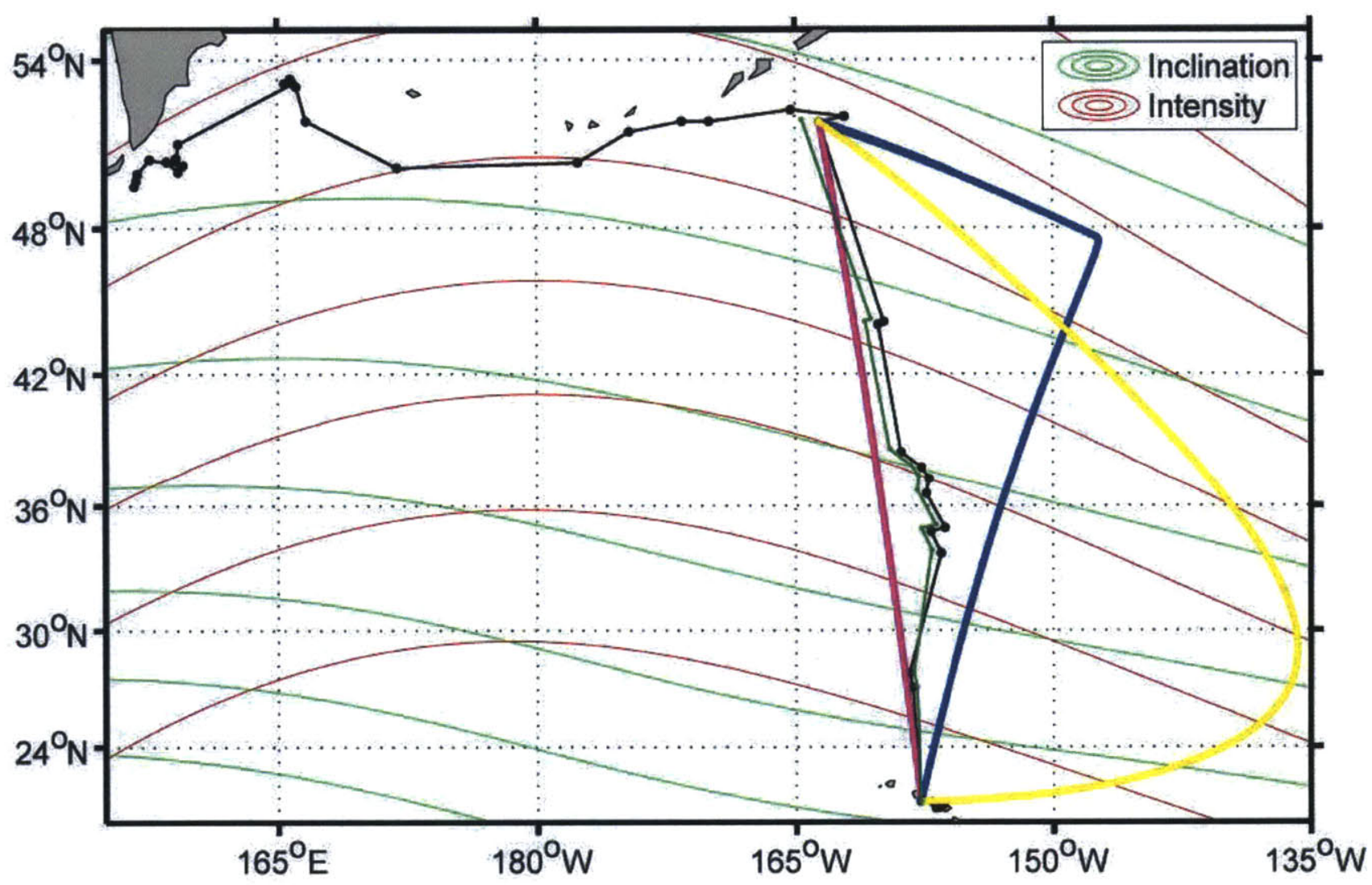

Figure 2.7: The track for Whale 4 plotted on a Mercator projection map. The color scheme is the same as in Figure 2.4. This is one of three tracks to make it all the way to the destination. This whale does not stop at the feeding grounds near the Aleutian Islands, but instead continues on to the Russian Kamchatka Peninsula. The tag stops transmitting before the whale ever appears to initiate foraging activity. The number of transit locations is relatively low in this track (13 locations), with the first few locations following the great circle route. The deviation of both the simple and true modeled track follows the same direction from the great circle route as the observed track. 


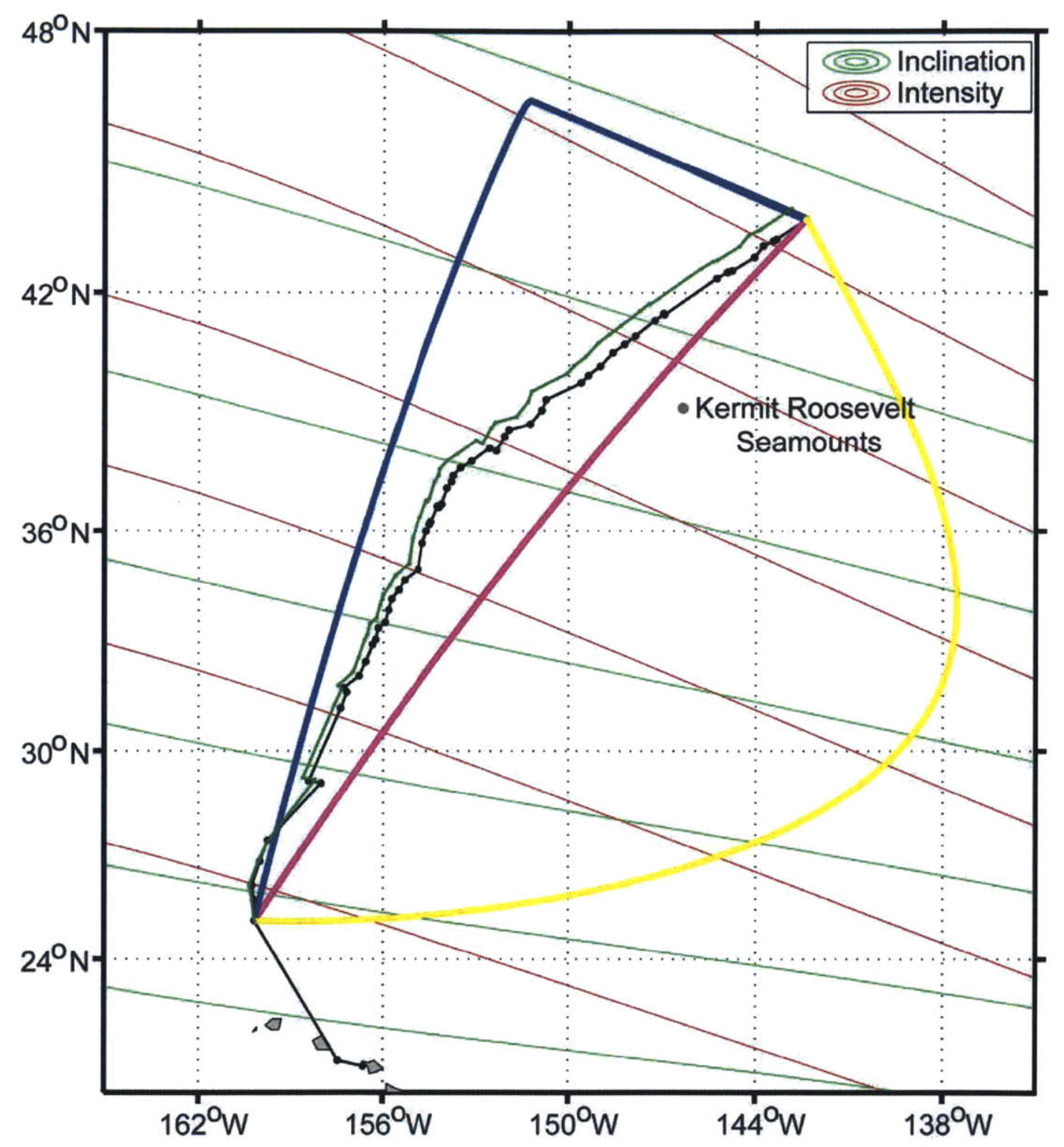

Figure 2.8: The track for Whale 5 plotted on a Mercator projection map. The color scheme is the same as in Figure 2.4. The observed track has a very systematic deviation away from the great circle route in the same direction as the modeled simple bicoordinate route. This whale goes near, yet completely bypasses, the Kermit Roosevelt Seamount. 


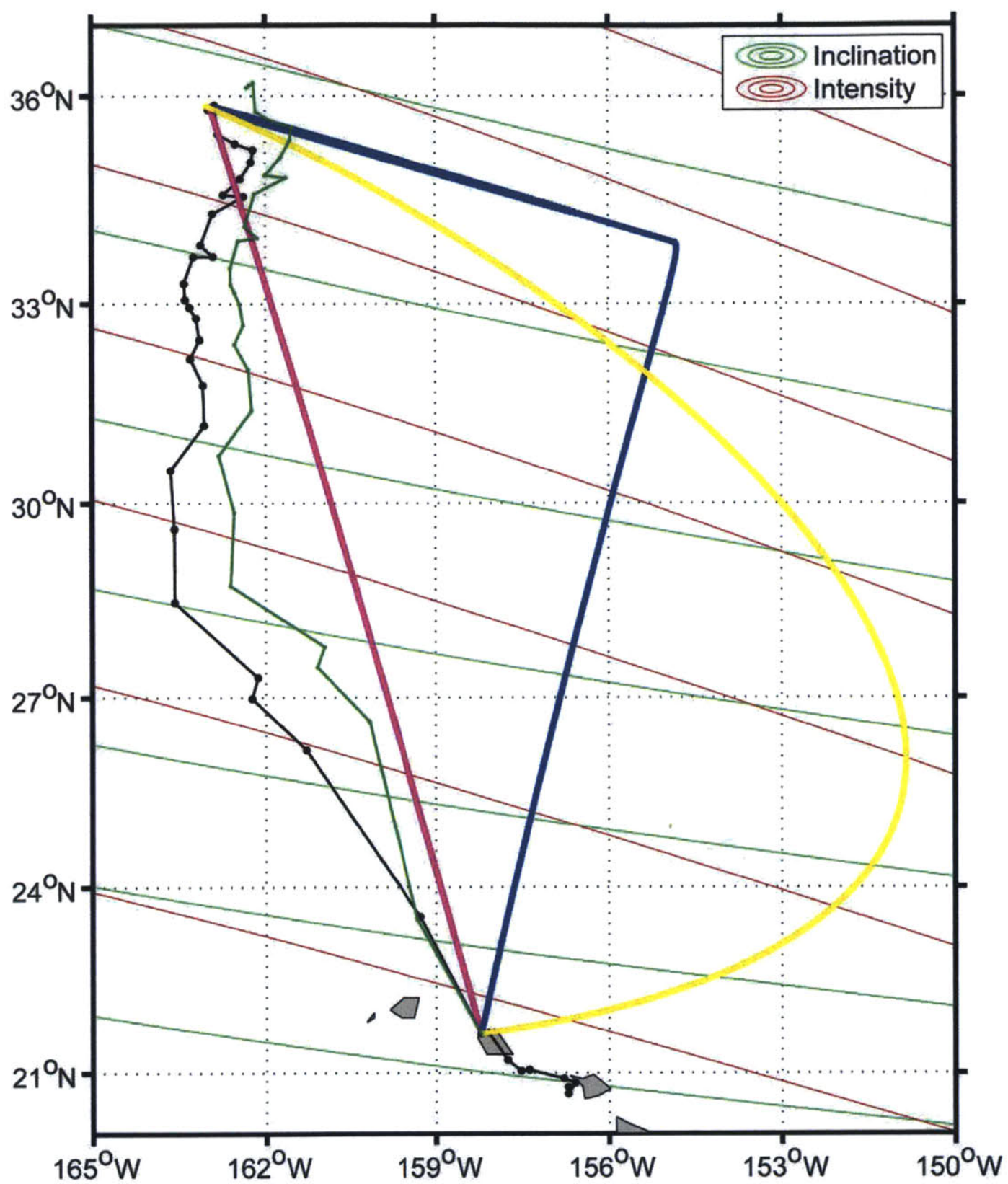

Figure 2.9: The track for Whale 6 plotted on a Mercator projection map. The color scheme is the same as in Figure 2.4. This whale is one of two that does not follow the deviation direction predicted by the bicoordinate model. The current-corrected track differs from the observed track more than that of the other whales. 


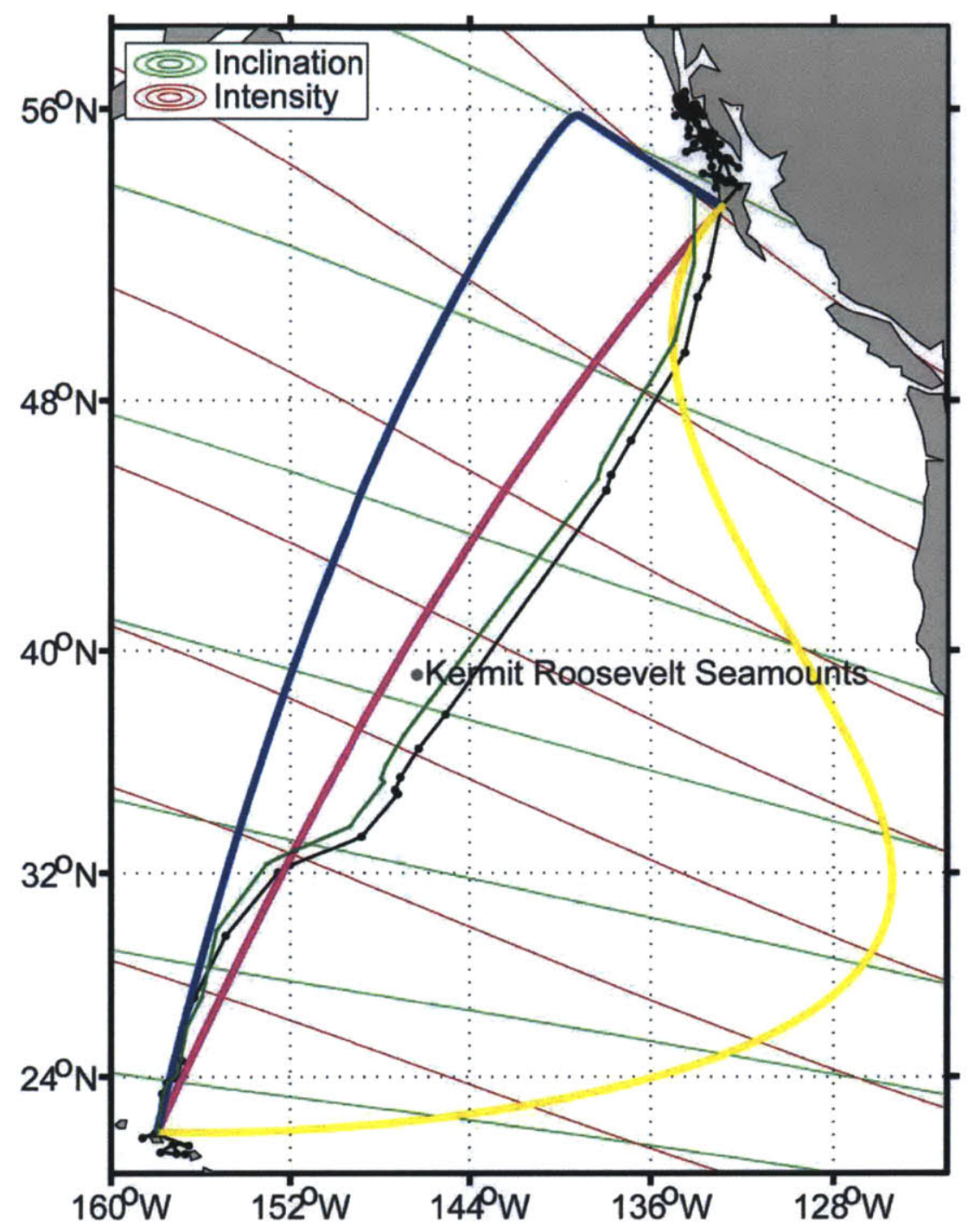

Figure 2.10: The track for Whale 7 plotted on a Mercator projection map. The color scheme is the same as in Figure 2.4. This whale track follows a different pattern than most of the other tracks. The initial track deviation matches the deviation direction predicted by the bicoordinate model. However, the whale soon switches to a course to the opposite side from that predicted by the bicoordinate model. There are relatively few transit locations in this track, and therefore it is difficult to determine precise points of deflection. There are a substantial number of locations once the whale reaches British Columbia, with movement patterns indicative of foraging. In this case, the modeled complex bicoordinate route makes a very large deviation to the east of the route, and then curves back around to the destination. This change in the pattern is likely due to the fact that the field directions for geomagnetic inclination and intensity change substantially across this region. 


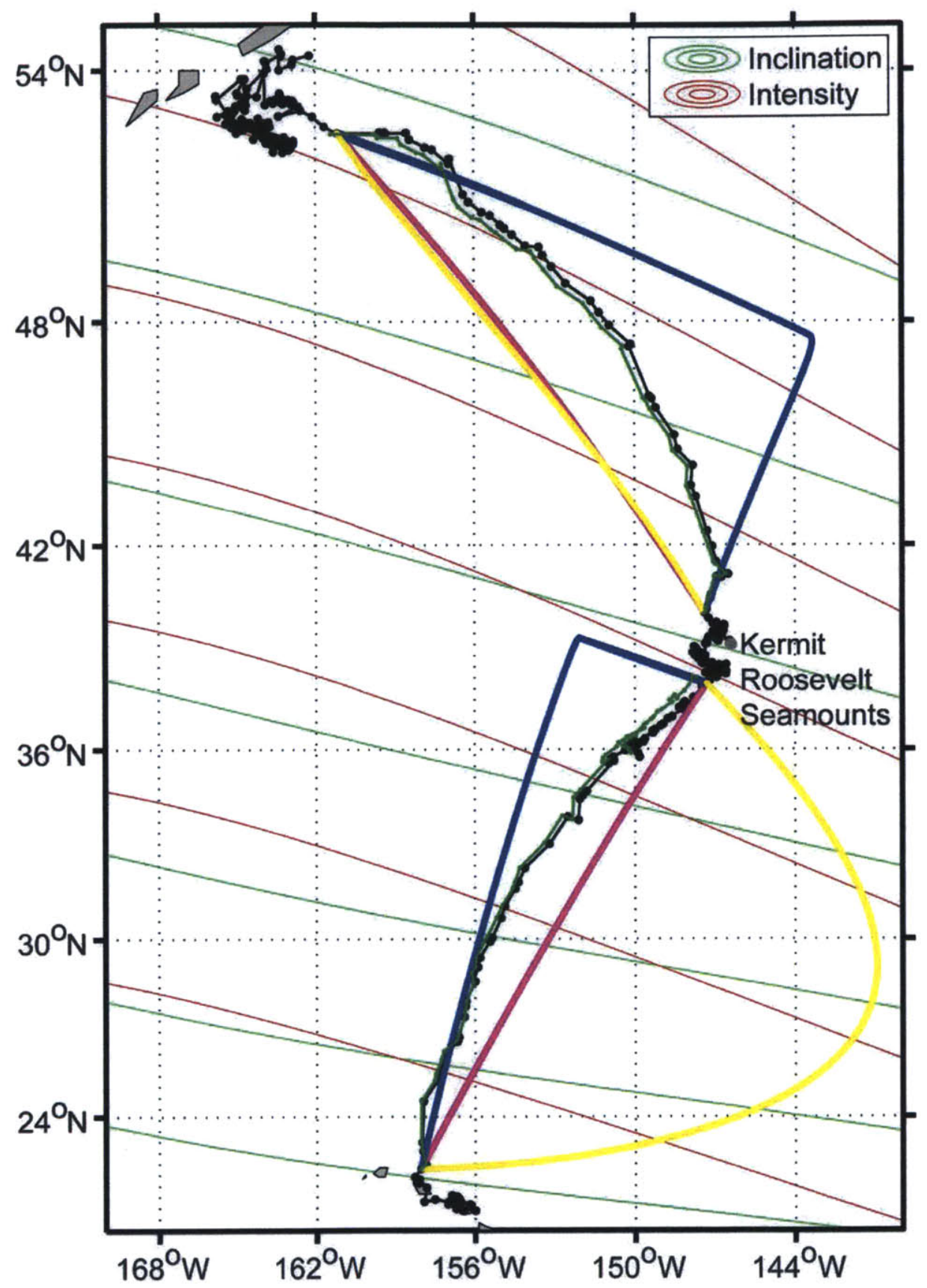

Figure 2.11: The track for Whale 8 plotted on a Mercator projection map. The color scheme is the same as in Figure 2.4. Whale 8 has the highest quality track of any of the whales. The whale first heads to the northeast, making an extended stop at the KermitRoosevelt Seamount, likely foraging on productivity created by upwelling in this region. Because of this stopover, and the significant change in direction afterwards, this track is split into two sections. In the first section the deviation of the observed track is to the 
west of the great circle route, and in the second section this deviation switches to the east of the great circle route. The bicoordinate model accurately predicts the deviation direction for both sections. Additionally, the bicoordinate route closely follows the observed track for the first half of the first leg, and for the start and end of the second leg. The complex bicoordinate route for the second half of this track is the only predicted track that closely matches the great circle route. When this whale reaches its destination near the Aleutian Islands, it switches quickly to movement patterns indicative of foraging.

The track for Whale 8 differs from those of the other tagged whales. This whale has a very steady course to the northeast, from the Hawaiian Islands to the KermitRoosevelt Seamount, where it then spends 37 days in a search pattern indicative of foraging; it then changes to a steady course to the northwest, reaching a destination quite close to that of whale 4, in southwest Alaska (Figure 2.11). Because of the significant change in direction, and the obvious extended stopover at the seamount, this track was broken up into two segments. The first segment starts as the whale leaves the Hawaiian Islands, and ends when it begins a search pattern near Kermit-Roosevelt Seamount. The second segment begins when the whale appears to initiate migration from the seamount area, and then home is chosen as the point where it transitions back to a search pattern near the Aleutian Islands (Figure 2.11). 


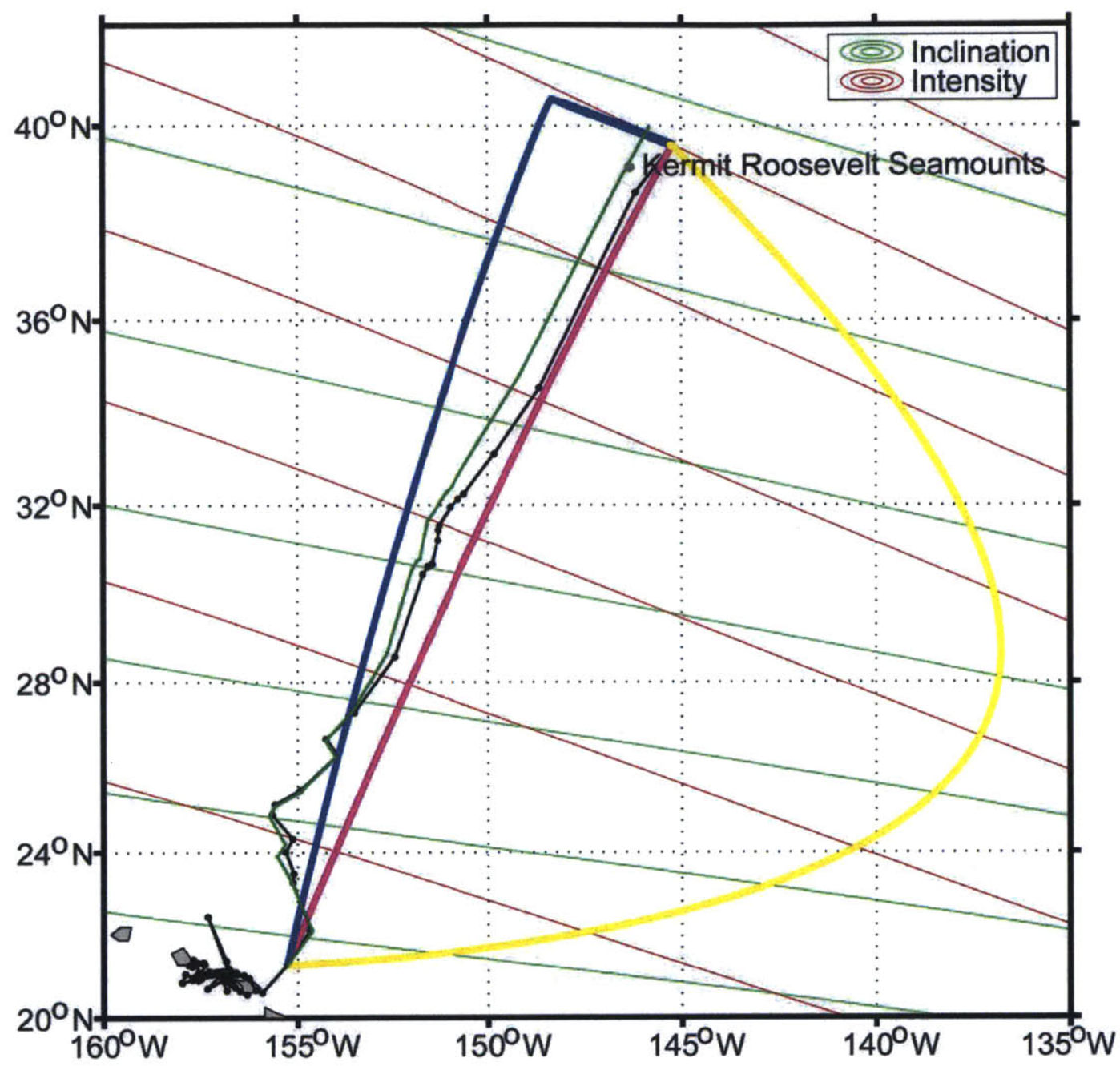

Figure 2.12: The track for Whale 9 plotted on a Mercator projection map. The color scheme is the same as in Figure 2.4. The deviation direction for this track matches that predicted by the bicoordinate model. The track does not deviate greatly from the great circle and has relatively few transit locations. This is one of three tracks that goes directly to the Kermit-Roosevelt Seamount before loss of tag transmissions. 


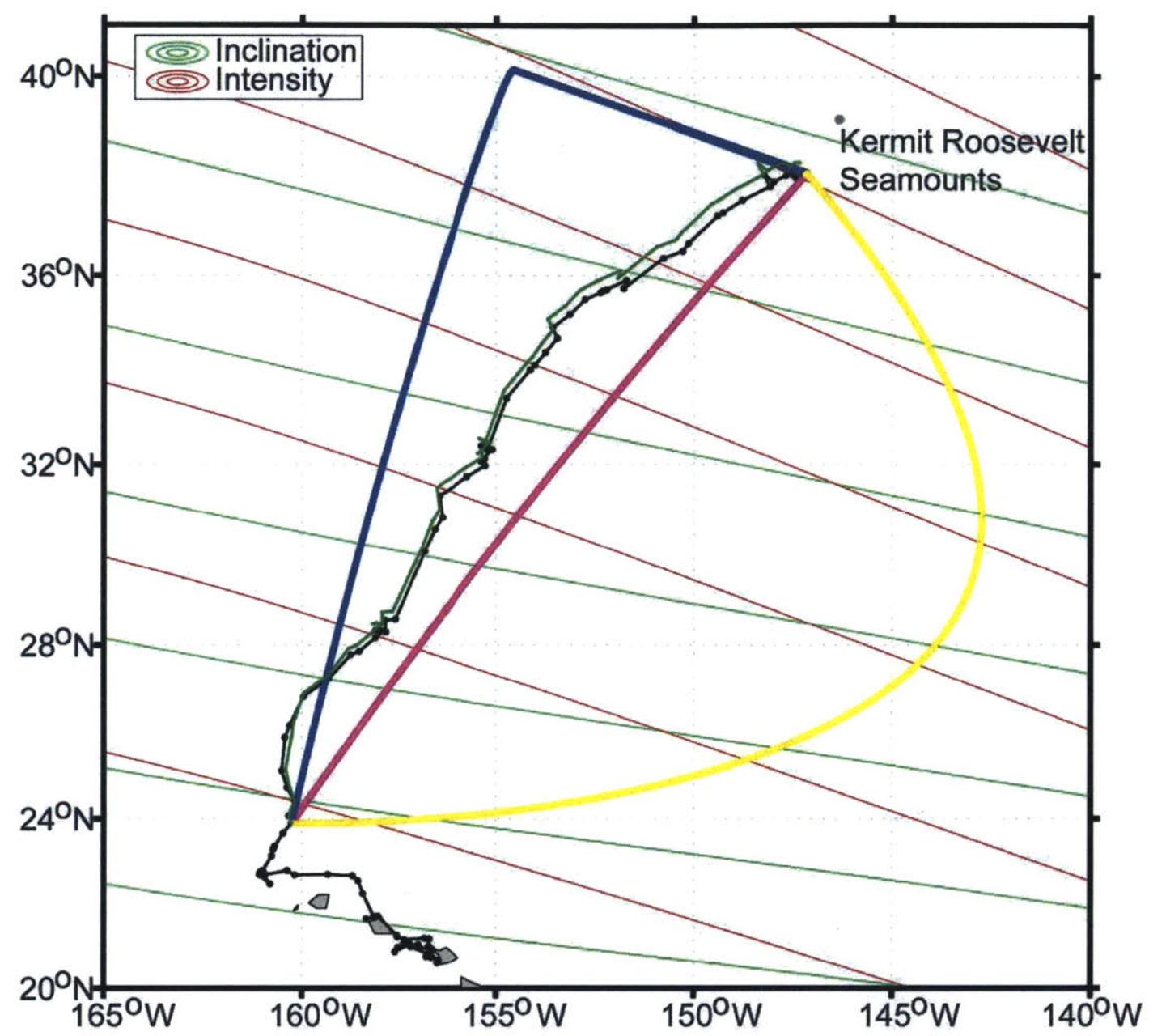

Figure 2.13: The track for Whale 10 plotted on a Mercator projection map. The color scheme is the same as in Figure 2.4. Whale 10 maintains a consistent deviation to the west of the great circle route. This deviation direction matches that predicted by the bicoordinate model. This whale appears to be on a direct route for the Kermit-Roosevelt Seamount, but the tag stops transmitting before it arrives.

When all of the tracks were plotted with a great circle route between their start and end locations (magenta line in track figures), it was found that most of them had a systematic deviation to one side or the other of this path. In the case of Whale 8, this deviation is to the west of the great circle route for the first half of the track. Once the animal departs the Kermit-Roosevelt Seamount and heads northwest, the track then switches to a deviation to the east of the great circle route (Figure 2.11). Whale 1 is the 
only track that closely resembles the great-circle route (Figure 2.4), and this is likely due to the fact that the track is relatively short. Several of the tracks initially follow a relatively straight course, before beginning the deviation from the straight-line course. This may be indicative of switching between navigation cues. Additionally, the home location is estimated as the last transmitted point for each whale that does not reach its destination. In this case, the short distance between the start and home location means that the whale would have to deviate sharply and significantly from the great circle route for the deviation direction to be detected.

In most of the tracks, the whales maintain a deviation to one side or the other of the great-circle route. However, Whale 7 first curves to the west of the great-circle route, before making an abrupt switch to the east of the great-circle about $1 / 4$ of the way to the destination (Figure 2.10). It then maintains a relatively steady heading for the middle portion of the track, although this may be biased by the small number of locations in this area. The track then curves back to the north in order to reach its destination.

\subsubsection{Comparison of bicoordinate geomagnetic tracks to observed tracks}

When the Benhamou (2003) bicoordinate model was used to plot a route between the start and end locations of each whale track (dark blue line in track figures), the direction of the deviation from the great circle route was found to correspond to the deviation direction in most of the whale tracks (Table 2.2). Most noticeably, the modeled deviation direction switches in the same manner as the observed track for Whale 8 (Figure 2.11). In the tracks of Whales 5,8 and 10, the modeled bicoordinate track closely corresponds to the observed track at either the start or the finish of the route. The observed track deviates away from the great-circle route in the opposite direction from the modeled bicoordinate track for only two whales, Whale 3 and Whale 6 (Table 2.2).

While the bicoordinate deviation direction matches the observed deviation direction in most cases, the magnitude of the deviation does not. In all cases, the modeled bicoordinate deviation is much greater than that of the observed tracks. In addition, all modeled tracks follow a relatively steady course until they reach the 
approximate home value for one of the fields, and then they make a sharp turn towards home. This occurs because the modeled headings are constrained to be between the two gradient field angles. Therefore, if the home direction is not between these two gradient angles, the track approaches the home field values until it reaches one of them, and then makes the sharp turn towards home by alternately moving up and then down the gradient fields, bouncing back and forth until the track reaches home. This feature is predicted by the model, with sharper turns present when the difference between the gradient field directions is small. In this region of the North Pacific, the difference between the two fields is on average about $8^{\circ}$, and therefore the change in direction is very sharp, close to $90^{\circ}$ in most cases. This feature is caused by the model constraints, and is not expected to ever occur in natural animal navigation, due to the influence of other navigational cues.

Table 2.2: A table of the direction each whale track deviated from the great circle path. The deviation direction of the modeled simple and true bicoordinate routes were then compared to the observed track deviation direction, and categorized according to whether or not they followed the initial deviation direction of the observed track (yes/no).

\begin{tabular}{|r|l|l|l|}
\hline Whale & Great Circle & $\begin{array}{l}\text { Simple } \\
\text { Bicoordinate }\end{array}$ & $\begin{array}{l}\text { True } \\
\text { Bicoordinate }\end{array}$ \\
\hline 1 & Match & N/A & N/A \\
\hline 2 & East & Yes & Yes \\
\hline 3 & West & No & No \\
\hline 4 & East & Yes & Yes \\
\hline 5 & West & Yes & No \\
\hline 6 & East & No & No \\
\hline 7 & West & Yes & No \\
\hline 8 & West/East & Yes & No \\
\hline 9 & West & Yes & No \\
\hline 10 & West & Yes & No \\
\hline
\end{tabular}

When the course for 'true' navigation via geomagnetic inclination and intensity was plotted for each track, the modeled course deviated substantially from the great circle route (yellow line in track figures). This was contrary to the prediction that this route would follow the straight-line course to the destination. The only case where the modeled course for true bicoordinate navigation closely matched the great circle route was during the second half of Whale 8's track (Figure 2.11). In all other cases, the 
modeled route deviates considerably from the great circle route, often in the opposite direction of that observed in the tracks. This discrepancy between the predicted and observed outcome is likely a result of the non-uniformity of the two fields and the distortion due to navigating on a sphere.

The simple bicoordinate model deviation direction matched the observed track deviation direction in 7 out of 9 tracks. A binomial test was then used to determine if this proportion of matching tracks was greater than expected by chance, i.e. if the probability of a track deviation matching a model deviation was greater than 0.5 . The probability that this proportion of matching tracks was greater than expected by chance was $\mathrm{P}=0.0898$, which is not statistically significant to an alpha level of 0.05 . However, this is likely due to the small sample size, which gives this test a power of only 0.37 , which means it has only a $37 \%$ chance of correctly rejecting the null hypothesis. To achieve a power of 0.8 with this proportion of successes and a 0.05 confidence interval, a minimum sample size of 18 whale tracks would be required. Only two out of nine tracks for the complex bicoordinate method matched the deviation direction of the observed track, and therefore this deviation is unlikely to occur more than would be expected by chance.

\subsubsection{Bicoordinate tracks for simulated fields}

The differences between the predicted complex bicoordinate tracks and the observed tracks led to verification of the calculations using simulated field parameters. The simulated fields and the modeled bicoordinate routes for several different combinations of gradient directions can be seen in Figure 2.14, Figure 2.15, and Figure 2.16. These simulations confirmed that the calculations were programmed correctly, as the deviations follow the pattern predicted, with sharper turns corresponding to smaller angles between the gradient directions (Figure 2.14). This is due to the fact that the modeled headings are constrained to be between the gradient field directions, therefore if this angle is small, the modeled heading stays between these two angles until it reaches the home value for one field, and then turns towards home by moving alternately up and down the gradients until it reaches the destination. 
The magnitude of the deviation from the straight-line route appears to be influenced not only by the angle between the gradient fields, but also by the angles of the gradient fields with respect to the home direction (Figure 2.14, Figure 2.15).

Additionally, the simulated field parameters demonstrate that the direction of the deviation appears to be solely based on the angles of the gradient directions in relation to the home direction (Figure 2.15). This is an important factor to consider when utilizing this method in the context of the deviation directions of the humpback whale tracks. 

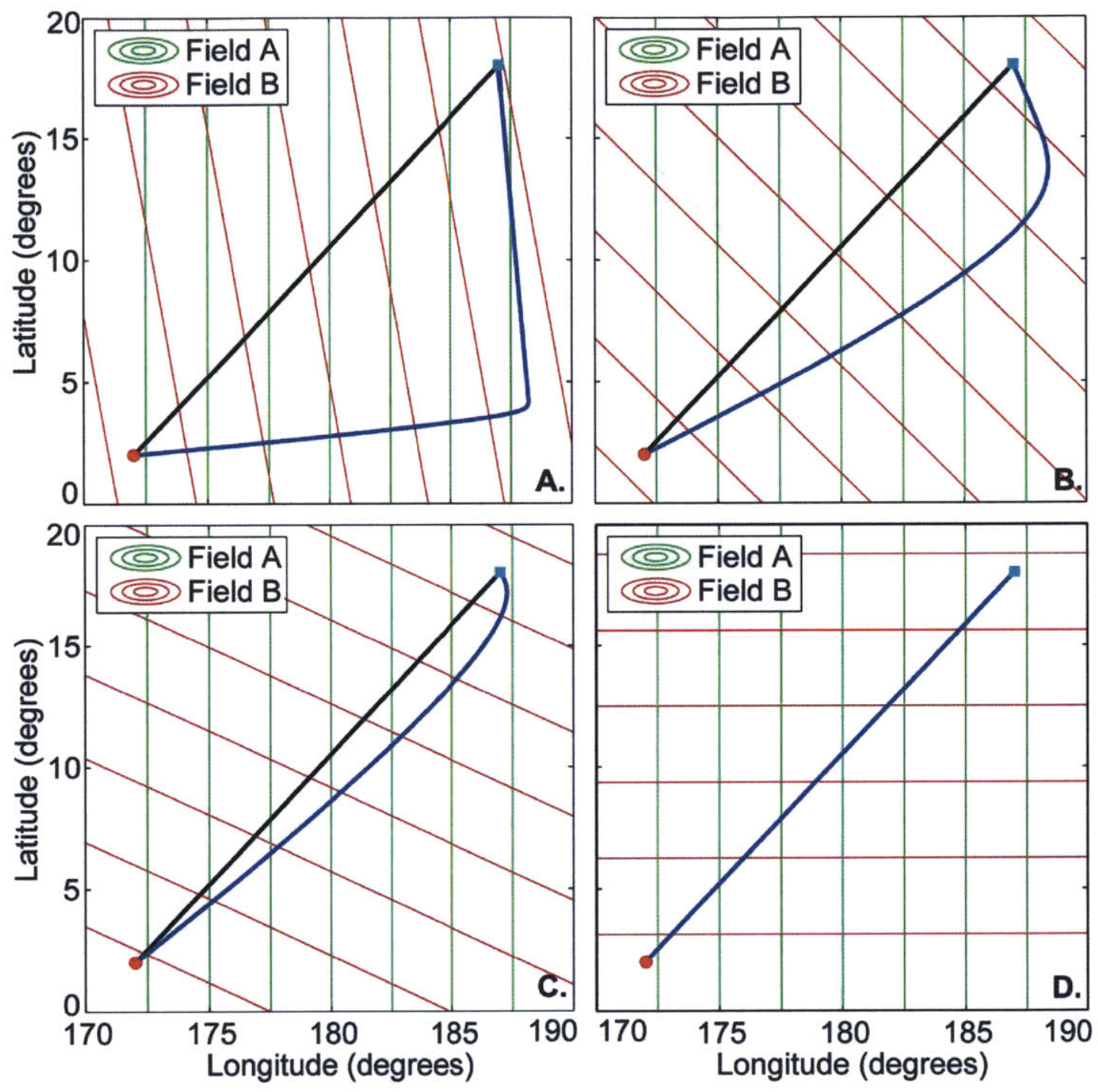

Figure 2.14: The straight-line (black) and modeled simple bicoordinate (blue) course for four different sets of simulated field angles; the start of the track is marked by the red circle and the end is marked by the light blue square. A.) Field $\mathrm{A}$ is set to $0^{\circ}$, Field $\mathrm{B}$ is set to $10^{\circ}$ counterclockwise from the east. B.) Field $\mathrm{A}$ is set to $0^{\circ}$ and Field $\mathrm{B}$ is set to $45^{\circ}$. C.) Field $A$ is set to $0^{\circ}$ and Field $B$ is set to $65^{\circ}$. D.) Field $A$ is set to $0^{\circ}$ and Field $B$ is set to $90^{\circ}$. The sharpness of the deviation angle is greater when the gradient field angles are close together. The bicoordinate course perfectly follows the straight-line course when the fields are orthogonal to each other (D.), as predicted by the Benhamou (2003) model. 

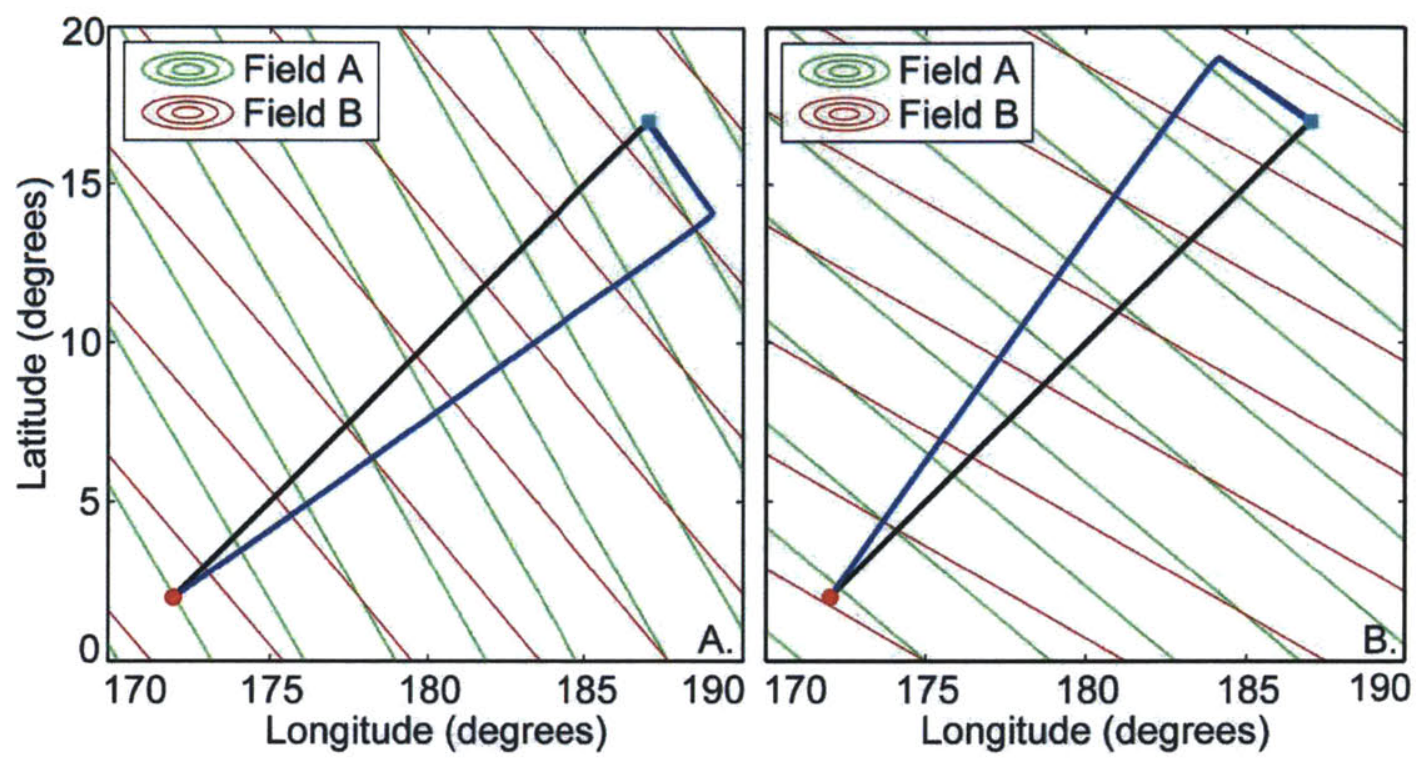

Figure 2.15: The modeled simple bicoordinate route based on simulated field parameters with the straight-line track marked in black and the bicoordinate in blue. The start of the track is marked by the red circle and the end is marked by the light blue square. In both A. and B. the angle between the two field gradients is the same $\left(10^{\circ}\right)$; this small angle between the two gradient field directions causes the sharp turn towards home. In A. both field angles are smaller than the track heading, $46^{\circ}$ counterclockwise from the east (Field $\mathrm{A}=30^{\circ}$, Field $\mathrm{B}=40^{\circ}$ from the east). In $\mathrm{B}$. both field angles are greater than the track heading (Field $\mathrm{A}=50^{\circ}$, Field $\mathrm{B}=60^{\circ}$ ). The direction of the deviation is determined solely by the field gradient angles in relation to the home direction.

As predicted, when the two fields were perfectly orthogonal to each other, the modeled route exactly follows the straight-line route (Figure 2.14). In addition, when the home direction is oriented exactly between the two field gradients, the modeled course also follows the straight-line route, even when the angle between the gradients is very small (Figure 2.16). When the method for 'true' bicoordinate navigation was modeled in the simulated fields, the bicoordinate course exactly followed the straight-line route, even when the angle between gradients was very small (Figure 2.17). This indicates that any deviations seen when using the true bicoordinate model with the real-world field parameters are due to either the non-uniformity of the fields, or the spherical nature of the earth's surface. 


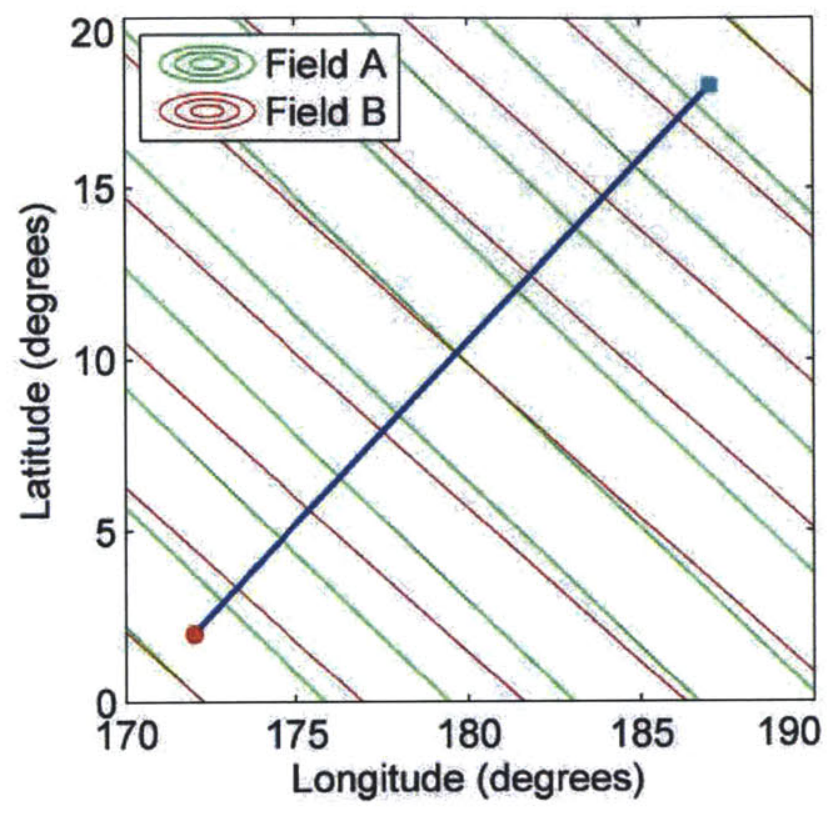

Figure 2.16: The modeled simple bicoordinate route, in which the home direction is perfectly centered between the two fields. The straight-line course is marked in black and bicoordinate course in blue. The start of the track is marked by the red circle and the end is marked by the light blue square. The track heading is $47^{\circ}$, the gradient direction of Field $\mathrm{A}$ is $46^{\circ}$, and Field $\mathrm{B}$ is $48^{\circ}$ counterclockwise from the east. Despite the fact that the gradient field directions are very close together $\left(2^{\circ}\right)$, the modeled route perfectly follows the straight-line route. 


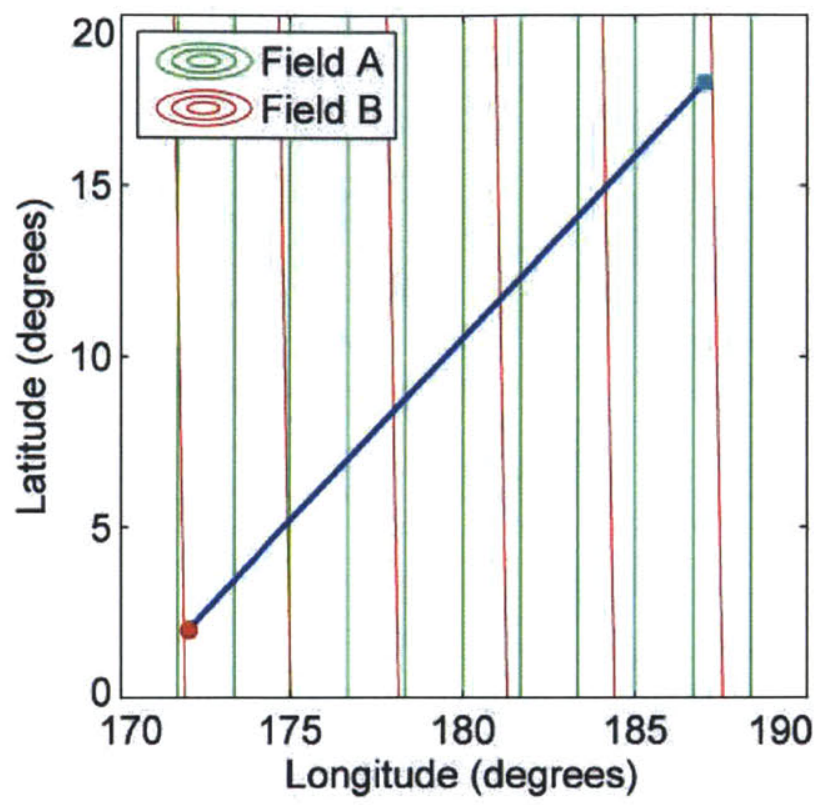

Figure 2.17: The modeled complex bicoordinate route, in which both fields are taken into account concurrently. The straight-line course is marked in black and the bicoordinate course in blue. The start of the track is marked by the red circle and the end is marked by the light blue square. As predicted, the modeled route perfectly follows the straight-line route, even in this case when the difference between the gradient field angles is very small $\left(1^{\circ}\right)$, and they are not oriented close to the home direction (Field $A=0^{\circ}$ and Field $\mathrm{B}=1^{\circ}$, counterclockwise from the east).

\subsubsection{Surface currents and heading adjustments}

When the influence of geostrophic surface currents was removed from the observed whale tracks (green route in track figures), there was no substantial change in any of the routes. The surface current speed was generally much smaller than the whales' swimming speeds, and therefore did not exert much influence on the heading of the whales. Initially, it was hypothesized that the deviations in the routes of the whales could be due to surface currents pushing them off an otherwise straight-line heading to their destination. However, when the current-corrected courses were plotted, in most cases the motor course deviated further from the great circle route than the observed course. This indicates that the currents were in fact pushing the whales in the direction of the great 
circle path. Therefore, any divergence from the straight-line path is a result of each whale's swimming heading, and is likely due to a navigational bias.

When the initial headings of the modeled and observed tracks were compared, there was no systematic bearing correction employed by the whales (Figure 2.18). For high quality tracks, such as Whale 8 , where the track follows a smooth deviation, the heading difference was smaller at the start of the track and greater towards the end, as expected. When the circular mean and standard deviation of these heading differences were calculated, the standard deviation for each track ranged from $\pm 21.8^{\circ}$ to $\pm 55.4^{\circ}$. If there was a steady heading correction, the standard deviation would be close to zero, as the whale would maintain a set bearing away from the model bearing.

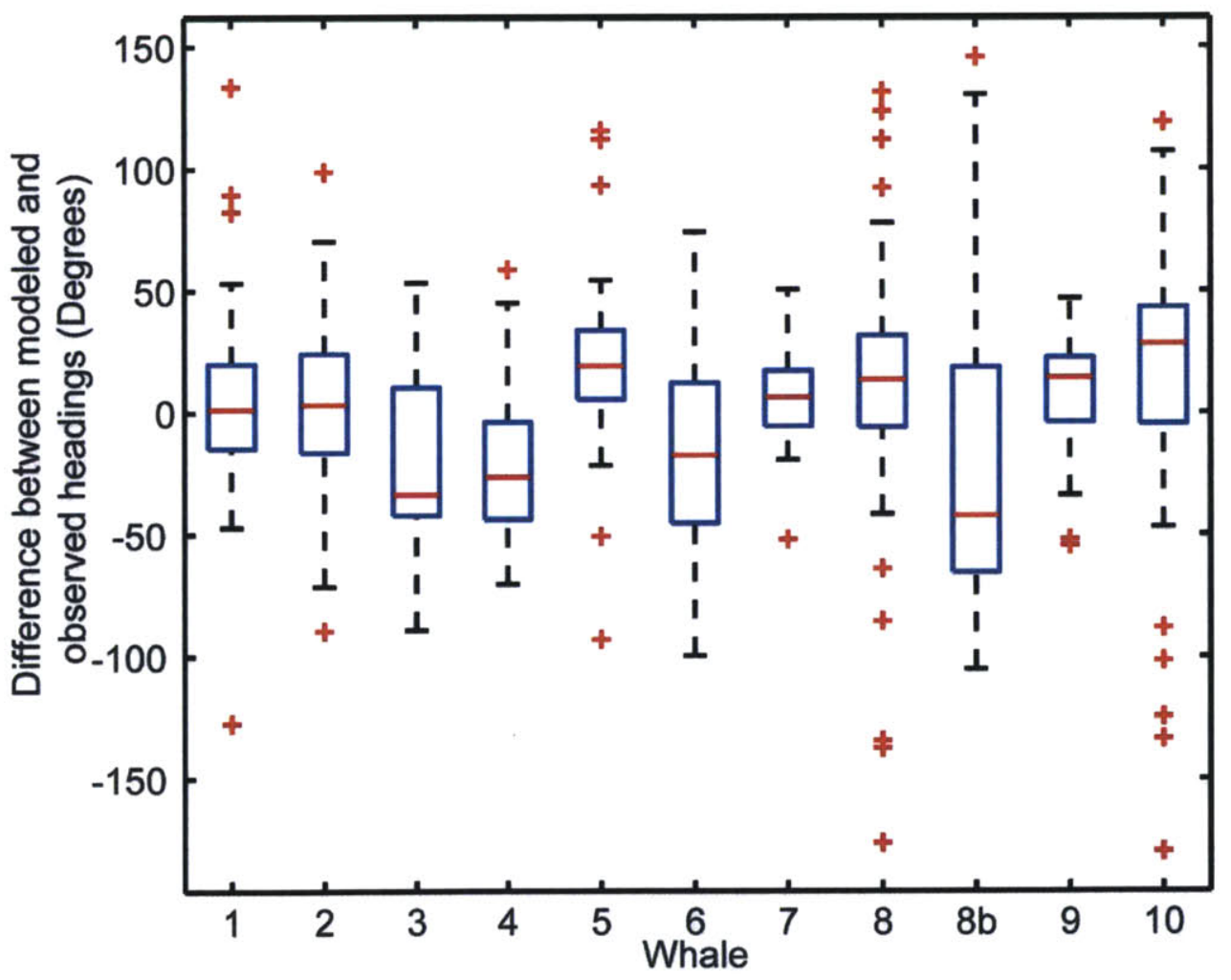

Figure 2.18: Box plot of the differences between the observed and modeled heading for each whale (the Whale 8 track is split into two parts). The central red line is the median, the edges of each box are the 25 th and 75 th percentiles. Whiskers from the box extend to the most extreme data points not considered outliers, and the outliers are plotted individually in red. In all cases the variation around the mean heading is quite large. 


\subsection{Discussion}

\subsubsection{General navigation performance}

This study utilizes the tracks of whales satellite-tagged in Hawaii to demonstrate that humpback whales are capable of accurate trans-oceanic migrations. This population of humpbacks, like many populations of baleen whales, crosses vast ocean distances twice a year in order to reach their feeding or breeding grounds. In this study, the whales were tagged in the Hawaiian Islands before their migration to feeding destinations in Alaska and British Columbia. This spring migration presents a potentially less difficult navigation task than the reverse migration, as the whales are migrating to a long coastline rather than an archipelago. An animal migrating to a feeding ground located on a coastline could use a single cue as a compass bearing to find the coastline and then swim either up or down the coast to its destination (Lohmann et al. 2007). While the humpback whales tagged in this study could utilize vector navigation to reach their feeding grounds along the North American coastline, the reverse migration of this population to Hawaii requires precise navigation mechanisms. While it is potentially possible to find an island utilizing vector navigation (Lohmann et al. 2007), this is an imprecise method of navigation and could very easily lead an animal to miss its destination when it is presented with any deflecting forces, such as ocean currents. Therefore, it is quite likely that this population of humpback whales, which migrates both to the coastline of North America and back to the islands of Hawaii, possesses some form of true navigation.

By migrating to the Kermit-Roosevelt Seamount area, Whales 8 and 9 demonstrate the ability to locate a small target. Very few navigation cues could lead an animal to this precise location in the ocean. Yet, several of the whales still manage to reach this location, with Whale 8 demonstrating a steady course to this destination, with no apparent search pattern employed to find it (Figure 2.11). However, it is possible that the whales are not in fact heading directly for the Kermit-Roosevelt Seamount, but instead set a course in that general direction in the hopes of finding it along their way. For a whale migrating from Hawaii to British Columbia, the seamounts would be roughly along the way, and there would be no major cost to missing it, as the whale would 
eventually reach the final feeding destination. While this could have been the case for Whale 9 (Figure 2.12), the final destination for Whale 8 was in the Aleutian Islands in southwest Alaska. There is relatively little interchange of individuals among feeding areas (Calambokidis et al. 2001); therefore this whale made a significant deviation away from a route to its final destination, in order to stop at the Kermit-Roosevelt Seamounts for well over a month. This suggests that the whale was willing and capable of making a significant deviation from its final destination to an out of the way, difficult to locate stopover. This implies that there was no great risk of failure, otherwise such a deviation would likely not be feasible.

Despite the fact that the whales are apparently capable of navigating to precise locations, in none of the satellite tracks of this study did the whales follow the most efficient, great circle route. Considering that the whales fast during their migration, and that it is an energetically costly trip, there is significant pressure on them to make this trip as efficiently as possible (Lockyer 1981, Kshatriya and Blake 1988). However, in every case, the actual route the whale took was longer than the most efficient, great circle route (Table 2.1). In almost all cases, this extra distance was due to a systematic deviation to either the east or the west of the great circle route. If it is assumed that the whales are attempting to follow the most direct route, then these deviations are likely the result of either deflecting currents, or a systematic navigation error from the cues they are utilizing.

When the influences of geostrophic surface currents were removed from all of the whale tracks (dark green tracks Figure 2.4 to Figure 2.13), the adjusted tracks did not differ greatly from the observed tracks. In most cases, the current-corrected tracks actually deviated more from the great circle path than the observed track. This indicates that the currents were in fact pushing the whales towards the great circle route, and the actual motor headings of the whales deviated from the great circle slightly more than is indicated by the observed track. Therefore, the effects of deflecting currents can be ruled out, and the most likely explanation for the deviations from the great circle route is a systematic navigation bias introduced by the navigation cues. This means that comparing 
the path each whale took with the path predicted by following a specific cue could give insights into which cues the whales may be using.

\subsubsection{Correlation between modeled and observed deviation directions}

Benhamou (2003) presents a method to model the path an animal would take when following two gradient fields to a destination. Benhamou (2003) does not discuss the specifics of which navigation cues would be appropriate for this method, but instead presents a general method that can be applied to any two gradient fields. However, as discussed above, the prevailing opinion in the animal navigation literature is that the inclination and intensity of the earth's magnetic field are the only universally present cues that can provide both a map and compass for use in true navigation (Lohmann and Lohmann 1996b, Lohmann et al. 2007). Both of these parameters form gradient fields, with inclination varying roughly with latitude, and intensity varying less uniformly. Therefore, these two field features were the logical choice for modeling a course for each whale based on geomagnetic cues.

When the path for each whale was modeled according to the methods of Benhamou (2003), utilizing the inclination and intensity of the magnetic field, the direction of the deviation from the great circle route matched the deviation in the observed tracks in 7 out of 9 cases. When a binomial test was used to compare the proportion of matching tracks to the proportion expected by chance $(50 \%)$, the probability that this proportion of matching tracks was greater than expected by chance was $\mathrm{P}=0.0898$, which is not statistically significant to an alpha level of 0.05 . However, the small sample size in this study means that the power of this test is very small (power $=0.37$ ). This means that there is only a $37 \%$ probability of being able to determine that the number of matching tracks is greater than expected by chance. In order to get a test with a power of 0.8 , with this percentage of matching tracks, a minimum sample size of 18 whales would be required. However, the observed trend provides some support for the hypothesis that the whales are utilizing magnetic inclination and intensity to navigate to their destinations, and there is still a significant amount of information that can be 
obtained by comparison of the observed tracks from this study with the tracks modeled according to bicoordinate geomagnetic navigation.

\subsubsection{Routes modeled with simulated field parameters}

Modeling the deviations that would be observed under simulated field parameters gave significant insight into the methods of Benhamou (2003), as well as to the factors that determine the pattern observed in each of the modeled courses. The simulated tracks in Figure 2.14 demonstrate that, as predicted by Benhamou (2003), the smoothness of the deviation from the straight course is entirely dependent upon the angle between the two gradient fields, with a nearly $90^{\circ}$ turn in the track when the fields are only $10^{\circ}$ apart (Figure 2.14 part A). This angle lessens as the two fields are rotated further apart, with a completely straight line between the start and end points achieved when the two fields are orthogonal to each other (Figure 2.14 part B). This follows the pattern predicted, and indicates that the real-world model, which was programmed in the same way as the simulated model, was calculated correctly.

What is not apparent in these figures is that the location of this inflection point is dependent on the gradient directions of Field A and B in relation to the home, with gradient directions closer to the home bearing having an inflection point closer to home. Perhaps most telling is that the direction of the deviation depends entirely on the direction of the field gradients in relation to the home bearing (Figure 2.15). This means that the modeled deviation direction seen for each track is the result of the exact combination of the gradient directions of the geomagnetic inclination and intensity in relation to each home direction. This is seen most obviously in the switching of the modeled deviation direction of Whale 8 (Figure 2.11), in which the field directions remain roughly the same, but the home direction of the whale changes dramatically. Additionally, the fact that the given patterns only occur under the very specific field conditions seen for each track increases the likelihood that the similarities between the observed and modeled tracks are not simple happenstance. 
Further, this means that the choice of home can have a large influence on the deviation direction. Each home location was chosen before the model was implemented, and therefore the home choice was not influenced by this factor. The two tracks for which the observed deviation does not match the modeled deviation (Whale 3 and 6) are both among the shorter, lower resolution tracks, as well as the only two that have significant initial movement to the northwest. The choice of home as the last transmitted point could have had a large influence on the deviation direction seen in these tracks. The possibility that the deviations would match if the tag lasted until the final destination cannot be ruled out.

However, this also means that choice of home can have a large influence on those tracks that do not reach their destinations but for which the modeled and observed track do match. The likelihood of the home destination changing dramatically in relation to the fields is reduced for the longer tracks, as they are unlikely to switch sharply to a new destination once they are most of the way across the ocean. The exception to this is a case such as Whale 8, where there is a stopover destination partway through the migration. However, in a case like this, the migration would be split between the two destinations, as was done with Whale 8 , in which case the route to the stopover destination would still be valid.

Benhamou (2003) suggests the simplified version of bicoordinate navigation as an alternative to the 'correct' true navigation achieved by taking both fields into account at once. When this complex method was modeled using real-world values for geomagnetic inclination and intensity, it was found that, contrary to the prediction, the modeled tracks showed large deviations from the great circle routes in all cases except for the second half of the track for Whale 8. These deviations were quite large, and in most cases, actually in the opposite direction of the observed deviation. These large discrepancies between the predicted and actual results led to a necessary verification of the model calculations. This was done by using the real-world model calculations with simulated uniform field parameters on a flat plane. When these tracks were modeled, the calculations themselves were found to be correct, and the modeled route matched the straight-line route under all 
field angles (Figure 2.17). This means that these large deviations are due to either the non-uniformity of the field gradients in this region, or the spherical nature of the earth. Regardless of which factor influences these discrepancies, these simulations confirm that the complex 'true' navigation method of Benhamou (2003) is not in fact applicable to all real-world animal migrations, and may have problems with field patterns in some areas of the world.

While the complex model did not follow predictions, the simple model behaved as expected given the geomagnetic field parameters and destinations. The deviation direction matched the observed deviations in 7 out of 9 cases, but the magnitude of the modeled deviation was greater than that of the observed tracks in all cases. It was found that this difference was not due to the whales maintaining a set correction away from the geomagnetic bicoordinate route. Therefore, I believe that this improved accuracy is likely due to the whales using other navigation cues, in addition to geomagnetic inclination and intensity, to inform their routes.

\subsubsection{Use of multiple cues during migration}

All migrating animals studied thus far have been found to rely on multiple sources of information when navigating (Lohmann and Lohmann 1996a, Avens and Lohmann 2003, Cochran et al. 2004, Muheim et al. 2006, Lohmann et al. 2008b). This makes sense from an evolutionary perspective, both in the context of one cue providing insufficient resolution for an entire migration, as well as the high vulnerability of an animal if this single sense is disrupted. While the earth's magnetic field provides worldwide, omnipresent information that can be utilized for navigation, it is not without sources of error, which can at times be quite large. The main drawback of the magnetic bicoordinate navigation theory is that there are regions of the earth where isolines of inclination and intensity are extremely close together (angular difference of $\leq 2^{\circ}$ ) (Figure 2.19) (Boström et al. 2012). When the field lines are this close together, there is a mirror effect, where a particular magnetic bicoordinate combination occurs on both sides of the area (Boström et al. 2012). If an animal were using only inclination and intensity to 
navigate, this could cause it to migrate to the wrong combination of grid intersections. In the area of this study, the isolines of inclination and intensity are on average about $8^{\circ}$ apart. While this does not lead to mirroring of the coordinates, it can result in the large deviations seen in the modeled bicoordinate tracks. It is often suggested that in these zones of close magnetic isolines, an animal could detect latitude, but not longitude. However, loggerhead turtles have been shown to use inclination and intensity to determine their longitude, even when these field lines are very close together (Putman et al. 2011).

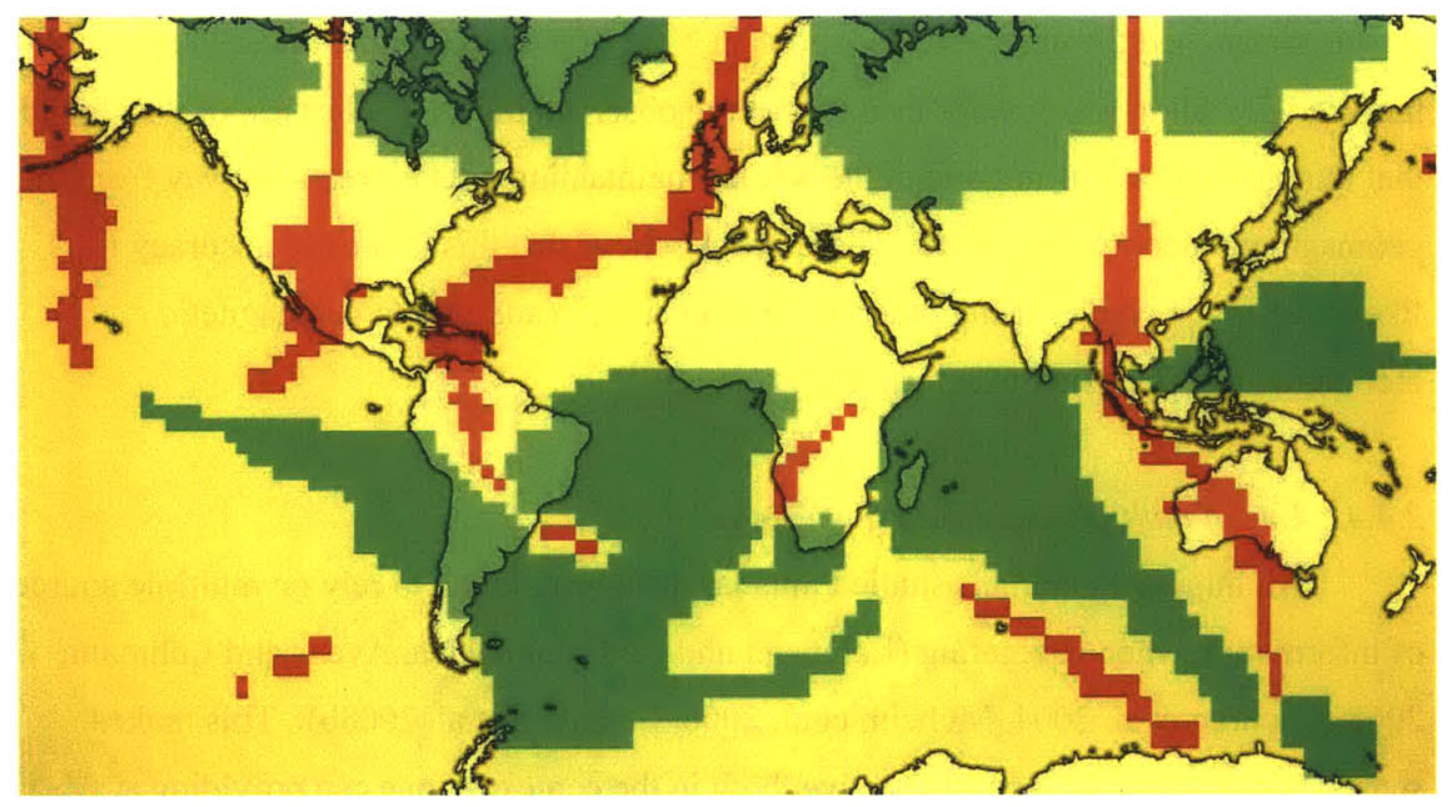

Figure 2.18: Figure from Boström et al. (2012) illustrating the angular difference between isolines of total field intensity and inclination. Areas with an angular difference of $\leq 2^{\circ}$ are shown in red, $2-30^{\circ}$ in yellow and $\geq 30^{\circ}$ in green.

Both the problem of duplication of magnetic bicoordinates, and the large deviations due to small angles between field gradients, can be addressed by the addition of other navigational cues. In both of these cases, a simple compass orientation could resolve any ambiguity in the destination, as well as allow for more efficient homing. Compass information is available to a migrating animal from multiple sources including the sun, magnetic polarity, celestial cues, and potentially even sound. 
The sharp turn seen in the modeled bicoordinate track is the result of the method constraining the track to be between the two field gradient directions. Therefore, when the modeled animal reaches the destination value for one field, it then alternates going up and down the fields until it reaches the destination. An animal actually applying this technique would instead follow the two fields until it reached the correct isoline of one, and then follow that isoline to its intersection with the other home field coordinate. However, in nature, an animal is not constrained to headings between the gradient field directions. The addition of a compass direction to the magnetic inclination and intensity information could allow an animal to determine when bicoordinate navigation is leading it far astray, and to partially correct for this deviation. In this way, the magnetic inclination and intensity provide the animal with information on its position in relation to home, allowing for homing even in the face of deflections, while an added compass direction would allow it to correct some for the deviations introduced by this method.

While other cues, in addition to the two geomagnetic fields, could help an animal to correct for the deviations of bicoordinate magnetic navigation, they are also necessary to provide backup navigational information in the case of failure of the primary mechanism. Both birds and turtles have been shown to utilize celestial cues to recalibrate their magnetic sense when it is experimentally disrupted (Avens and Lohmann 2003, Cochran et al. 2004). The earth's magnetic field is subject to both short term and long term disruptions, both of which could be devastating to a migratory species that only utilizes geomagnetic navigation information. Across the globe, there are areas of geomagnetic anomalies, where the magnetic intensity deviates markedly from the regional field due to variation in the magnetic properties of underground rock formations. These anomalies could disrupt the navigation of an animal using intensity as one bicoordinate magnetic cue to cross these areas. Additionally, the earth's magnetic field is believed to completely reverse polarity every 0.1 to 1 million years. While this phenomenon occurs slowly on the time scale of biological life, it is still a factor that could affect a species as a whole. However, there are studies showing that birds and turtles are capable of utilizing sun cues to recalibrate their magnetic receptors on a daily 
basis (Avens and Lohmann 2003, Cochran et al. 2004). By using one cue to recalibrate another, an animal can ensure that it will be able to successfully navigate even when there is a disruption to one source of information.

While a whale could be using multiple sources of information in this way, to both calibrate and correct for geomagnetic navigation, it is also likely that they are utilizing different navigation cues on different spatial scales along their migration. Almost all studies of animal navigation agree that animals are likely using all sources of orientation information available to them, both simultaneously checking cues against each other as well as switching between different cues at different spatial scales (Wiltschko and Wiltschko 2003, Bingman and Cheng 2005). As stated above, there are many different cues that provide different resolutions of orientation information, including: geomagnetic cues, sun and other celestial cues, air or water borne chemical cues, and physical features of the ocean itself. While magnetic cues may allow an animal to navigate over large distances, as the animal approaches its destination, new orientation information will be available, such as chemical cues or wave orientation. These cues may provide information specific to the destination, but they also have a limited range. Therefore, if an animal can use a less specific, but farther-ranging signal to approach its destination, it could then switch to more specific cues to home in, once these come within range. An example of this type of cue switching is seen in hatchling sea turtles that utilize the bright seaward horizon to find their way to the water, then initiate seaward movement by orienting into the waves, then switch to magnetic and sun cues as they enter the open ocean (Lohmann and Lohmann 1996a). This process can be employed in reverse, with successively finer-scale cues leading them to a particular island. This type of cue switching may explain some of the features seen in the humpback whale tracks.

Several of the whale tracks in this study have initially straight courses as they leave the Hawaiian Islands. This could be due to the whales utilizing a single compass cue to guide orientation away from the island. Additionally, seven of the whales pass through the Musicians Seamounts Trail to the north of Hawaii. It is possible that regions such as this serve as waypoints that the animals first travel to before switching to other 
cues, or perhaps as a point where they line up specific cues they utilize for the transoceanic portion of their migration. Additionally, many of the tracks have inflection points where the whales are seemingly following one heading and then switch to another heading. It is possible that this is indicative of the animals switching between cues and thus introducing a slightly different bias to the track. There are many interesting features of this nature in these whale tracks, which could provide insight into the different spatial scales of whale movement, but none other than geomagnetic bicoordinate navigation are empirically tested here.

The likelihood that the whales are using all available orientation information does not negate the possible importance of geomagnetic information. However, it could easily explain the discrepancies between the observed and modeled tracks. If the whales are following a magnetic bicoordinate route, but using outside information to improve their courses, this could explain why the deviation direction, but not the deviation magnitude, matches in many of the whale tracks.

\subsection{Conclusions}

This study compares the migration tracks of North Pacific humpback whales to migration tracks modeled according to navigation via geomagnetic inclination and intensity. When the direction of the deviation from the great circle route for both the observed and modeled tracks were compared, they were found to match in 7 out of 9 cases. The small sample size means that any statistical tests have low power. However, the high percentage of matching tracks suggests that there could be a correlation between the observed and modeled tracks, which would indicate that geomagnetic cues are important for humpback whale navigation. Using this same method with double the sample size would allow for a more accurate assessment of the significance of the percentage of matching tracks.

While the direction of the deviations matched in many of the tracks, the magnitude of the deviation did not. This suggests that even if the whales use geomagnetic cues to navigation, that they are also utilizing other navigation cues to 
improve their orientation. This could allow for improved accuracy, as well as redundancies in the navigation system during times of cue inaccuracy or unavailability.

Additionally, this study illustrates that the theoretical simple method proposed by Benhamou (2003), by which an animal can navigate by taking two gradient fields into account individually could be used for navigation by an animal in the North Pacific using geomagnetic inclination and intensity. The complex method of navigation, suggested by Benhamou (2003) to be too complicated for animal use, was furthermore shown to be problematic when applied to the gradient fields of geomagnetic inclination and intensity in this study location. By contrast, the simple method of bicoordinate navigation he proposed is both applicable to long distance navigation, and potentially simple to employ. This study demonstrates that modeling the migration tracks based on a pair of navigation cues can provide useful information about the underlying navigation mechanisms of species that are not easily manipulated in experiments. These methods have provided some initial insights into the plausibility of bicoordinate geomagnetic navigation in humpback whales.

However, this chapter only looks at two possible methods of bicoordinate geomagnetic navigation. Further investigation should focus on other potential methods of bicoordinate navigation that could lead to a more efficient track. An animal that makes multiple migrations across a given area could learn over the course of several migrations how the geomagnetic cues lead it away from the most efficient route, and then potentially learn to correct for the deviation. In addition, an animal that knows how the gradient fields change across its course could optimize the rate that it traverses each field gradient, rather than simply calculating the route directly up or down each gradient, such that it will reach the home values for each field at the same time. This type of rate optimization could be explored in the future with modeling studies. Additional future work should focus on integrating a compass direction into the bicoordinate model, and determining if this leads to a migration track that more closely resembles the observed tracks. This could be expanded out to a general inverse model in which each navigation 
cue is added to a model, until the modeled track approaches an approximation of the observed track.

When several models are established in this manner, a quantitative measure is needed in order to assess which track most closely resembles the observed data, and therefore may most closely approximate the actual methods used by the whales. Two ways to look at the fit of the model to the data would be to either calculate the distances between the modeled and observed track at corresponding time points or by summing the total area between the modeled and observed track. These distances would give an estimate of how closely the modeled track follows the observed track, and therefore which model most closely approximates the whale's actual navigation system.

Future work should also focus on establishing whether or not whales possess a geomagnetic sense. While experiments studying the behavior of the animals when the geomagnetic field is disrupted would be helpful, they are logistically difficult to conduct. However, a correlation study, similar to that of Kirschevink et al. (1986) and Klinowska (1986), in which worldwide marine mammal stranding data is correlated to geomagnetic anomalies, could provide further evidence of a magnetic sense in whales.

Therefore, while whales are difficult experimental subjects, there are many potential avenues of investigation that could shed light on the navigation methods they employ. With constantly improving satellite data that allows scientists to follow whales on their migrations, the methods of cetacean navigation should now be an area open to detailed future investigation.

\subsection{Acknowledgements}

This work would not have been possible without Dr. Bruce Mate's collaboration and generous sharing of the humpback whale tracks. Dr. Benjamin Hodges and Dr. Mark Baumgartner provided essential assistance in implementing and refining the MATLAB program for the model. My entire thesis committee provided helpful comments and insights for this project, with particular guidance along the way from my adviser, Dr. Peter Tyack. 


\subsection{References}

Able, K. P. 2001. The concepts and terminology of bird navigation. Journal of Avian Biology 32:174-183.

Amante, C. and B. W. Eakins. 2009. Etopol 1 arc-minute global relief model: Procedures, data sources and analysis. NOAA Technical Memorandum NESDIS NGDC24.

Argos. 2011. Guide to the argos system. Pages 68 CLS, Toulouse.

Avens, L. and K. J. Lohmann. 2003. Use of multiple orientation cues by juvenile loggerhead sea turtles Caretta caretta. Journal of Experimental Biology 206:4317-4325.

Belkin, I. M., P. C. Cornillon and K. Sherman. 2009. Fronts in large marine ecosystems. Progress In Oceanography 81:223-236.

Benhamou, S. 2003. Bicoordinate navigation based on non-orthogonal gradient fields. Journal of Theoretical Biology 225:235-239.

Benhamou, S., J. Sudre, J. Bourjea, S. Ciccione, A. De Santis and P. Luschi. 2011. The role of geomagnetic cues in green turtle open sea navigation. PLoS ONE 6:e26672.

Berens, P. 2009. Circstat: A matlab toolbox for circular statistics. Journal of Statistical Software 31:25-27.

Bingman, V. P. and K. Cheng. 2005. Mechanisms of animal global navigation: Comparative perspectives and enduring challenges. Ethology, Ecology \& Evolution $17: 295$.

Boles, L. C. and K. J. Lohmann. 2003. True navigation and magnetic maps in spiny lobsters. Nature 421:60-63.

Boström, J. E., S. Åkesson and T. Alerstam. 2012. Where on earth can animals use a geomagnetic bi-coordinate map for navigation? Ecography 35:1039-1047.

Calambokidis, J., G. H. Steiger, J. M. Straley, L. M. Herman, S. Cerchio, D. R. Salden, U. R. Jorge, J. K. Jacobsen, O. V. Ziegesar and K. C. Balcomb. 2001. Movements and population structure of humpback whales in the North Pacific. Marine Mammal Science 17:769-794.

Cochran, W. W., H. Mouritsen and M. Wikelski. 2004. Migrating songbirds recalibrate their magnetic compass daily from twilight cues. Science 304:405-408. 
Deutschlander, M. E., M. J. Freake, S. C. Borland, J. B. Phillips, R. C. Madden, L. E. Anderson and B. W. Wilson. 2003. Learned magnetic compass orientation by the siberian hamster, Phodopus sungorus. Animal Behaviour 65:779-786.

Dolphin, W. F. 1987. Dive behavior and estimated energy expenditure of foraging humpback whales in Southeast Alaska. Canadian Journal of Zoology 65:354-362.

Fancy, S. G., L. F. Pank, D. C. Douglas, C. H. Curby and G. W. Garner. 1988. Satellite telemetry: A new tool for wildlife research and management. DTIC Document. $61 \mathrm{pp}$.

Finlay, C., S. Maus, C. Beggan, T. Bondar, A. Chambodut, T. Chernova, A. Chulliat, V. Golovkov, B. Hamilton and M. Hamoudi. 2010. International geomagnetic reference field: The eleventh generation. Geophysical Journal International 183:1216-1230.

Fischer, J., M. Freake, S. Borland and J. Phillips. 2001. Evidence for the use of magnetic map information by an amphibian. Animal Behaviour 62:1-10.

Gabriele, C. M., J. M. Straley, L. M. Herman and R. J. Coleman. 1996. Fastest documented migration of a North Pacific humpback whale. Marine Mammal Science $12: 457-464$.

Gendron, D. and J. Urban. 1993. Evidence of feeding by humpback whales (Megaptera novaeangliae) in the Baja California breeding ground, Mexico. Marine Mammal Science 9:76-81.

Holland, R. A., K. Thorup, M. J. Vonhof, W. W. Cochran and M. Wikelski. 2006. Navigation: Bat orientation using earth's magnetic field. Nature 444:702-702.

Horton, T. W., R. N. Holdaway, A. N. Zerbini, N. Hauser, C. Garrigue, A. Andriolo and P. J. Clapham. 2011. Straight as an arrow: Humpback whales swim constant course tracks during long-distance migration. Biology Letters 7:674-679.

Kirschvink, J. L., A. E. Dizon and J. A. Westphal. 1986. Evidence from strandings for geomagnetic sensitivity in cetaceans. Journal of Experimental Biology 120:1-24.

Klinowska, M. 1986. Cetacean live stranding dates relate to geomagnetic disturbances. Aquatic Mammals 11:109.

Kobayashi, D. R., J. J. Polovina, D. M. Parker, N. Kamezaki, I. J. Cheng, I. Uchida, P. H. Dutton and G. H. Balazs. 2008. Pelagic habitat characterization of loggerhead sea turtles, Caretta caretta, in the North Pacific ocean (1997-2006): Insights from satellite tag tracking and remotely sensed data. Journal of Experimental Marine Biology and Ecology 356:96-114. 
Kshatriya, M. and R. Blake. 1988. Theoretical model of migration energetics in the blue whale, Balaenoptera musculus. Journal of Theoretical Biology 133:479-498.

Light, P., M. Salmon and K. J. Lohmann. 1993. Geomagnetic orientation of loggerhead sea turtles: Evidence for an inclination compass. Journal of Experimental Biology 182:110.

Lockyer, C. 1981. Growth and energy budgets of large baleen whales from the southern hemisphere. Pages 379-487 in Mammals in the seas. Food and Agricultural Organization of the United Nations, Rome.

Lohmann, K. and C. Lohmann. 1994. Detection of magnetic inclination angle by sea turtles: A possible mechanism for determining latitude. Journal of Experimental Biology 194:23-32.

Lohmann, K. and C. Lohmann. 1996a. Orientation and open-sea navigation in sea turtles. Journal of Experimental Biology 199:73-81.

Lohmann, K. J. and C. M. F. Lohmann. 1996b. Detection of magnetic field intensity by sea turtles. Nature 4:5.0.

Lohmann, K. J., J. T. Hester and C. Lohmann. 1999. Long-distance navigation in sea turtles. Ethology, Ecology \& Evolution 11:1-23.

Lohmann, K. J., S. D. Cain, S. A. Dodge and C. M. F. Lohmann. 2001. Regional magnetic fields as navigational markers for sea turtles. Science 294:364-366.

Lohmann, K. J., C. M. F. Lohmann, L. M. Ehrhart, D. A. Bagley and T. Swing. 2004. Geomagnetic map used in sea-turtle navigation. Nature 428:909-910.

Lohmann, K. J., C. M. F. Lohmann and N. F. Putman. 2007. Magnetic maps in animals: Nature's GPS. Journal of Experimental Biology 210:3697-3705.

Lohmann, K. J., C. M. F. Lohmann and C. S. Endres. 2008a. The sensory ecology of ocean navigation. Journal of Experimental Biology 211:1719-1728.

Lohmann, K. J., P. Luschi and G. C. Hays. 2008b. Goal navigation and island-finding in sea turtles. Journal of Experimental Marine Biology and Ecology 356:83-95.

Lohmann, K. J., N. F. Putman and C. M. F. Lohmann. 2011. The magnetic map of hatchling loggerhead sea turtles. Current Opinion in Neurobiology 22:336-342. 
Luschi, P., S. Benhamou, C. Girard, S. Ciccione, D. Roos, J. Sudre and S. Benvenuti. 2007. Marine turtles use geomagnetic cues during open-sea homing. Current Biology 17:126-133.

Marhold, S., W. Wiltschko and H. Burda. 1997. A magnetic polarity compass for direction finding in a subterranean mammal. Naturwissenschaften 84:421-423.

Mate, B., R. Mesecar and B. Lagerquist. 2007. The evolution of satellite-monitored radio tags for large whales: One laboratory's experience. Deep Sea Research Part II: Topical Studies in Oceanography 54:224-247.

Mate, B. R., R. Gisiner and J. Mobley. 1998. Local and migratory movements of Hawaiian humpback whales tracked by satellite telemetry. Canadian Journal of Zoology 76:863.

Muheim, R., F. R. Moore and J. B. Phillips. 2006. Calibration of magnetic and celestial compass cues in migratory birds - a review of cue-conflict experiments. Journal of Experimental Biology 209:2-17.

Norris, K. S. 1967. Some observations on the migration and orientation of marine mammals. Pages 101-125 R. M. Storm ed. in Animal Orientation and Navigation. Corvallis: Oregon State University Press.

Putman, N. F., C. S. Endres, C. M. Lohmann and K. J. Lohmann. 2011. Longitude perception and bicoordinate magnetic maps in sea turtles. Current Biology 21:463-466.

Rasmussen, K., D. M. Palacios, J. Calambokidis, M. T. Saborío, L. Dalla Rosa, E. R. Secchi, G. H. Steiger, J. M. Allen and G. S. Stone. 2007. Southern hemisphere humpback whales wintering off Central America: Insights from water temperature into the longest mammalian migration. Biology Letters 3:302-305.

Stevick, P. T., A. Aguayo, J. M. Allen, I. C. Avila, J. Capella, C. Castro, K. Chater, L. Dalla Rosa, M. H. Engel, F. Felix, L. Florez-Gonzalez, A. Freitas, B. Haase, M. Llano, L. Lodi, E. Munoz, C. Olavarria, E. R. Secchi, M. Scheidat and S. Siciliano. 2004. Migrations of individually identified humpback whales between the Antarctic Peninsula and South America. Journal of Cetacean Research and Management 6:109-113.

Stevick, P. T., M. C. Neves, F. Johansen, M. H. Engel, J. Allen, M. C. C. Marcondes and C. Carlson. 2011. A quarter of a world away: Female humpback whale moves $10,000 \mathrm{~km}$ between breeding areas. Biology Letters 7:299-302.

Stone, G., L. Florez-Gonzalez and S. Katona. 1990. Whale migration record. Nature 346:705-705. 
Vincent, C., B. J. Mcconnell, V. Ridoux and M. A. Fedak. 2002. Assessment of Argos location accuracy from satellite tags deployed on captive gray seals. Marine Mammal Science 18:156-166.

Walker, M. M., J. L. Kirschvink, G. Ahmed and A. E. Dizon. 1992. Evidence that fin whales respond to the geomagnetic field during migration. Journal of Experimental Biology 171:67-78.

Wiltschko, R. and W. Wiltschko. 2003. Avian navigation: From historical to modern concepts. Animal Behaviour 65:257-272.

Wiltschko, W. and R. Wiltschko. 2005. Magnetic orientation and magnetoreception in birds and other animals. Journal of Comparative Physiology. A, Sensory, Neural, and Behavioral Physiology 191:675. 


\section{Chapter 3}

Snapping shrimp sounds as a potential acoustic orientation cue for migrating humpback whales 


\subsection{Introduction}

Most baleen whales undertake long annual migrations during which advanced navigation and orientation mechanisms are essential. The humpback whale (Megaptera novaeangliae) has the best studied migration of any baleen whale species. However, despite extensive speculation on the subject, there is still very little information about what mechanisms and senses these whales utilize to navigate during migration. A number of orientation cues are available to an animal during these long distance movements, but one cue that has not received much attention thus far is sound. In the ocean, sound, particularly low frequency (LF) sound, can travel for tens or even hundreds of kilometers, which makes it well suited as a distance sense (Norris 1967, Payne and McVay 1971). There are many sources of LF sound in the ocean, such as geological activity, waves breaking on a shoreline, or even stable shipping lanes, which all come from predictable enough locations to provide orientation information. Additionally, the vocalizations and hearing capabilities of humpback whales are specialized for the generation and reception of LF sound (Wartzok and Ketten 1999), making it a likely source of environmental information. While it would be extremely interesting to address the role of sound in long distance humpback whale navigation, studying the sensory cues whales use for navigation on this scale is an extremely difficult undertaking.

Fortunately, several populations of humpback whales undertake coastal migrations, a locale which is considerably easier to study. Coastal areas present complex, shallow environments with obstacles, such as rocky reefs or islands and coral reefs, around which a migrating whale must maneuver. Shallow coastal environments are frequently turbid, with vision sometimes limited to just a few meters. At this range, an obstacle could represent a collision hazard to a whale, due to insufficient reaction time. It is therefore unlikely that vision provides sufficient information for a whale to navigate a complicated near-shore environment. On the other hand, sound is transmitted long distances through water with little attenuation. Rocky reefs, coral reefs, and islands are exceptionally noisy locations with numerous acoustic cues, both biotic and abiotic, that can indicate their presence (Montgomery et al. 2006). The noises generated on reefs and 
islands are produced by a combination of reef-associated animals and various abiotic sources (Montgomery et al. 2006). Multiple species of reef fish larvae have been found to use reef sounds to orient towards these sites for settlement (Tolimieri et al. 2000, Simpson et al. 2005), in some cases detecting the reefs $8 \mathrm{~km}$ or more offshore (Radford et al. 2011).

Abiotic ambient noise in the ocean is mainly made up of sounds from wind and breaking waves (Wenz 1962). In the near-shore environment, breaking waves are the dominant source of noise between $100 \mathrm{~Hz}$ and $1 \mathrm{kHz}$, and while breaking surf generates noise up to at least $20 \mathrm{kHz}$, biotic sounds dominate at these higher frequencies (Deane 2000). Noise levels from waves scale with the height of the surface waves squared (Deane 2000). Most of this sound is generated by the oscillation of bubbles created by air entrainment as the wave breaks (Deane 1997). However, there is a very LF component, of a few tens of $\mathrm{Hz}$, that is possibly due to 'surfseisms', which are the elastic waves generated in the seafloor by surf pounding on the shore (Deane 1997). A LF surf beat can often be heard kilometers away from shore (Wilson Jr et al. 1985). The acoustics of waves crashing on islands or reefs is poorly studied, but likely resembles the LF crash of waves on the shore. The impact of waves breaking on a shore or rocky reef could generate loud LF sounds that could serve as a sound beacon for a particular location.

In addition to the noise produced by abiotic processes, fish, snapping shrimp and other invertebrates generate a significant amount of noise in some locations. Some marine species prefer specific depths and environments, and if these animals consistently make noise, they can act as signposts for particular habitats, indicating not only depth, but also ecological factors such as substrate type (Simpson et al. 2005). In some sites, biological sounds can dominate the ambient noise field in certain frequency bands. However, of all the sounds in the ocean produced by animals, none exceeds the prevalence and levels of snapping shrimp (Johnson et al. 1947).

Snapping shrimp are crustaceans that produce a loud snapping sound by an extremely rapid closure of a large snapper claw. This closure produces cavitating 
bubbles, the collapse of which generate an extremely loud snap sound (Versluis et al. 2000). These snaps are broadband, ranging from tens of Hertz to $>200 \mathrm{kHz}$ with a peak to peak source level of 183 to $189 \mathrm{~dB}$ re $1 \mu \mathrm{Pa}$ (Au and Banks 1997). A typical spectrum of a snap has a low frequency peak, between 2 and $5 \mathrm{kHz}$, with a spectral density level of about $120 \mathrm{~dB}$ re $1 \mu \mathrm{Pa}^{2} / \mathrm{Hz}$ in this band (Au and Banks 1997). Snapping shrimp occur in such large numbers in some areas that the combined snapping of the shrimp result in a continuous loud underwater crackle, often compared to the sound of frying fat (Johnson et al. 1947). In some locations, the sound of snapping shrimp is so loud that it exceeds wind-generated noise for sea states up to 7 (Readhead 1997).

Snapping shrimp occur at depths shallower than $55 \mathrm{~m}$ where the water temperature is greater than $11^{\circ} \mathrm{C}$, and on bottoms composed of rock, shell, coral or other sheltering material (Everest et al. 1948). They show a slight diurnal pattern in noise production, with more snaps produced during dusk than any other time of day (Radford et al. 2008). Large variations in noise level have been observed over small distances as bottom conditions change, with the highest noise levels over rocky and coral areas, and the lowest levels where the bottom is mud or sand (Cato and Bell 1992). Due to their prevalence, and their preference for certain depths and substrate types, snapping shrimp could provide reliable acoustic information not only on the location of reefs and other underwater features, but also on the depth and bottom type. Snapping shrimp, as well as other reef sounds, have been shown to aid in the attraction and settlement of some reef fish larvae (Tolimieri et al. 2000, Simpson et al. 2005). Fish larvae may be particularly attracted to sounds of biological origin because they indicate a suitable reef environment (Tolimieri et al. 2000).

In order for a whale to use acoustic cues to detect an obstacle, the strength of the sound signal must exceed the background noise. There is a large amount of background noise in the ocean that can drown out a signal, with the primary contribution coming from wind generated surface waves (Wenz 1962). The levels of this ambient noise correlate well with wind speed and sea state (Knudsen et al. 1948, Wenz 1962, Cato and Tavener 1997, Cato and McCauley 2002, Ma et al. 2005). While this correlation was originally 
found to only hold true for frequencies between $100 \mathrm{~Hz}$ and $10 \mathrm{kHz}$, below which anthropogenic noise predominates, wind-generated noise dominates at frequencies down to $50 \mathrm{~Hz}$ in quieter waters, such as those off the coast of Australia (Cato and McCauley 2002). This frequency range overlaps with the frequency of sounds produced by both snapping shrimp and reef or shore breaking waves. This means that, during high wind conditions, wind generated noise could drown out the signals of an island or reef, making it more difficult for a whale to detect these obstacles at a distance.

The vocalizations and hearing capabilities of baleen whales are specialized for the generation and reception of LF sounds (Wartzok and Ketten 1999). Humpback whales have the best known vocal behavior of the baleen whales, with calls ranging from $20 \mathrm{~Hz}$ all the way up to $24 \mathrm{kHz}$ (Au et al. 2001). Helweg et al. (2000) predict that the best hearing range for humpback whales is between $700 \mathrm{~Hz}$ and $10 \mathrm{kHz}$. This range overlaps with the frequencies that could provide acoustic orientation and obstacle avoidance information. With the whales' highly developed hearing abilities and the acoustic information available from potential obstacles, it would be surprising to find that the whales are not using sound to explore their environment.

The East Australian population of humpback whales are from a relatively well known population of about 7,000 individuals that migrates from feeding grounds in Antarctica to breeding grounds inside the Great Barrier Reef off Queensland, Australia (Noad et al. 2006, Smith et al. 2012). This population has been the focus of a postwhaling population survey since 1978 at Point Lookout on North Stradbroke Island, which is about $50 \mathrm{~km}$ east of Brisbane (Figure 3.1). In this location, the vast majority of this population migrates within $10 \mathrm{~km}$ of the coastline (Noad et al. 2006), in an area with numerous rocky islands. This makes it an ideal location for utilizing cost-effective, shore-based methods to study the mechanisms these whales use to orient around rocky islands. 


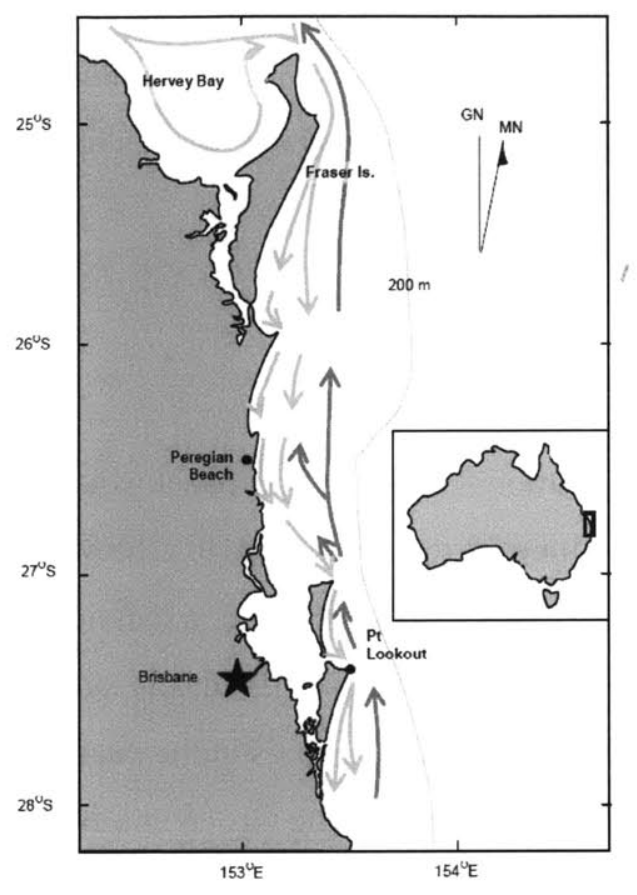

Figure 3.1: The coast of SE Queensland with the migration routes of humpback whales marked. Northward migration route is indicated by the dark grey arrows; southward, by the light grey arrows (Reproduced from Noad et al. 2004).

In 2010, I conducted an observational study of the humpback whales migrating past this coastline, in order to determine if they are using acoustic cues, such as the sounds of snapping shrimp or the crash of breaking waves, to determine the location of a rocky island in their paths. For a whale to acoustically detect a rocky island, the sound signals from the island must exceed the background noise. Due to various factors, the level of a sound signal in water is usually reduced as the range from the source increases. This means that when there are high levels of masking background noise, such as on very windy days, a whale must be closer to the sound source, where the levels are higher, in order to detect the signal above the background sound. If a whale initiates an avoidance reaction when it detects sounds from the island, then the distance of this reaction would be greater during high noise conditions, and smaller during low noise conditions.

However, this reaction most likely has an upper range limit, beyond which an animal may still detect the island, but where a behavioral reaction is unnecessary. Given these 
factors, I utilized hydrophone recordings to determine the detection range of acoustic signals from a rocky island. This was then combined with visual survey techniques to determine the range at which migrating humpback whales reacted to the presence of the island.

\subsection{Methods}

\subsubsection{Study site and data collection}

I conducted an observational study of humpback whales migrating north past the east coast of Australia from June 28 to July 11, 2010. The study site was located at Point Lookout on North Stradbroke Island in Queensland, a location that provided an ideal combination of near-shore islands with high cliffs ideally suited to conducting visual observations. There are two sets of rocky islands in the near-shore waters off Point Lookout that could be used as an obstacle: The Group and Boat Rock (Figure 3.2). Both sets of islands are centered within the visual range of the observation platform, and are within $2 \mathrm{~km}$ of the shore, providing excellent visual coverage of the area. Neither of these islands is ever completely submerged, which means that there is always the possibility for waves to break over the exposed rock. Additionally, both islands are surrounded by a sandy bottom, making them abrupt obstacles in an otherwise graded environment, as well as making it unlikely that snapping shrimp are located anywhere else in the vicinity. Boat Rock was selected as the study obstacle, due to the fact that the water between The Group and shore is very shallow $(<10 \mathrm{~m})$, so very few whale groups migrate through this area. In contrast, a large number of the northern migrating whales pass to either side of Boat Rock, which has an unobstructed approach.

In order to obtain information on the ambient acoustic environment near Boat Rock, a vertical LF four-hydrophone array (SHRU) (Newhall et al. 2007) was deployed from June 28 to July 11, 2010. The SHRU was placed in approximately $24 \mathrm{~m}$ of water, $100 \mathrm{~m}$ to the south of Boat Rock (Figure 3.2) in order to capture the ambient noise field that a whale experiences as it approaches from this direction. The SHRU continuously recorded acoustic data at a sampling rate of $9,765 \mathrm{~Hz}$ on all four hydrophones, with a 
fixed gain of $20 \mathrm{~dB}$ and a sensitivity of $170 \mathrm{~dB}$ re $1 \mathrm{~V} / \mu \mathrm{Pa}$. The flat passband was .452 times the sample rate $(4,424 \mathrm{~Hz})$ and the $-3 \mathrm{~dB}$ point was .49 times the sample rate $(4,785$ $\mathrm{Hz}$ ). Temperature sensors were attached at 2-3 m intervals along the array and sampled the water temperature every $10 \mathrm{~s}$, in order to provide information for acoustic propagation modeling. Additionally, a handheld hydrophone was utilized to obtain recordings at varying distance from Boat Rock, in order to confirm the changes in received level of any sound signal from the rock with distance.

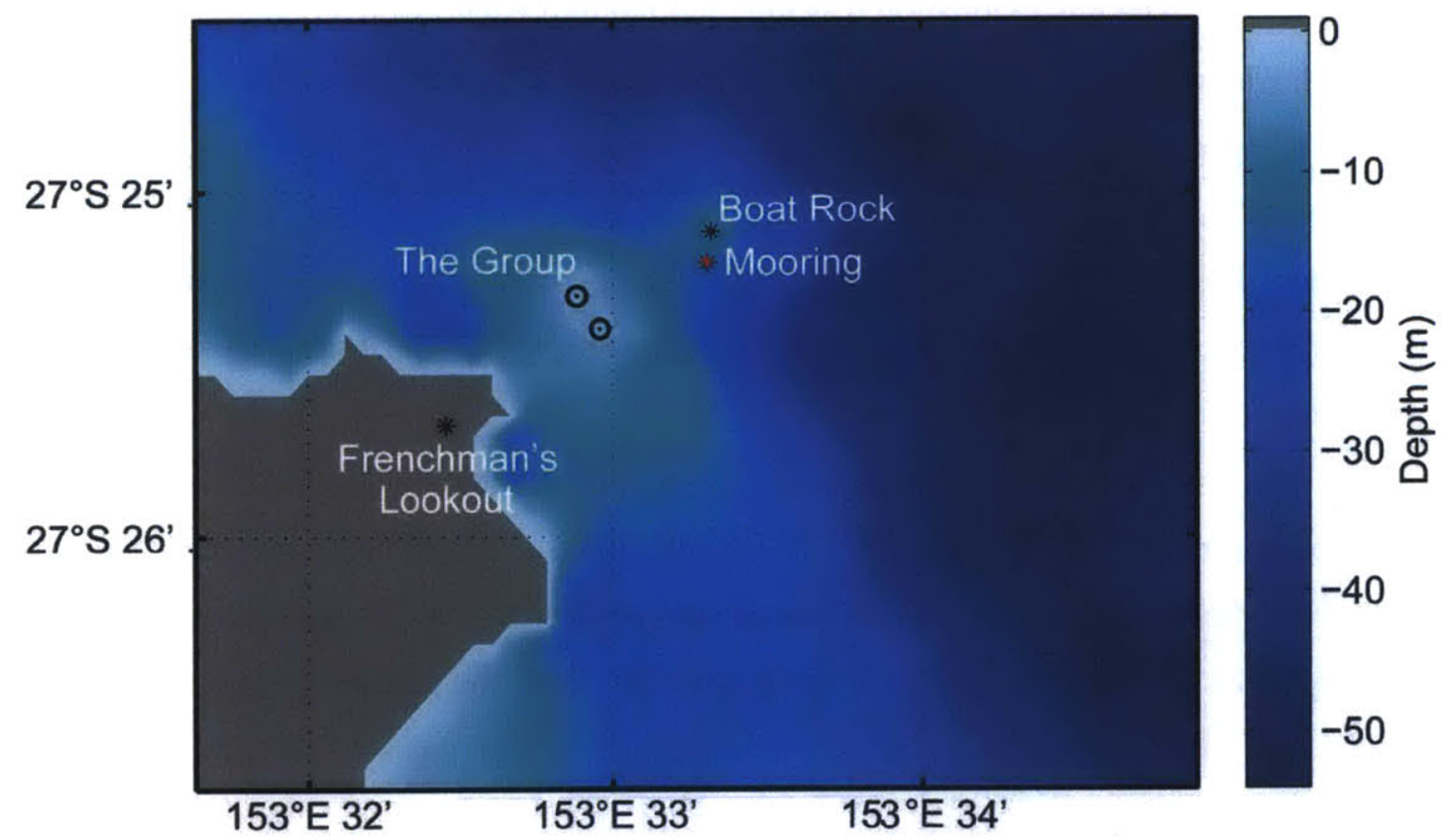

Figure 3.2: A bathymetric map of the study area. The locations of Boat Rock and The Group are marked, as well as the location of the visual survey team at Frenchman's Lookout. The location of the SHRU mooring is marked in red. Bathymetry from Beaman (2010).

In conjunction with these recordings, the field team also visually tracked the whales from shore using a surveyor's theodolite. The visual platform was located on a ridge above Frenchman's Beach $\left(27^{\circ} 25.692^{\prime} \mathrm{S}, 153^{\circ} 32.448^{\prime} \mathrm{E}\right)$, with the theodolite at a height of $58 \mathrm{~m}$ above sea level. This location provided a clear view to approximately 2 $\mathrm{km}$ to the south of Boat Rock, allowing for tracking of the whales as they approached the island. A team of 4-5 observers worked to track the whales as they came within sight. Two to three observers worked as spotters, with each allocated a sector of the ocean to 
scan using bare eyes or $7 \times 50$ binoculars to sight the approaching whales. When whales were spotted, the location was called out to the theodolite operator, who then used the theodolite to locate the whale and send the vertical and horizontal angles of the location directly to a laptop computer. The fifth operator managed the laptop running Cyclops Tracker software, a custom-made marine mammal tracking program (Dr. Eric Kniest, Newcastle University, NSW). The position of the whale group was calculated in Cyclops by using the angles to the sighted location and correcting for tides, curvature of the earth, and refraction. The whale location was then plotted immediately on an on-screen map of the area, where it was time-stamped and assigned a group name. For each group sighted, the laptop operator used Cyclops to record information on: species, group composition, direction of travel, sighting cue (e.g. breach, blow, tail slap etc.), and any other relevant comments. When subsequent theodolite sightings of the same group were input into the computer, Cyclops calculated the group's speed, course and distance from the observation platform.

The visual observers also recorded data on the weather conditions every hour and at the beginning and end of each day. The observers visually estimated and recorded sea state, swell height and direction, wind speed (in knots) and direction, cloud cover, glare, and any other factors affecting visibility (e.g. smoke, haze and squalls).

\subsubsection{Analysis of visual tracks}

The visual data were analyzed in order to determine if there was any change in the movement of the whales around Boat Rock during different acoustic conditions. In order to systematically investigate the features of the whale tracks that approached Boat Rock, I established a grid across the southern approach to the island, similar to that used in Malme et al. (1984). The average bearing at which the whales approached Boat Rock $\left(7^{\circ}\right.$ clockwise from north) was used to establish an approach line, with its origin at Boat Rock. This line extended out to $2 \mathrm{~km}$ to the south, which is the point at which the visibility from the survey site began to diminish. Every half kilometer along the approach line, which is approximately vertical in Figure 3.3, a perpendicular horizontal 
gridline was drawn (Figure 3.3). Then, for each whale group with two or more sightings within $1.5 \mathrm{~km}$ of Boat Rock, the time and location of each horizontal gridline crossing was calculated by interpolation (Figure 3.3). Four variables were then calculated for the time and location of each crossing: the distance of each crossing from the southerly approach line (Dy), the depth at each crossing location $(\mathrm{Db})$, the speed of each group calculated between pairs of gridline crossings $(\mathrm{S})$ and the bearing between pairs of gridline crossings $(\mathrm{Cb})$.

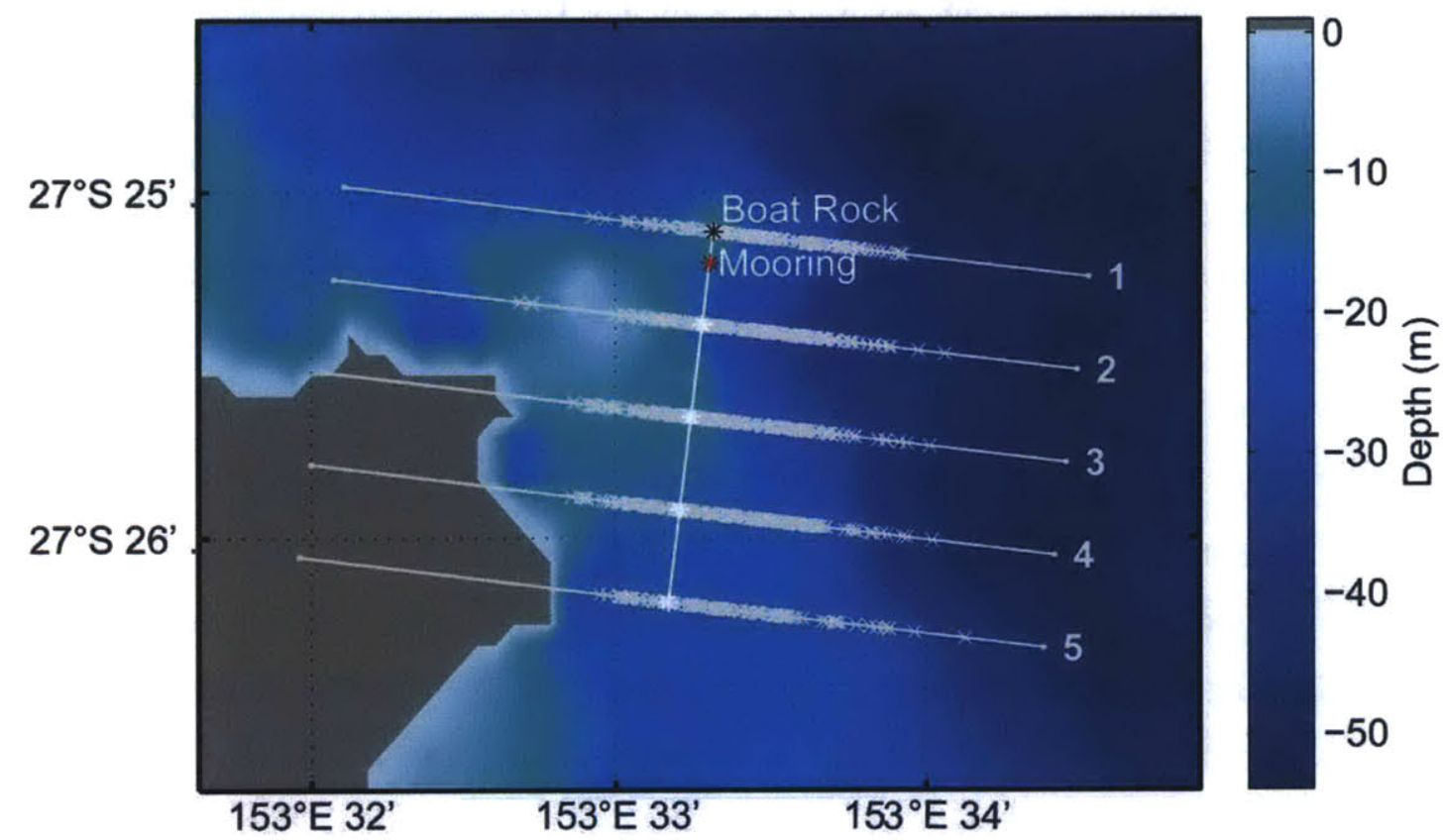

Figure 3.3: A map of the study area with the SHRU mooring location marked in red, and the analysis grid displayed. The approach line starts at Boat Rock and extends south 2 $\mathrm{km}$ along the average heading of all whale tracks ( $\sim 7^{\circ}$ clockwise from north). Perpendicular gridlines are marked every $0.5 \mathrm{~km}$ along the approach line. All locations where whales crossed the horizontal gridlines are marked with x's.

The variables were initially tested for overall differences by comparing the distribution of each variable, at each gridline, to the distribution of the same variable at every other gridline $(1: 2,1: 3,1: 4,1: 5,2: 3,2: 4,2: 5,3: 4,3: 5,4: 5)$. This gives an assessment of whether or not there are any movement patterns underlying the total distribution. A two-sample Cramer-von Mises test was used to compare the distributions 
of Dy, Db and S. This test uses the sum of the squared differences between pairs of distributions to test whether the two samples sets are from the same underlying distribution. A circular Kuiper two-sample test was used to compare the distribution of $\mathrm{Cb}$ between each pair of gridlines. The Kuiper test is a circular analog of the Kolmogorov-Smirnov test, and was chosen because it is invariant under cyclic transformations.

Wind speed and sea state have been shown to correlate well with ambient noise in the ocean (Knudsen et al. 1948, Wenz 1962, Cato and Tavener 1997, Cato and McCauley 2002, Ma et al. 2005), therefore, in this section, wind speed and sea state are used as a proxy for ambient noise levels. The presence of whitecaps was utilized to define the levels of background noise, with no whitecaps present at a sea state of 2.5 or below, and present at sea states above 2.5. Additionally, because the noise from waves breaking correlates with the height of the surface waves squared (Deane 2000), swell height was used as a proxy for levels of the sound signal of waves crashing on Boat Rock. Low swell was defined as waves $1 \mathrm{~m}$ and smaller and high swell as waves greater than $1 \mathrm{~m}$.

In order for a whale to acoustically sense the presence of the island, the sound signal must exceed the background noise. Given this fact, it is assumed here that if a whale is using the sounds of crashing waves on Boat Rock as an orientation cue, then the whale will be able to hear, and respond to this cue from farther away when the swell is large, and the wind and sea state are low, i.e. the signal to noise ratio is high. Conversely, a whale would need to be closer to Boat Rock before it could perceive the sound signal when the swell height was small and the wind and sea state were large, i.e. low signal to noise ratio. Utilizing the variables defined at each gridline, the whales' movement patterns were compared during four different condition combinations:
A. no whitecaps/low swell = low background noise/low sound signal
B. no whitecaps/high swell = low background noise/high sound signal
C. whitecaps/low swell = high background noise/low sound signal
D. whitecaps/high swell $=$ high background noise/high sound signal 
Of these four different conditions, the greatest difference in movement patterns is expected between B. no whitecaps/high swell and C. whitecaps/low swell. Given the above assumptions, a whale would be able to hear the island from farther away during condition B, and therefore would initiate an avoidance reaction from farther away, as evidenced by the four defined variables. Conversely, avoidance of the island is expected to be initiated closer to Boat Rock during condition $\mathrm{C}$, when the signal level is low compared to background noise. Given these assumptions, each of the four variables for each gridline was compared to the same variable for the same gridline during each combination of conditions (A:B, A:C, A:D, B:C, B:D, C:D), utilizing the same tests as above for each variable type.

In order to determine if the range at which the whales initiate an avoidance of Boat Rock changed during different weather conditions, each of the four track variables was compared for each pairwise combination of gridlines $(1: 2,1: 3,1: 4,1: 5,2: 3,2: 4,2: 5$, $3: 4,3: 5,4: 5)$ within each of the four conditions. This indicates whether the whales' movement patterns change with the distance from the rock, and can potentially suggest a distance at which an avoidance reaction is initiated. If any differences in the variables are found, comparing the distances at which these differences occur in each weather condition may give an indication of the range that the whales can detect the rock during each proposed acoustic conditions. This comparison was done within the four given conditions, utilizing the same tests for each variable as stated above.

If the whales are primarily utilizing the sounds of snapping shrimp to localize Boat Rock, then surface breaking waves would serve as the primary source of interfering background noise. Slightly more snaps are produced by snapping shrimp at dusk than any other time of day (Radford et al. 2008), but the levels are steady on average. This means that the sound signal indicating the island's presence is constant, and the detection range of this signal would depend solely on the levels of background noise. To test this possibility, the four variables were compared within gridlines between high sea states and low sea states, as defined above. The same statistical tests of the distributions were used as those stated above. 
While these tests give an initial idea of whether there are any differences present in the visual tracks, conducting multiple comparisons of the same variable in this way increases the likelihood that some fraction of the results will be significant due entirely to chance. There is no universally accepted way to deal with this problem of multiple comparisons, but one approach is to control the family-wise error rate by lowering the alpha level for which a result is considered significant. The most common way to do this is with the Bonferroni correction, in which the alpha level is divided by the number of tests. The grouping of the tests is subjective, but even if the above tests are categorized according to each variable, this gives 85 tests of Dy and Db and 60 tests of S and CB. Adjusting the alpha value according to the number of tests then gives an alpha of 0.00059 . for $\mathrm{Dy}$ and $\mathrm{Db}$ and 0.00083 for $\mathrm{S}$ and $\mathrm{Cb}$.

\subsubsection{Acoustic propagation modeling}

In order to get a better idea of the actual sound field the whales encounter as they approach Boat Rock, the propagation of the sound signal was modeled. For a whale to acoustically detect the island, the power of the sound signal must exceed the background noise; this ratio is known as the signal to noise ratio (SNR). The SNR is calculated as the level of sound received at the whale's location (RL), measured in $\mathrm{dB}$ re $1 \mu \mathrm{Pa}$, minus the noise level (NL). In Australian coastal waters, the primary source of background noise is wind-driven surface waves (Cato and McCauley 2002), with the NL increasing with increasing sea state (Wenz 1962) (Table 3.1). The strength of the sound signal decreases with increasing distance from the source due in part to transmission loss (TL), with the RL equal to the source level (SL) minus TL. Transmission loss is the reduction in the sound energy as the sound spreads geometrically outward from the source. In shallow water, such as this case, the sound spreads out from the source in a cylindrical shape, which means that the energy of the signal is reduced according to

$$
T L=10 \log \left(\frac{r}{r_{r e f}}\right)
$$


where $r$ is the range the sound ray has traveled in meters, and $r_{r e f}$ is set to $1 \mathrm{~m}$.

Aside from the geometrical spreading loss, the other main loss mechanisms of concern are bottom reflection loss (BL), surface scattering loss and volume absorption. Absorption of sound in the ocean is caused by the conversion of sound energy to heat, caused by the shear and volume viscosity of seawater and by ionic relaxation of magnesium sulfate $\left(\mathrm{MgSO}_{4}\right)$ ions in the water (Urick 1984). The volume absorption of sound is frequency dependent, with greater absorption at higher frequencies.

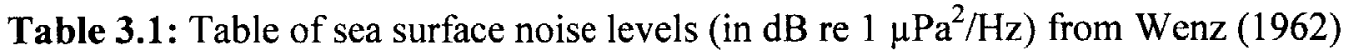
curves at sea states of 0.5 to 5 , utilized in calculations of NL.

\begin{tabular}{|c|c|c|c|c|c|c|}
\hline & \multicolumn{6}{|c|}{ sea state } \\
\hline & 0.5 & 1 & 2 & 3 & 4 & 5 \\
\hline $2 \mathrm{kHz}$ & 40 & 47 & 52 & 54 & 54 & 62 \\
\hline $2.5 \mathrm{kHz}$ & 38 & 46 & 50 & 53 & 53 & 60 \\
\hline $3 \mathrm{kHz}$ & 36 & 45 & 48 & 52 & 52 & 59 \\
\hline $4 \mathrm{kHz}$ & 34 & 42 & 47 & 50 & 50 & 56 \\
\hline $5 \mathrm{kHz}$ & 32 & 40 & 44 & 48 & 48 & 54 \\
\hline
\end{tabular}

In shallow water, if a sound ray leaves the source at a non-horizontal launch angle, it is likely to encounter either the water surface or the ocean bottom and be reflected. These reflections often result in some loss of the acoustic energy, with this loss multiplied by the number of reflections between the source and receiver. For a sound ray encountering the ocean bottom, the amplitude of the reflected sound wave depends on the grazing angle of the incident wave and the density and sound speed of the sediment. There exists a critical grazing angle, $\theta_{\mathrm{c}}$, below which there is perfect reflection of the sound wave:

$$
\cos \left(\theta_{c}\right)=\frac{c_{1}}{c_{2}}
$$

where $c_{1}$ and $c_{2}$ are the sound speeds of the water and sediment, and for which $c_{2}>c_{1}$. However, for a lossy bottom there is no perfect reflection, due to some frequencydependent loss from absorption of the sound wave in the sediment. The ocean bottom often consists of complex layered structures with spatially varying material composition, 
which means that modeling bottom loss (BL) under real ocean conditions is quite complicated.

The surface of the ocean works as both a reflector and a scatterer. If the surface were perfectly smooth, it would form an almost perfect reflector, due to the great difference in sound speed between water and air (Urick 1983). However, the sea surface is never perfectly smooth, and the reflection loss increases with the roughness of the surface, which increases with surface wave height. The reflection loss at the surface can be estimated via simple theory based on the Rayleigh parameter, which is defined as:

$$
R=k \sigma \sin (\theta)
$$

where $k$ is the acoustic wave number $2 \pi / \lambda, \sigma$ is the root mean square of the wave height, and $\theta$ is the grazing angle. When $\mathrm{R}<<1$ the surface acts primarily as a reflector, and when $\mathrm{R}>>1$ the surface acts as a scatterer. The amplitude of the reflection coefficient $\left(V_{c}\right)$ is defined as

$$
V_{c}=V_{0} \exp \left(\frac{-N R^{2}}{2}\right)
$$

where $\mathrm{N}$ is the number of bounces and $\mathrm{V}_{0}$ is the incident wave amplitude, which is set to 1 (Brekhovskikh and Lysanov 2003). This reflection coefficient can then be used to define the loss in $\mathrm{dB}$ as

$$
\text { surface loss }=20 \log \left(\frac{V_{c}}{V_{0}}\right)
$$

At lower frequencies, the loss is smaller due to the fact that the sea is smoother relative to the acoustic wavelength.

Given the above factors, a simplified model of the sound propagation from Boat Rock was created, such that a signal to noise ratio at any given distance could be calculated according to:

$$
S N R=S L-T L-N L-B L-\text { Surface Loss }- \text { Volume absorption }
$$

This equation does not give a precise picture of the sound field near Boat Rock, but rather predicts an average distance at which the whales can detect the island during their 
approach. The SNR at any given distance depends mainly on the initial source level, the number of surface and bottom bounces, and the wind speed at the surface, which influences both the background noise levels and the amount of surface loss.

For this study, only the snapping shrimp sounds were used as an acoustic indicator of the island, as there are no good literature values for the levels of waves crashing on a rocky island and, as will be discussed later, obtaining levels for wave noise from the SHRU proved extremely difficult. These calculations were done utilizing both literature values for the snapping shrimp source levels as well as actual levels based on the received signals at the SHRU. In both cases, the level of sound emitted from Boat Rock is modeled as a point source, which likely underestimates the actual levels produced, as the entire island acts as an extended sound source.

According to laboratory measurements, the snaps of snapping shrimp have a lowfrequency peak between 2 and $5 \mathrm{kHz}$, with an estimated spectral density level of $120 \mathrm{~dB}$ re $1 \mu \mathrm{Pa}^{2} / \mathrm{Hz}$. This level must be adjusted to the bandwidth around each frequency in order to get the band level. The band level is calculated as

$$
\text { Band level }=\text { spectrum level }+10 \log W
$$

where $\mathrm{W}$ is the bandwidth in $\mathrm{Hz}$. Research on hearing suggests that, in mammals, the effective filter bandwidth of the hearing system is roughly $1 / 3$ octave bands (Richardson and Thomson 1998). Therefore, the frequency range of the snapping shrimp was divided into $1 / 3$ octave bands from 2 to $5 \mathrm{kHz}(1800-2250,2250-2800,2800-3550,3550-4450$ and $4450-5650 \mathrm{~Hz}$ ). The source levels adjusted to these bandwidths are then 146, 147, 148,149 , and $150 \mathrm{~dB}$, respectively. The noise levels were estimated for each center frequency from the Wenz ambient noise curves (Wenz 1962) for sea states 0.5 to 5 (Table 3.1), and adjusted by the band level. Transmission spreading loss was calculated according to cylindrical spreading and the total distance traveled by the sound wave. At the frequencies of interest here, loss due to absorption is about $10^{-3} \mathrm{~dB} / \mathrm{m}$, which is relatively negligible, but this loss was still integrated into the model for the given distances. 
The bottom loss was calculated according to a low frequency geoacoustic model by Zhou et al. (2009), which gives an approximation of the seafloor using measured, extrapolated, and predicted values. The most likely sediment composition around Boat Rock consists of fine grain sand. Therefore, the bottom was modeled (courtesy of David Knobles, UT Austin) as fine sand, $50 \mathrm{~m}$ thick, with a sound speed of $1615 \mathrm{~m} / \mathrm{s}$ and a density of $1.7 \mathrm{~g} / \mathrm{cm}^{3}$, which overlay a half space that has a sound speed of $1900 \mathrm{~m} / \mathrm{s}$ and a density of $1.9 \mathrm{~g} / \mathrm{cm}^{3}$. Given a sound speed in the ocean of $1500 \mathrm{~m} / \mathrm{s}$, this means the critical angle for a sound wave reflecting off this bottom would be $22^{\circ}$, below which there would be perfect reflection. As stated above, in the actual marine environment, perfect reflection does not occur as there is always some absorption of the sound in the sediment. This is taken into account in this model, with varying levels of absorption into the sediment based on frequency (Figure 3.4). The actual values utilized for each grazing angle are shown in Table 3.2.

Table 3.2: Bottom loss (dB) for each grazing angle from 2 to $5 \mathrm{kHz}$. Values taken from calculations by Zhou et al. (2009).

\begin{tabular}{|c|c|c|c|c|c|}
\hline & $2 \mathrm{kHz}$ & $2.5 \mathrm{kHz}$ & $3 \mathrm{kHz}$ & $4 \mathrm{kHz}$ & $5 \mathrm{kHz}$ \\
\hline 5 & 1 & 1.5 & 2 & 2.5 & 2.5 \\
\hline 10 & 2 & 3 & 3.5 & 4 & 4 \\
\hline 15 & 3 & 4 & 4.5 & 5 & 5 \\
\hline 20 & 3.5 & 4.5 & 5 & 6 & 6 \\
\hline 25 & 5 & 6 & 7 & 7 & 7 \\
\hline 30 & 7 & 7.25 & 7.5 & 8 & 8 \\
\hline 35 & 8.5 & 8.5 & 8.5 & 8.5 & 8.5 \\
\hline 40 & 9 & 9 & 9 & 9 & 9 \\
\hline 45 & 9.5 & 9.5 & 9.5 & 9.5 & 9.5 \\
\hline 50 & 10 & 10 & 10 & 10 & 10 \\
\hline 55 & 10 & 10 & 10 & 10 & 10 \\
\hline 60 & 10 & 10 & 10 & 10 & 10 \\
\hline 65 & 10.5 & 10.5 & 10.5 & 10.5 & 10.5 \\
\hline 70 & 10.5 & 10.5 & 10.5 & 10.5 & 10.5 \\
\hline 75 & 11 & 11 & 11 & 11 & 11 \\
\hline 80 & 11 & 11 & 11 & 11 & 11 \\
\hline 85 & 11 & 11 & 11 & 11 & 11 \\
\hline 90 & 11 & 11 & 11 & 11 & 11 \\
\hline
\end{tabular}


The surface loss was calculated, according to the equations given above, for all the combinations of 0 to 3 reflections with a sea state of 0.5 to 5 (which corresponds to a peak to peak wave height of 0 to $3 \mathrm{~m}$ ). The surface wave height can be further averaged over the Fresnel zone. The typical wave period for this area of Australia is $10 \mathrm{~s}$, which corresponds to a wavelength of $156 \mathrm{~m}$ for each swell. When this wavelength is taken into account, the Fresnel Zone of a surface wave is $63 \mathrm{~m}$. If the RMS wave height is then integrated over this distance, the height is further reduced. Once this is taken into account, the RMS wave height is very small compared to the wavelengths of the frequencies of interest here $(0.3-0.75 \mathrm{~m})$, and the surface loss becomes negligible. This concurs with measurements using explosive pulses, between 400 and 6,400 Hz, for wave heights of $1-3.5 \mathrm{~m}$ and grazing angles of 10 to $55^{\circ}$, which show a zero $\mathrm{dB}$ reflection loss from the surface (Addlington 1963).

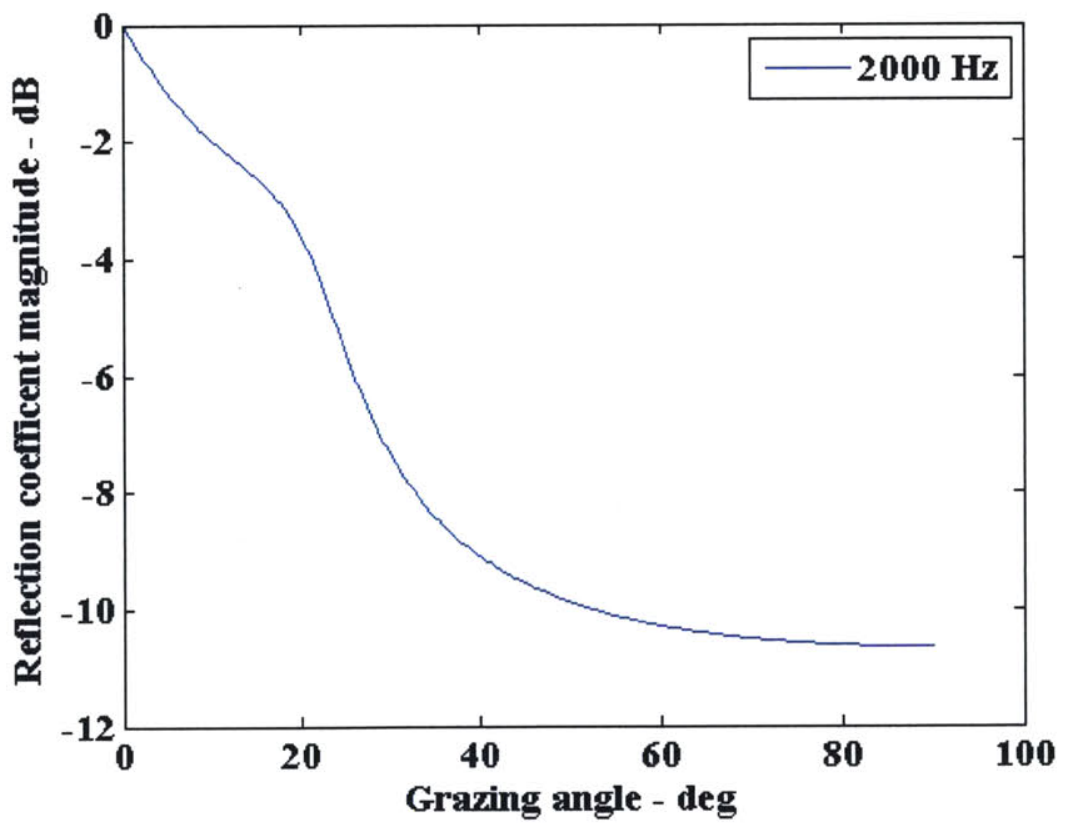

Figure 3.4: An example of the bottom loss versus grazing angle for $2 \mathrm{kHz}$ on fine grain sand. There is a change in the slope at a grazing angle of $22^{\circ}$ (the critical angle), below this the loss is determined by the absorption of the sound into the sediment. (Zhou et al. 2009). 
The above values were used to calculate the SNR for a straight-line ray at a receiver every $10 \mathrm{~m}$ from Boat Rock, out to $2 \mathrm{~km}$, which was the southern range of the visual observations. The water was modeled as an isovelocity column 25 meters deep. The SNR was calculated for each combination of sea states of 0.5 to 5 , and for 0 to 3 bottom and surface reflections, and the resulting SNRs plotted (Figure 3.10, Figure 3.11, Figure 3.12, Figure 3.13). The varying receiver distances had a large influence on the grazing angle of the reflections, as a ray bouncing within the shorter ranges must leave the source at a high angle, and will therefore reflect at a high incident angle, in order to achieve these reflections within the given range. As shown in Table 3.2 and Figure 3.4, these steep grazing angles are associated with bottom loss of about -10 to $-11 \mathrm{~dB} /$ bounce, or -30 to $-33 \mathrm{~dB}$ for 3 bounces. At greater ranges with shallower grazing angles, there is less bottom loss, so the SNR for these distances is higher. Separating the rays into those with direct paths and those with reflections in this way is a simple conceptual method by which to consider the sound field. In actuality, the direct sound path will be present at each of the receiver ranges, and the sound field will be a combination of all ray paths. This is a very simplified approximation of the sound field that the whales experience as they approach Boat Rock, but allows for a first approximation of the levels present.

The threshold at which an animal can detect a signal over the background noise is highly variable among individuals and subject to frequency-dependent interactions. This threshold is particularly difficult to measure in marine mammals, and no measurements exist for baleen whales. A study of the hearing of a harbor porpoise found that the ability of the animal to localize a sound source was reduced when the SNR was less than $10 \mathrm{~dB}$ (Kastelein et al. 2007). However, even when the SNR was as low as $3 \mathrm{~dB}$, the animal still localized the source correctly $79 \%$ of the time (Kastelein et al. 2007). A SNR of 6 $\mathrm{dB}$ was used here as the threshold at which an approaching whale would likely still be able to detect and localize the snapping shrimp signals over the background noise. This level is an approximation, and will vary based on the individual whale and the particular conditions present. 
Using this detection threshold, all of the above sources of loss were then utilized to estimate the distance at which the SNR reached $6 \mathrm{~dB}$, during high noise conditions (sea state 5) and low noise conditions (sea state 0.5 ), for zero and three reflections of the sound ray. This gives an estimate of the minimum and maximum distances at which a whale could detect, and avoid the island.

The same calculations were repeated with the SLs of snapping shrimp from the SHRU recordings. The spectrograms of the recordings from each hydrophone of the SHRU were examined for quality, and the deepest hydrophone, located at approximately $20 \mathrm{~m}$ deep, was chosen for analysis, as it presented the cleanest record. The two weeks of acoustic records from this hydrophone were then loaded in MATLAB, and the root mean square (RMS) levels of the snapping shrimp sounds extracted by a custom made program (courtesy of A. Newhall, WHOI). The mammalian ear is thought to integrate sounds over 200 ms intervals (Plomp and Bouman 1959, Madsen 2005), and therefore the two weeks of acoustic records were split up into $200 \mathrm{~ms}$ intervals. A Fast Fourier Transform of each of these sections was performed, and the frequencies were then filtered to each of the above bandwidths using a Butterworth filter. The highest frequency band, 4450-5650 $\mathrm{Hz}$, was excluded from this section because the upper frequency limit on the SHRU was $4,882 \mathrm{~Hz}$. The filtered waveform was then utilized to calculate the RMS level of the frequency band for each $200 \mathrm{~ms}$ interval. A whale approaching an island would likely be listening for signals over several tens of seconds, and therefore the highest RMS RL for every 20 seconds was determined. These levels were then averaged over the entire two week deployment to obtain mean RMS RLs for each frequency band. The SHRU was located $100 \mathrm{~m}$ to the south of Boat Rock, and assuming a straight-line path between the two, $S L=R L+T L$. Due to the shallow water, spreading was approximated as cylindrical, and TL was calculated as $10 \log \left(\frac{r}{r_{r e f}}\right)$ and the mean SLs were calculated. These estimated SLs were then utilized in the above calculations to estimate the SNR during each of the stated conditions. 
The same RMS calculations as above were conducted for one minute sections of recordings from the handheld hydrophone. This gave relative received levels on the hydrophone at varying distances from Boat Rock $(100,350,600$ and $1100 \mathrm{~m})$. These were then plotted on a $\log$ scale of distances and a least squares line fitted to each frequency band (Figure 3.7). The slope of each of these lines gives an approximation of loss for $\log ($ range) in each frequency band.

\subsection{Results}

\subsubsection{Visual analysis}

Over the course of the two-week survey, 201 tracks of whales migrating north past Point Lookout were recorded (Figure 3.1). Of all groups surveyed, only two passed inshore of The Group. The average heading of the whale groups was $7^{\circ}$ clockwise from magnetic north. The limited visibility to the south of the survey site is apparent in Figure 3.5 , with whales that were migrating farther offshore sighted further to the south. The initial sighting range of those whales migrating close to shore was approximately $2 \mathrm{~km}$ to the south of Boat Rock. Since the view from the observation point was most limited close to shore, $2 \mathrm{~km}$ was chosen as the range for the visual analysis, since most northern migrating whales, regardless of their distance offshore, were spotted before this distance.

The visual observations were limited to daylight hours only, precluding any information on diel patterns of movement around the rock. Additionally, observations were limited to sea states of five or less, when weather did not severely reduce sightability of the whales. The weather observations recorded by the visual team ranged from sea states 1 to 5 , with wind speeds of 3 to 26 knots and swell heights from 0.5 to 2 m. 


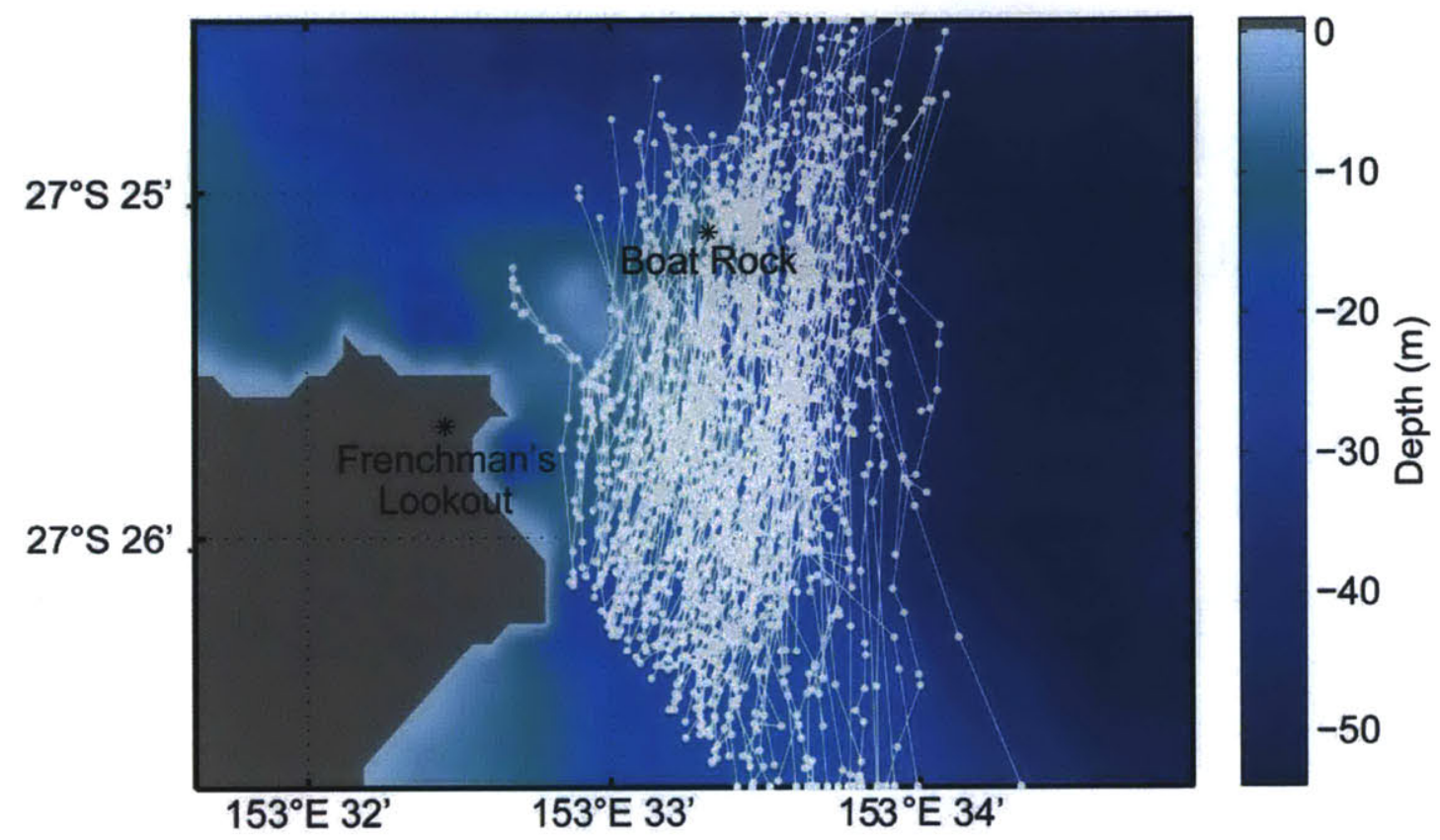

Figure 3.5: Bathymetric map of the study site. All 201 visual tracks recorded during the study period are marked. It can be seen that only 2 groups of whales pass inshore of The Group. The change in initial sighting distance is also apparent, with whales sighted farther south the farther offshore they were.

When the distributions of the variables were compared between each pair of gridlines for the entire dataset, there were no differences found in the distributions of Dy and $\mathrm{S}$ (Table 3.3). There were significant differences between the distributions of $\mathrm{Db}$ at every set of gridlines, which was expected. If the whales were following isobaths as they migrate, then we would expect these distributions to be the same between gridlines. The differences in distributions of depths between sets of gridlines, suggests that the animals do not follow a given depth. There were two differences between the distributions of $\mathrm{Cb}$, between gridline 4 and gridlines 2 and 3 (Table 3.3). Upon closer inspection these differences appeared to be mainly due to a distribution of $\mathrm{Cb}$ at gridline 4 skewed slightly towards higher angles, or more towards the northeast. However, if the Bonferroni adjusted alpha level is used here $(0.00083)$ neither of these results are significant. 
Table 3.3: Pairwise comparison of each variable between gridlines when all the data are analyzed together. If the P-value was greater than 0.05 a zero is displayed, otherwise the $\mathrm{P}$-value is displayed. The Bonferroni adjusted alpha levels are 0.00059 for $\mathrm{Dy}$ and $\mathrm{Db}$ and 0.00083 for $\mathrm{S}$ and $\mathrm{Cb}$.

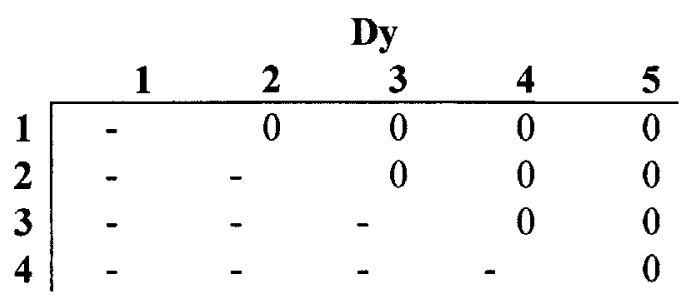

\begin{tabular}{|c|c|c|c|c|}
\hline & \multicolumn{4}{|c|}{$\mathbf{S}$} \\
\hline & 1 & 2 & 3 & 4 \\
\hline 1 & - & 0 & 0 & 0 \\
\hline 2 & - & - & 0 & 0 \\
\hline 3 & - & - & - & 0 \\
\hline
\end{tabular}

\begin{tabular}{r|crrrr}
\multicolumn{7}{c}{} & \multicolumn{1}{c}{ Db } \\
$\mathbf{1}$ & $\mathbf{2}$ & $\mathbf{3}$ & $\mathbf{4}$ & $\mathbf{5}$ \\
\cline { 2 - 6 } $\mathbf{2}$ & - & $<0.01$ & $<0.01$ & $<0.01$ & $<0.01$ \\
$\mathbf{3}$ & - & - & $<0.01$ & $<0.01$ & $<0.01$ \\
$\mathbf{4}$ & - & - & - & $<0.01$ & $<0.01$ \\
& - & - & - & - & $<0.01$
\end{tabular}

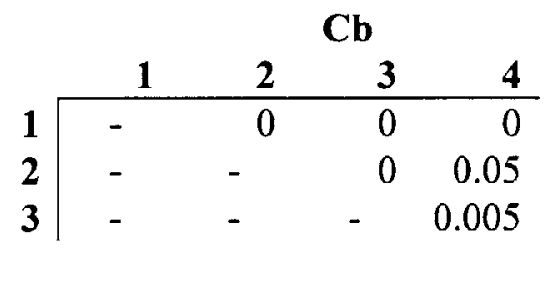

The weather was split into categories $A$ through $\mathrm{D}$, and each of the four variables were compared at the same gridline during each combination of these conditions using either a Cramer-von Mises or circular Kuiper test. The results of these comparisons are shown in Table 3.4. Very few significant differences were found when the track variables were compared across weather conditions. There are two significant differences of the Dy variable, both at the 5th gridline, between No Whitecaps/High Swell and both Whitecaps/High Swell $(\mathrm{P}=0.007)$ and Whitecaps/Low Swell $(\mathrm{P}=0.016)$. Examination of the distribution of Dy during each of these conditions showed a small sample size for the conditions of No Whitecaps/High Swell $(n=12)$. Both of these differences appear to be mainly due to a slightly reduced range of the animals during the No Whitecaps/High Swell conditions, with the whales not found as far offshore as in either of the other conditions. However, when the Bonferroni correction is applied, these difference are no longer significant.

A difference in speed was found for the comparison between No Whitecaps/High Swell and Whitecaps/High Swell at the 3rd $(\mathrm{P}=0.021)$ and 4th $(\mathrm{P}=0.029)$ gridline. The whales traveled slightly slower at the 3 rd gridline when no whitecaps were present. At 
the fourth gridline, during No Whitecaps/High Swell, S had a slightly higher mean, with one large outlier that possibly skewed the mean. The distribution of speeds during Whitecaps/Low Swell extended to speeds much slower (down to $2 \mathrm{~km} / \mathrm{h}$ ) than those seen in the Whitecaps/High Swell conditions. This could indicate some milling about by several of the groups which was frequently observed. Again, when the Bonferroni correction to the alpha level is applied, none of the tests are significant.

Table 3.4: Comparison of the variables between each weather condition combination (A through $\mathrm{D}$ ) at the same gridline. If the P-value was greater than 0.05 a zero is displayed, otherwise the P-value is displayed. The Bonferroni adjusted alpha levels are 0.00059 for $\mathrm{Dy}$ and $\mathrm{Db}$ and 0.00083 for $\mathrm{S}$ and $\mathrm{Cb}$. None of the observed $\mathrm{P}$-values are below this level.

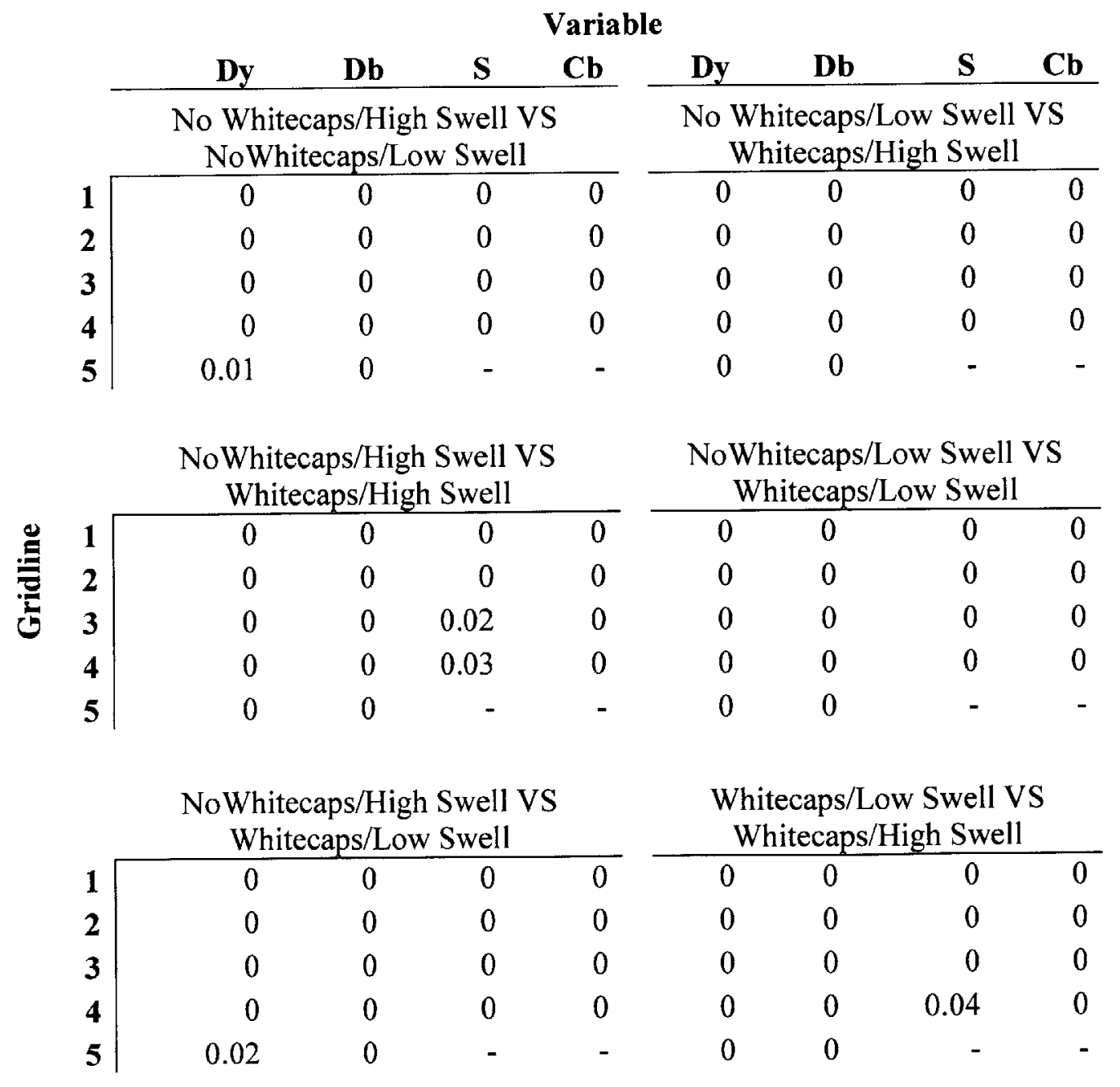


Each gridline was then compared to every other gridline within each set of conditions. The results of the Cramer-von Mises and circular Kuiper tests are shown in Table 3.5. The only significant difference in Dy was between gridlines 1 and 5 during the No Whitecap/High Swell conditions $(\mathrm{P}=0.04)$. The sample size of Dy at gridline 1 was very small $(n=7)$, but there was not a noticeable difference between this distribution and the distribution of Dy at gridline 5. The P-value of the Cramer-von Mises test is less accurate at small sample sizes; therefore this difference may be due to the small number of samples. The only difference in $\mathrm{S}$ is between gridspaces 1 and 4 in No Whitecaps/Low Swell. The whales had a slightly slower mean speed in the 4th gridspace $(4 \mathrm{k} / \mathrm{h})$ than the first $(6 \mathrm{k} / \mathrm{h})$ during these conditions. Additionally, there were significant differences in the course bearings $(\mathrm{Cb})$ during the Whitecaps/Low Swell conditions between gridspaces 1 and 4 and 3 and 4 . These differences appear to be caused by a clustering of slightly higher course bearing angles at gridspace 4. However, none of these P-values are small enough to still be considered significant once the multiple comparisons are taken into account with the Bonferroni correction.

The large number of significant differences in $\mathrm{Db}$ between gridlines is as expected. If the whales were following an isobath, then we would expect them to maintain a certain depth distribution as they approach Boat Rock, and there would be no differences in the gridline distributions. Therefore, it is the gridlines that do not have significant differences between them that are of interest, indicating the possibility that the whales are maintaining their depth between these distances. Gridline 1 showed the least number of significant differences in depth distributions in all weather conditions.

However, the lack of significant differences in the distributions may have been due to the wide range of depths at gridline 1 . This may have limited the ability of the Cramer vonMises test to identify distribution differences.

When the detection range of snapping shrimp was compared during high vs. low noise conditions, none of the variables were found to differ between weather conditions at each gridline (Table 3.6). 
able 3.5: Comparison of each variable between gridlines during each set of weather conditions. If the P-value was greater than $0 . C$ zero is displayed, otherwise the $\mathrm{P}$ value is displayed. The Bonferroni adjusted alpha levels are 0.00059 for Dy and Db and 0.00083 or $\mathrm{S}$ and $\mathrm{Cb}$.

No Whitecaps/Low Swell No Whitecaps/High Swell $\quad$ Whitecaps/Low Swell $\quad$ Whitecaps/High Swell

\begin{tabular}{|c|c|c|c|c|c|c|c|c|c|c|c|c|c|c|c|c|c|c|c|}
\hline \multicolumn{5}{|c|}{ Dy } & \multicolumn{5}{|c|}{ Dy } & \multicolumn{5}{|c|}{ Dy } & \multicolumn{5}{|c|}{ Dy } \\
\hline 1 & 2 & 3 & 4 & 5 & 1 & 2 & 3 & 4 & 5 & 1 & 2 & 3 & 4 & 5 & 1 & 2 & 3 & 4 & 5 \\
\hline & 0 & 0 & 0 & 0 & & 0 & 0 & 0 & 0.04 & & 0 & 0 & 0 & 0 & & 0 & 0 & 0 & 0 \\
\hline & & 0 & 0 & 0 & & & 0 & 0 & 0 & & & 0 & 0 & 0 & & & 0 & 0 & 0 \\
\hline & & & 0 & 0 & & & & 0 & 0 & & & & 0 & 0 & & & & 0 & 0 \\
\hline & & & & 0 & & & & & 0 & & & & & 0 & & & & & 0 \\
\hline
\end{tabular}

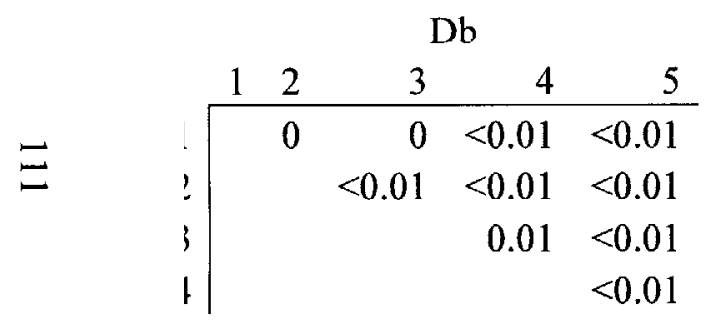

\begin{tabular}{|c|c|c|c|c|}
\hline \multirow[b]{2}{*}{1} & \multicolumn{4}{|c|}{$\mathrm{Db}$} \\
\hline & 2 & 3 & 4 & \\
\hline & 0 & 0 & 0.01 & 0.0 \\
\hline & & 0 & $<0.01$ & 0.03 \\
\hline & & & 0.02 & $<0.01$ \\
\hline
\end{tabular}

\begin{tabular}{|rrrrr}
\multicolumn{5}{c}{$\mathrm{Db}$} \\
1 & 2 & 3 & 4 & 5 \\
\hline & 0 & 0 & 0.01 & $<0.01$ \\
& $<0.01$ & $<0.01$ & $<0.01$ \\
& & $<0.01$ & $<0.01$ \\
& & & & $<0.01$
\end{tabular}
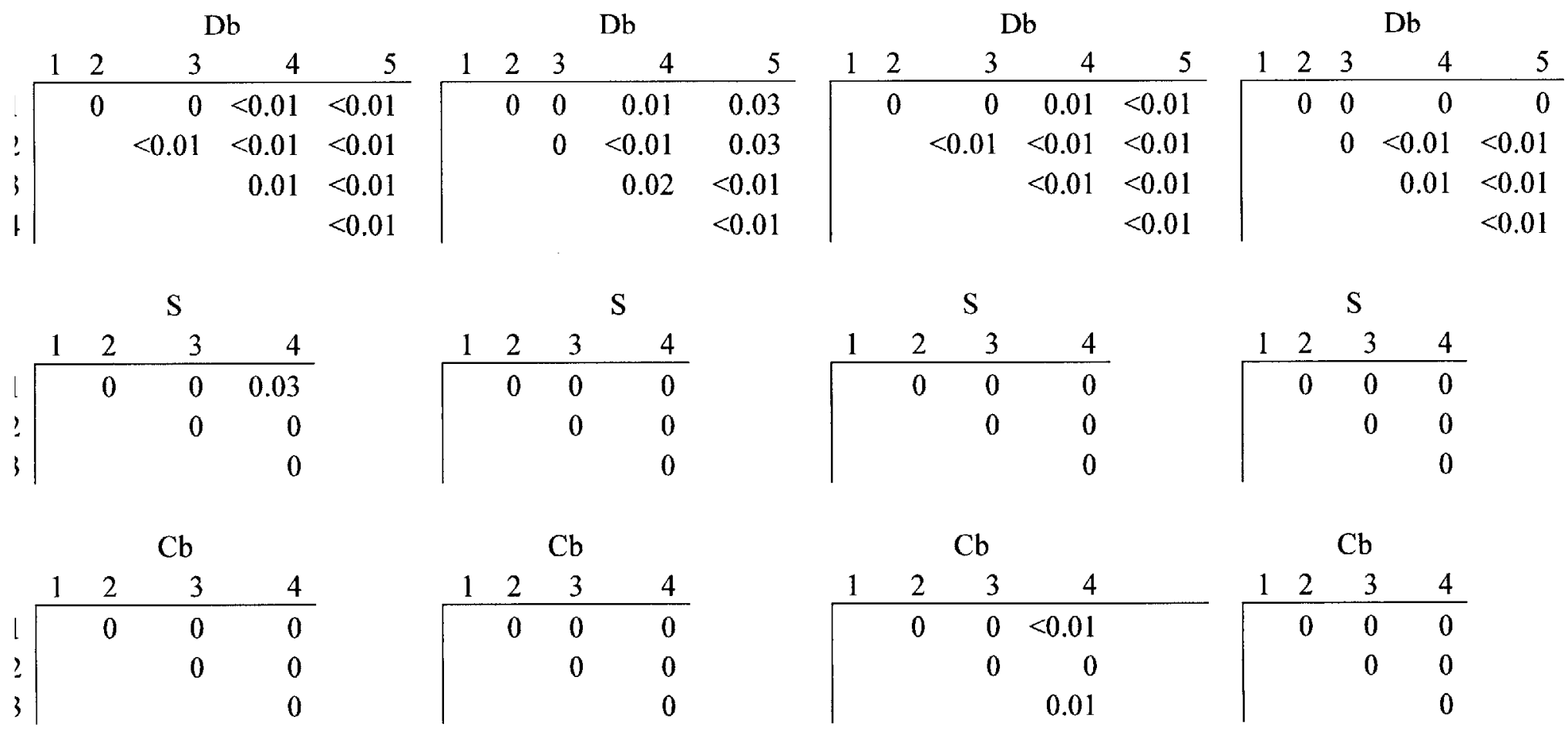

\begin{tabular}{rrrr}
\multicolumn{4}{c}{$\mathrm{Cb}$} \\
1 & 2 & 3 & 4 \\
\hline & 0 & 0 & 0 \\
& & 0 & 0 \\
& & & 0
\end{tabular}

\begin{tabular}{rrrr}
\multicolumn{5}{c}{$\mathrm{Cb}$} \\
1 & 2 & 3 & 4 \\
\hline & 0 & 0 & $<0.01$ \\
& & 0 & 0 \\
& & & 0.01
\end{tabular}

\begin{tabular}{|c|c|c|c|}
\hline \multicolumn{4}{|c|}{$\mathrm{Cb}$} \\
\hline 1 & 2 & 3 & 4 \\
\hline & 0 & 0 & 0 \\
\hline & & 0 & 0 \\
\hline
\end{tabular}


Table 3.6: Comparison of the distribution of each variable at the same gridline during both high $(>2.5)$ and low sea states $(\leq 2.5)$. Zeroes indicate non-significant results.

\begin{tabular}{l|rrrrr}
\multicolumn{5}{c}{} & \multicolumn{5}{c}{ Gridline } \\
\multicolumn{1}{c}{} & 1 & 2 & 3 & 4 & 5 \\
\cline { 2 - 6 } $\mathrm{Dy}$ & 0 & 0 & 0 & 0 & 0 \\
$\mathrm{Db}$ & 0 & 0 & 0 & 0 & 0 \\
$\mathrm{~S}$ & 0 & 0 & 0 & 0 & N/A \\
$\mathrm{Cb}$ & 0 & 0 & 0 & 0 & N/A
\end{tabular}

\subsubsection{Acoustic modeling results}

The SHRU successfully recorded on all four hydrophones for the duration of the deployment. Visual and auditory inspection of the acoustic records revealed clearly evident snapping shrimp sounds, as well as large amounts of humpback song. However, there were no obvious sounds from waves crashing on Boat Rock. There was a regular low frequency signal $(<100 \mathrm{~Hz})$ present in some of the records, with timing elements similar to the swell period. However, the presence of these sounds was not correlated with larger swell height. It was determined that the most likely source of these low frequency sounds was strum noise on the hydrophone array due to strong currents in the area.

The RMS levels recorded on the SHRU in 1/3 octave bands between 2 and $4 \mathrm{kHz}$ over the two week period are displayed in Figure 3.6. Shaded grey areas indicate nighttime, and there is a noticeable peak in levels just after dark and a smaller rise in levels just before dawn. This follows the pattern for snapping shrimp found in the literature, with more snaps produced during dusk than any other time of day (Radford et al. 2008). Additionally, the levels increase slightly in the later portion of the recordings, corresponding with the waning of the moon phase. Radford et al. (2008) noted a peak in snapping shrimp sounds during the new moon. This pattern, combined with the strong and obvious signal from the snapping shrimp in the acoustic records, indicate that the RMS levels are likely due mainly to snapping shrimp. Additionally, the relative levels of the snapping shrimp recorded from the handheld hydrophone were reduced as the distance from Boat Rock increased (Figure 3.7). This indicates that the snapping shrimp sounds are likely coming from shrimp on Boat Rock itself. 


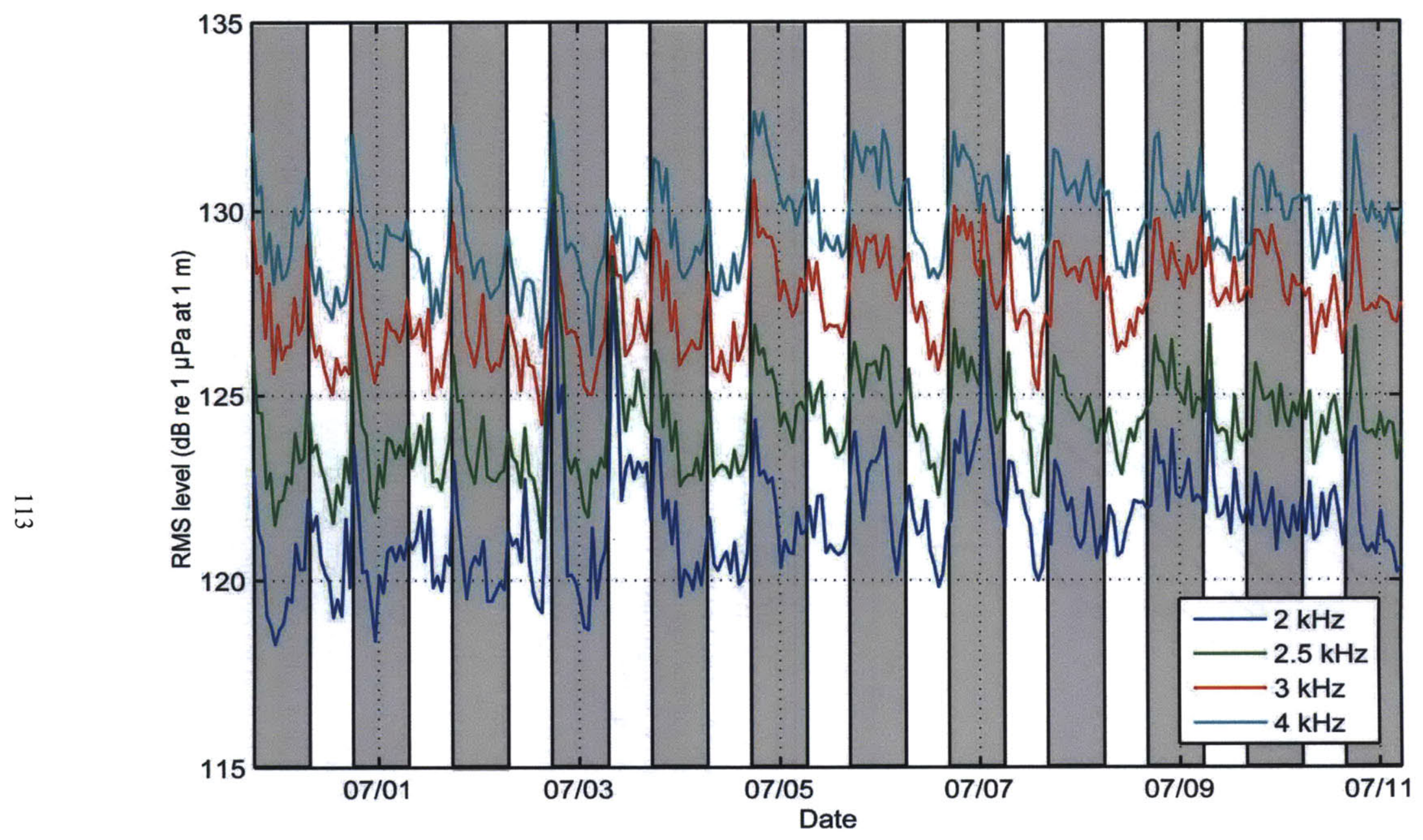

igure 3.6: The RMS received levels at the SHRU for $1 / 3$ octave bands between 2 and $4 \mathrm{kHz}$. The RMS levels were calculated for very $200 \mathrm{~ms}$ interval and the maximum level for every 20 seconds was then averaged over each hour. Each night is marked by the ıaded grey area and there are slightly increased levels just after dusk, with another, smaller peak just before dawn. There is a slight icrease in the overall levels later on in the deployment, as well as a smoothing of the peaks, which corresponds to the waning of the Ioon cycle. 


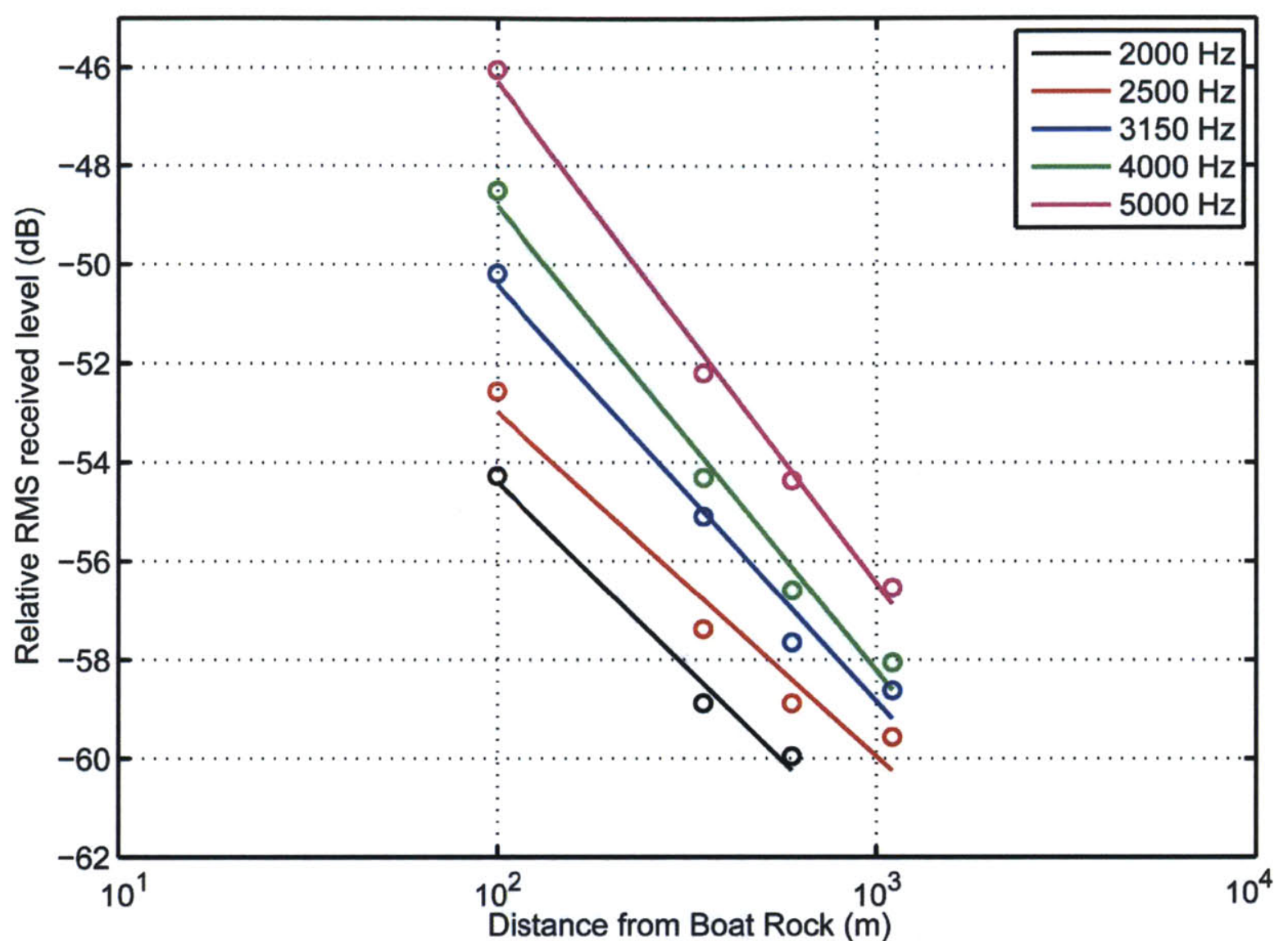

Figure 3.7: The relative RMS received levels on a handheld hydrophone at varying distance from Boat Rock. A least squares regression line was fit to each set of frequency bands and the slope of the line calculated for $\log ($ range). The slopes of each line were: $-7.5,-7.0,-8.4,-9.4$ and $-10.2 \mathrm{~dB}$ for $2,2.5,3,4$ and $5 \mathrm{kHz}$ respectively.

The slopes of the least squares regression lines for the relative received levels on the handheld hydrophone ranged from -7 to $-10 \mathrm{~dB}$ for $\log ($ range). This likely represents the cumulative loss from each frequency for the entirety of the range, indicating that approximating transmission loss as $10 \log \left(\frac{r}{r_{r e f}}\right)$ is appropriate for the given area.

Averaging the levels of each frequency band over the entire two week record gave mean received levels of $121.6,124.2,127.5$ and $129.6 \mathrm{~dB}$ re $1 \mu \mathrm{Pa}$ for 2, 2.5, 3 and $4 \mathrm{kHz}$ respectively. The transmission loss calculated for cylindrical spreading over the $100 \mathrm{~m}$ between Boat Rock and the SHRU was $20 \mathrm{~dB}$. Adding this loss back to the mean received levels gave SLs of 141.6, $144.2,147.5$ and $149.6 \mathrm{~dB}$ re $1 \mu \mathrm{Pa}$ at $1 \mathrm{~m}$. 
The temperature sensors revealed that the water column was relatively well mixed, with a minimum temperature during the study period of $19.5^{\circ} \mathrm{C}$ and a maximum of $22^{\circ} \mathrm{C}$. Given the depths of the sensors, and a standard salinity of 34 PSU, the sound speed of the water ranged from approximately 1520 to $1526 \mathrm{~m} / \mathrm{s}$ (Figure 3.8 ). The water was relatively well mixed at these depths, and the maximum difference in sound speed between the top and bottom temperature sensor was $1.24 \mathrm{~m} / \mathrm{s}$ on July 4 (Figure 3.8 ). When there is a change in sound speed in the water column, a sound ray will curve with a radius $(\mathrm{R})$ such that $R=\frac{c_{0}}{g}$, where $c_{0}$ is the surface sound speed and $g$ is the sound speed gradient (Urick 1983). When $\mathrm{R}$ was calculated for the maximum sound speed difference of $1.24 \mathrm{~m} / \mathrm{s}$ for a $14 \mathrm{~m}$ depth change, the radius of curvature was found to be approximately $17 \mathrm{~km}$. Given this large radius, the rays calculated here can be approximated as their straight-line distances.

The bottom loss was calculated according to the grazing angle values given from the model by Zhou et al. (2009) (Table 3.2). The bottom loss for each receiver distance, for one reflection of the sound ray from the bottom, is presented in Figure 3.9. There is a pattern of initial high bottom loss at receivers close to Boat Rock, due to the high grazing angle required for a sound ray to reflect from the bottom over these short distances. The ray must leave the source at a high angle, and therefore reflect from the bottom at a high angle, in order to achieve one or more reflections from the surface and bottom. When a sound ray reflects from the bottom between Boat Rock and a receiver at any of the greater distances ( $>400 \mathrm{~m})$, the incident angles needed to achieve these reflections are reduced. The loss to the bottom increases with increasing grazing angle, and there is therefore a large amount of bottom loss at the closer distances. This high loss region extends further from Boat Rock when there are more reflections, due to the increased grazing angles required to achieve the increased numbers of reflections. This means that at these distances, the levels from the direct path ray would likely dominate, as they would be significantly greater than those of rays encountering the bottom. The loss is additionally increased with more reflections from the bottom, due to the multiplication of loss by each reflection bounce. 


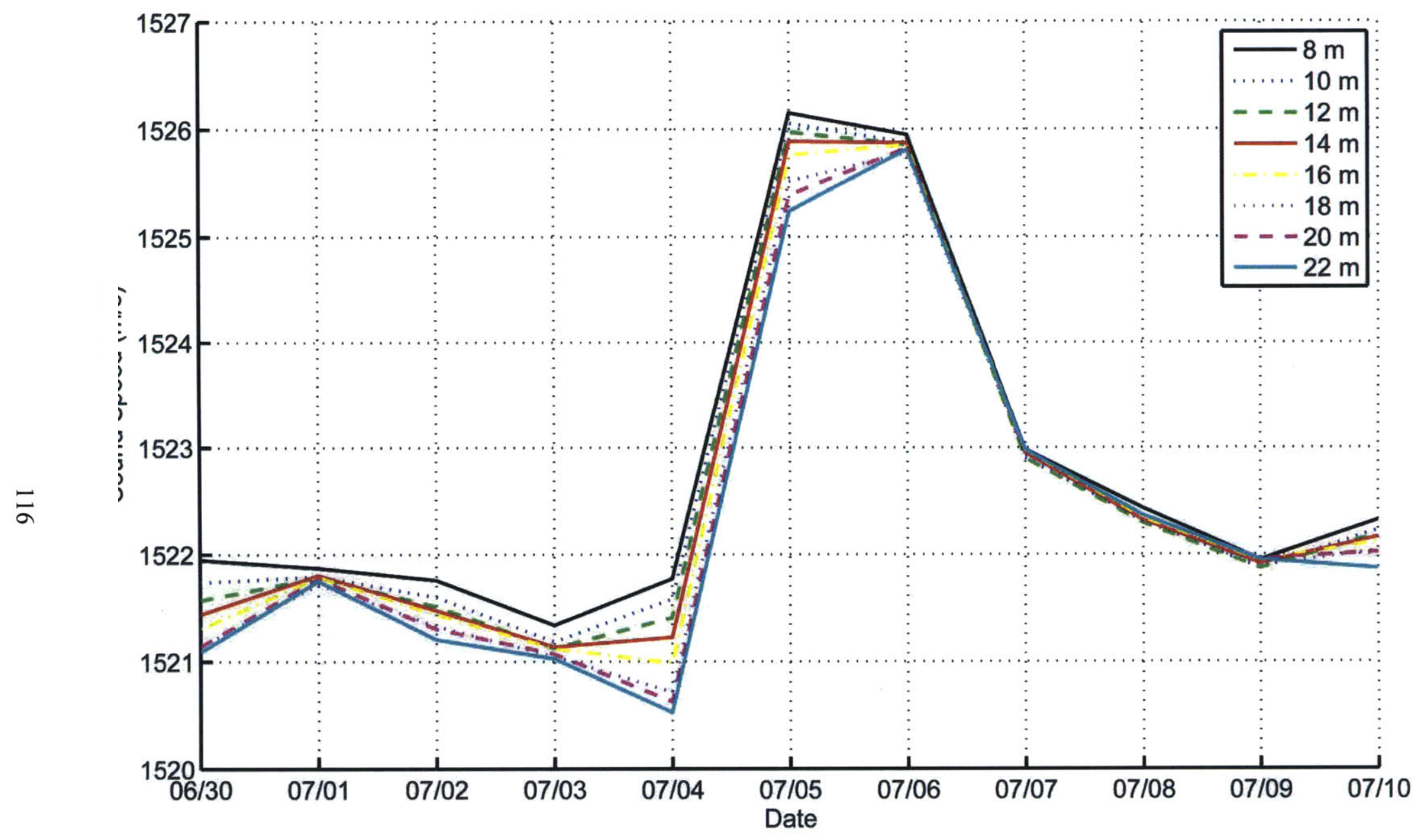

igure 3.8: The daily mean sound speed $(\mathrm{m} / \mathrm{s})$ calculated according to the temperature sensors on the SHRU mooring. The maximur fference between the sound speed at the shallowest temperature sensor $(8 \mathrm{~m})$ and the deepest $(22 \mathrm{~m})$ is $1.24 \mathrm{~m} / \mathrm{s}$ on July 4 . 


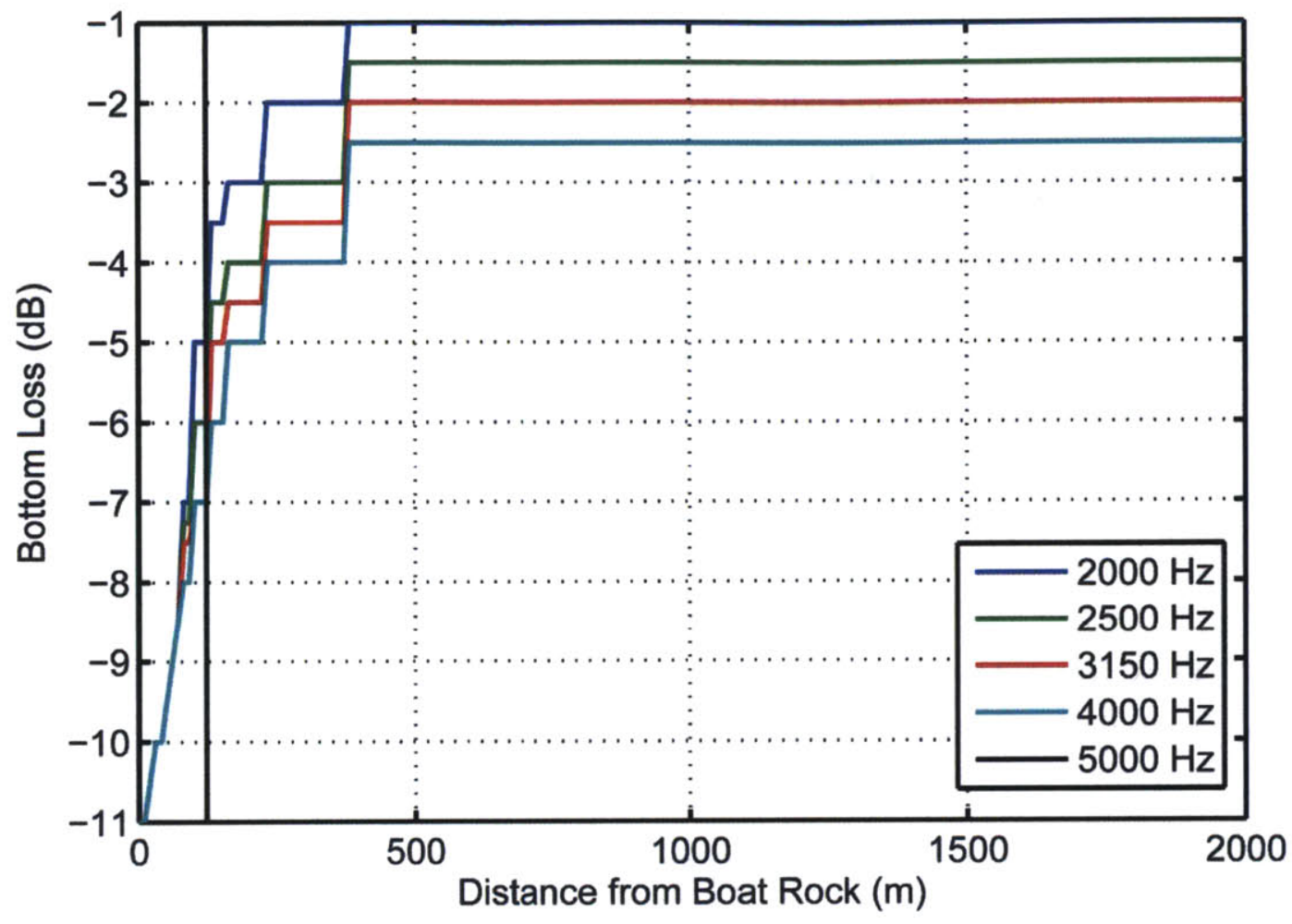

Figure 3.9: Bottom loss for one reflection at a receiver $25 \mathrm{~m}$ deep every $10 \mathrm{~m}$ from Boat Rock out to $2 \mathrm{~km}$. The initial high loss at receivers close to Boat Rock is due to the high grazing angle required for a sound ray to reflect off the bottom in the short distance between the source and receiver. The levels for the direct-path ray will likely dominate at these distances. The distance where the grazing angle falls below the critical angle $\left(22^{\circ}\right)$ is marked with a black line.

The SNRs for all the combinations of noise levels and bounces were calculated, and the four combinations of high and low loss and noise are shown in Figure 3.10, Figure 3.11, Figure 3.12 and Figure 3.13. There are large and obvious differences between the SNRs for zero bounces and those for three bounces. As discussed above, the loss to the bottom is very high at the receivers close to Boat Rock, due to the high grazing angle required for a ray to reflect from the bottom in this range. The high bottom loss at these ranges pushes the SNR to zero in Figure 3.11B. However, since rays spread out from a source in all directions, the sound field will be a combination of all the sound rays present at each receiver distance. Therefore, the rays with a direct path to the receiver 
will dominate at these close receivers and the actual SNR will more closely represent those seen in Figure 3.10 and Figure 3.12.

At the $2 \mathrm{~km}$ visual range of the survey, the loss due to bottom reflections is greatly reduced. In all cases of three bottom reflections, the bottom loss at $2 \mathrm{~km}$ accounts for a $3 \mathrm{~dB}$ reduction in the sound level. This is relatively minimal, and therefore the large differences in SNR seen at $2 \mathrm{~km}$ in all the figures are due almost entirely to the changes in noise level. The maximum SNRs a whale would encounter at $2 \mathrm{~km}$ from Boat Rock are shown in Figure 3.10, and are $47 \mathrm{~dB}$ for the literature values and $42 \mathrm{~dB}$ for the observed values. As expected, the SNR for the literature values is about $5 \mathrm{~dB}$ greater than those for the observed levels, reflecting the difference in SLs. This indicates that during low sea state conditions, the SNR at $2 \mathrm{~km}$ from Boat Rock is likely to be considerably higher than the $6 \mathrm{~dB}$ hearing detection threshold for a whale.

The noise levels at sea state 5 are the highest levels of noise that a whale would have encountered during the visual survey, as observations were not taken in sea states greater than this. The SNRs a whale would have encountered from three reflections of a sound ray from Boat Rock during these conditions are represented in Figure 3.11 and Figure 3.12. Even in the high loss conditions, the minimum SNR at $2 \mathrm{~km}$ is $17 \mathrm{~dB}$ (Figure $3.11 \mathrm{~B}$ ). This is above the estimated $6 \mathrm{~dB}$ detection threshold for the whales, and indicates that they would have likely been able to hear snapping shrimp signals from Boat Rock at distances greater than $2 \mathrm{~km}$ during all the conditions of the survey. 

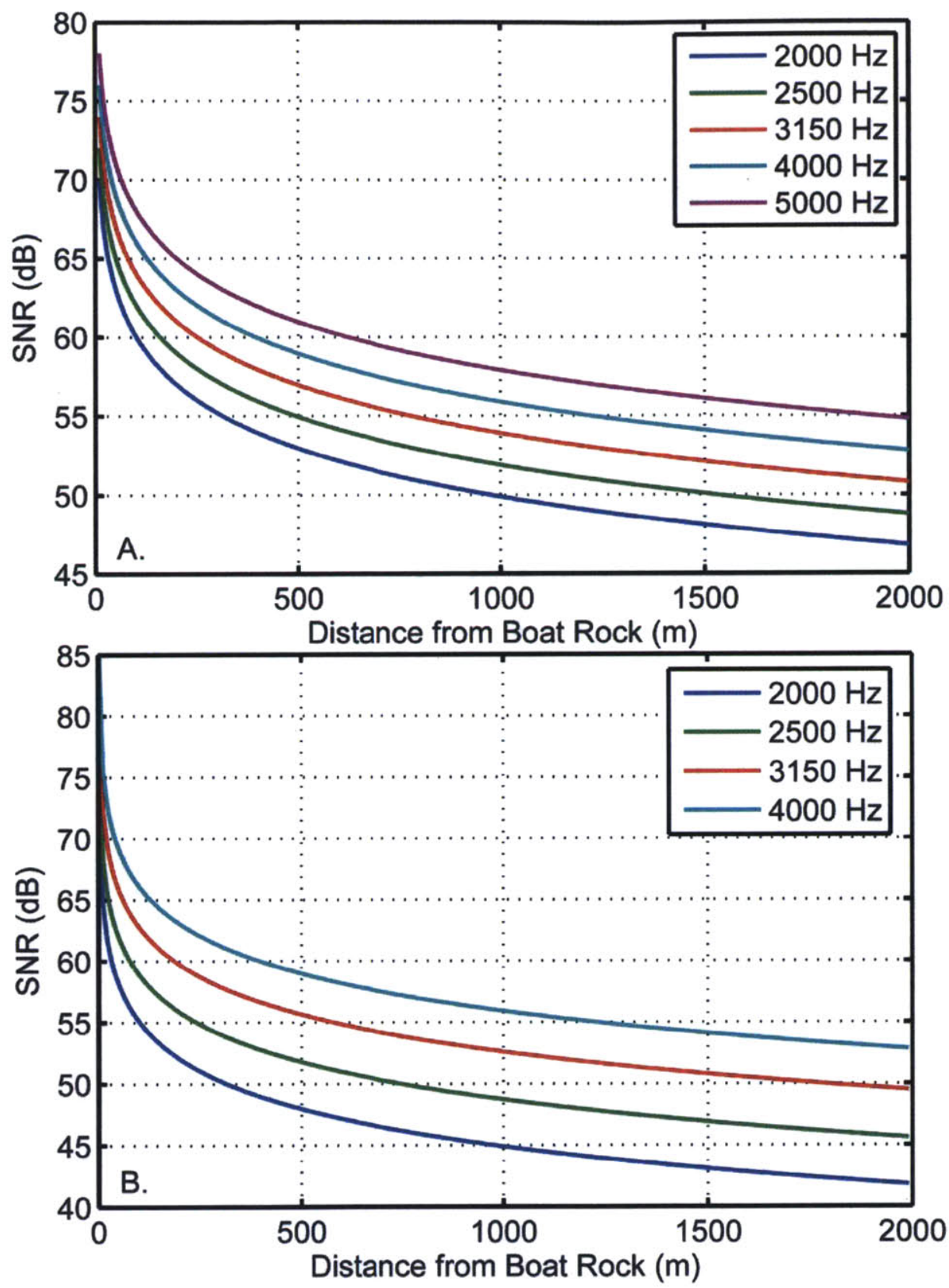

Figure 3.10: The SNR calculated for the direct path (zero bounces) of the sound wave and a sea state of 0.5 . The SNR is calculated every $10 \mathrm{~m}$ from Boat Rock, out to $2 \mathrm{~km}$. The SNRs calculated according to the literature values of snapping shrimp levels are shown in A., and the SNRs calculated according to the levels recorded on the SHRU are shown in B. Panel B only shows frequencies from 2 to $4 \mathrm{kHz}$, as the cutoff frequency for the SHRU was $4,885 \mathrm{~Hz}$. The minimum SNR is seen for $2 \mathrm{kHz}$, at $2 \mathrm{~km}$, and is $47 \mathrm{~dB}$ in A. and $42 \mathrm{~dB}$ in $\mathrm{B}$. 

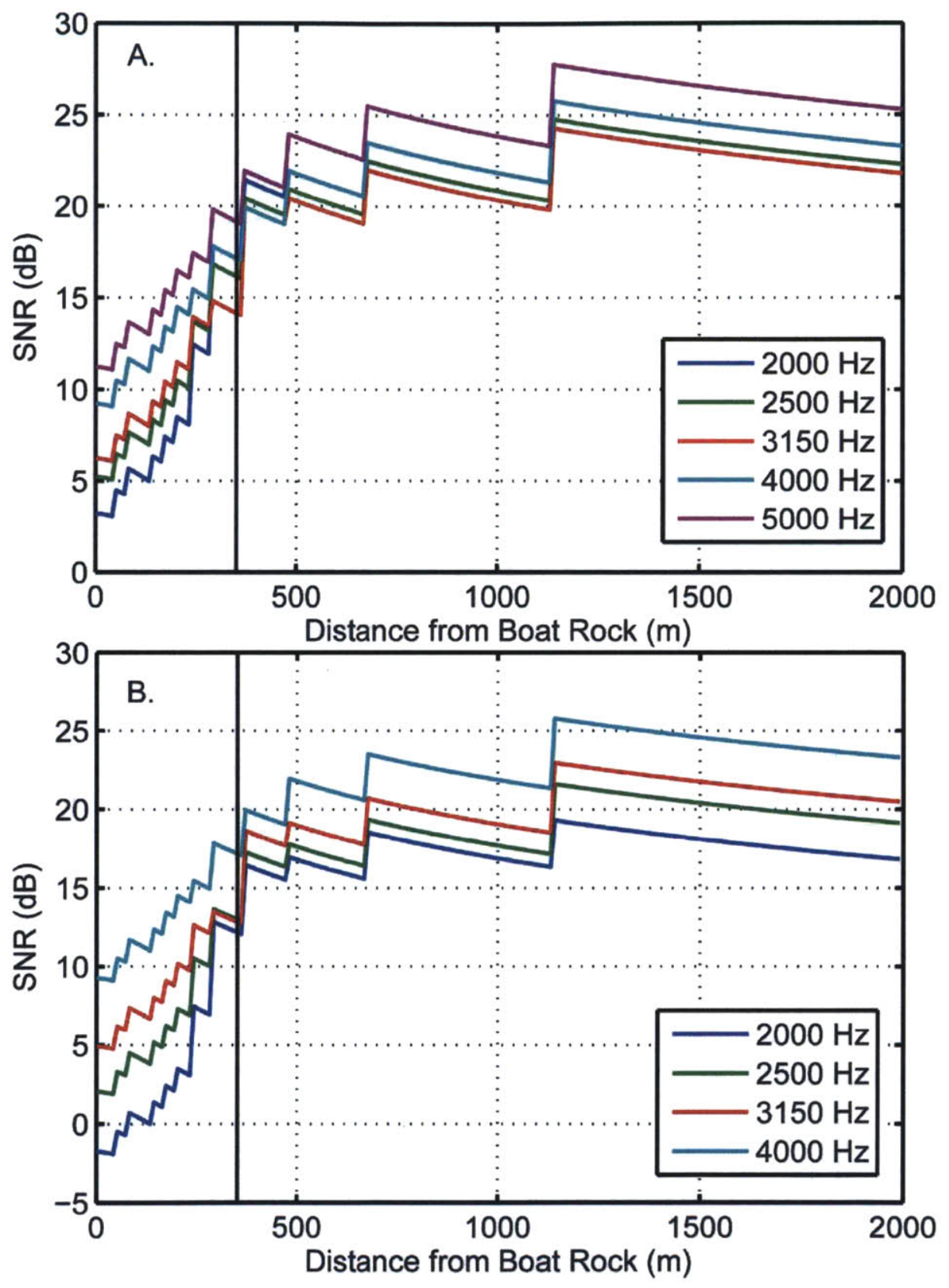

Figure 3.11: The SNR calculated for three reflections of the sound wave from the bottom and a sea state of 5. The SNR is calculated every $10 \mathrm{~m}$ from Boat Rock out to $2 \mathrm{~km}$. The SNRs calculated according to the literature values for snapping shrimp levels are shown in A., and the SNRs calculated according to the levels recorded on the SHRU are shown in B. Panel B only shows frequencies from 2 to $4 \mathrm{kHz}$, as the cutoff frequency for the SHRU was $4,885 \mathrm{~Hz}$. The distance at which the grazing angle goes below the critical angle is marked with a black line. There is an initial high loss predicted close to Boat 
Rock, due to the high grazing angle required for the sound ray to achieve 3 bounces in the short distance. At these ranges, the level for the direct path ray would dominate. The minimum SNR at $2 \mathrm{~km}$ is $22 \mathrm{~dB}$ in $\mathrm{A}$. and $17 \mathrm{~dB}$ in $\mathrm{B}$.
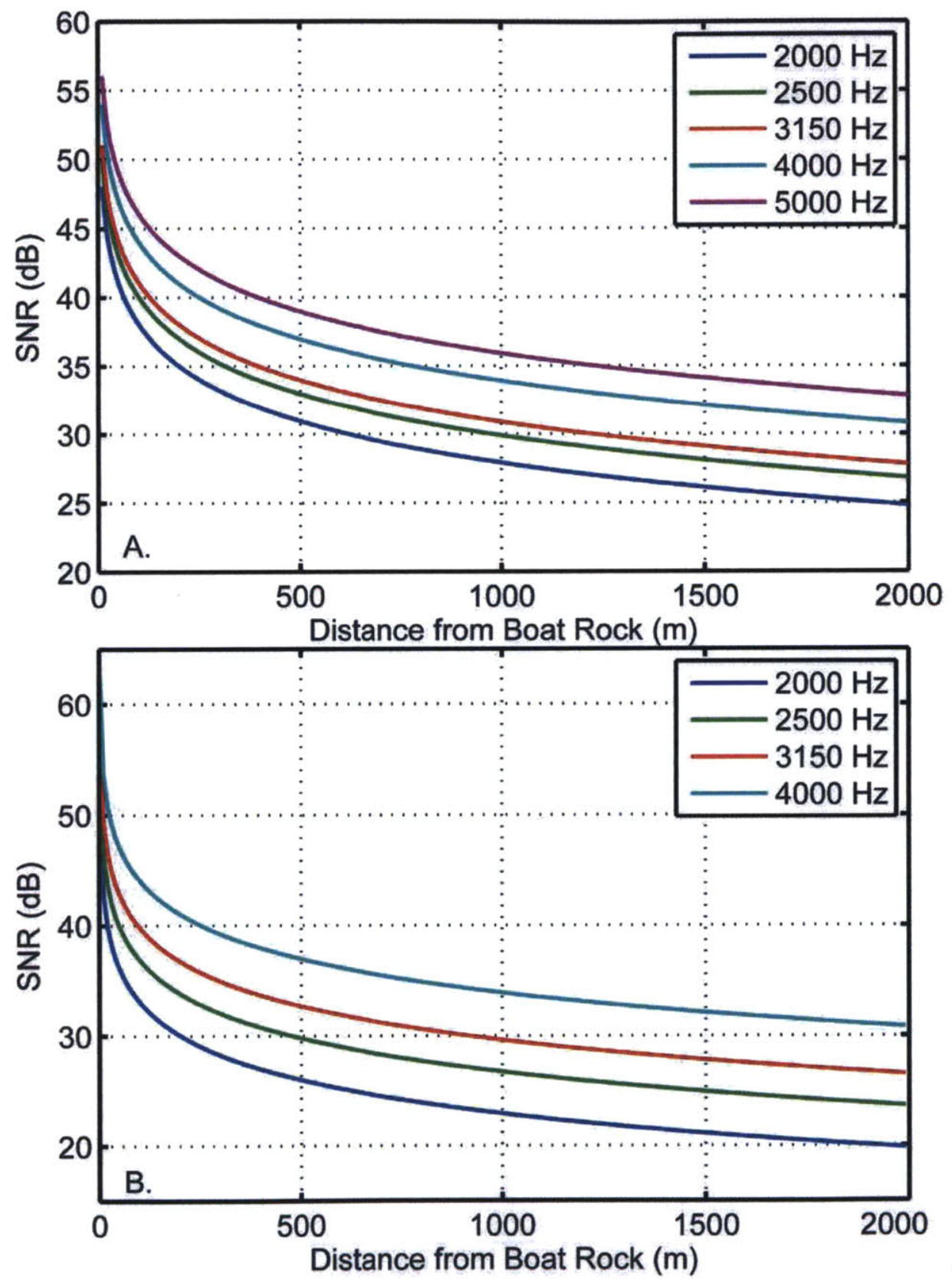

Figure 3.12: The SNR calculated for the direct path (zero bounces) of the sound wave and a sea state of 5. The SNR is calculated every $10 \mathrm{~m}$ from Boat Rock, out to $2 \mathrm{~km}$. The SNRs calculated according to the literature values of snapping shrimp levels are shown in A., and the SNRs calculated according to the levels recorded on the SHRU are 
shown in B. Panel B only shows frequencies from 2 to $4 \mathrm{kHz}$, as the cutoff frequency for the SHRU was $4,885 \mathrm{~Hz}$. The minimum SNR is seen for $2 \mathrm{kHz}$, at $2 \mathrm{~km}$, and is $25 \mathrm{~dB}$ in A. and $20 \mathrm{~dB}$ in $\mathrm{B}$.
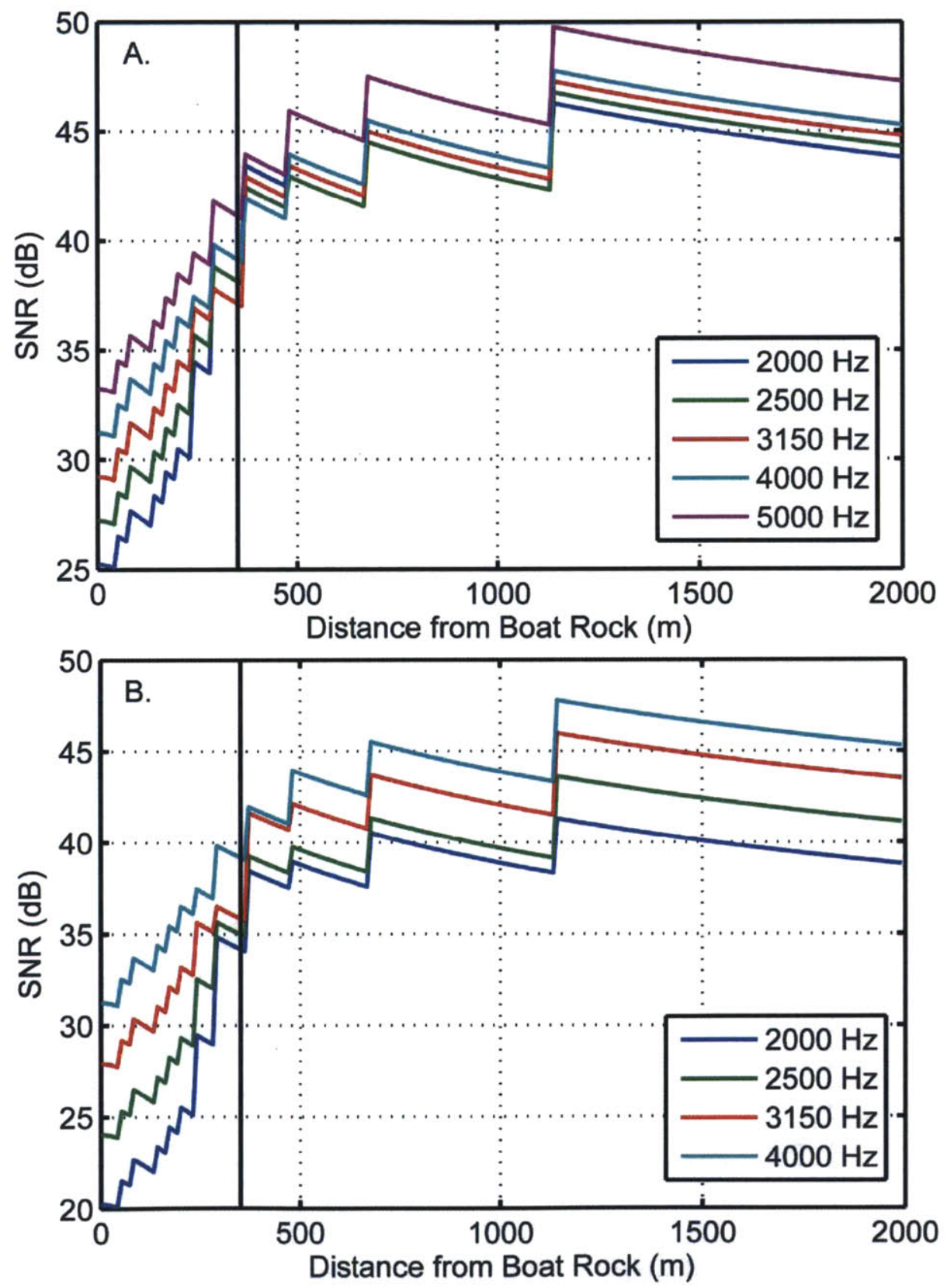

Figure 3.13: The SNR calculated for three reflections of the sound wave from the bottom and a sea state of 0.5 . The SNR is calculated every $10 \mathrm{~m}$ from Boat Rock, out to $2 \mathrm{~km}$. 
The SNRs calculated according to the literature values for snapping shrimp levels are shown in A., and the SNRs calculated according to the levels recorded on the SHRU are shown in B. Panel B only shows frequencies from 2 to $4 \mathrm{kHz}$, as the cutoff frequency for the SHRU was $4,885 \mathrm{~Hz}$. The distance at which the grazing angle goes below the critical angle is marked with a black line. There is an initial high loss predicted close to Boat Rock, due to the high grazing angle required for the sound ray to achieve 3 bounces in the short distance. At these ranges, the level for the direct path ray would dominate. The minimum SNR at $2 \mathrm{~km}$ is $44 \mathrm{~dB}$ in $\mathrm{A}$. and $38 \mathrm{~dB}$ in $\mathrm{B}$.

The maximum distance the snapping shrimp signals from Boat Rock could be heard was estimated for both the literature and observed SLs under both low loss and noise conditions (zero bounces, sea state of 0.5 ) and high loss and noise conditions ( 3 bounces, sea state of 5). The distance at which the SNR reached the estimated detection threshold of $6 \mathrm{~dB}$ for the literature SLs for snapping shrimp, along a ray path with zero bounces, was a maximum of approximately $208 \mathrm{~km}$ during low noise conditions, and a minimum of $35 \mathrm{~km}$ during high loss and high noise conditions. Alternatively, the SNR reached $6 \mathrm{~dB}$ at $8.3 \mathrm{~km}$ during high loss and noise conditions and $136 \mathrm{~km}$ during low loss and noise conditions for the observed snapping shrimp levels. Therefore, the minimum range a whale would have been able to detect snapping shrimp signals from Boat Rock, under the conditions of this model, was $8.3 \mathrm{~km}$, a range greater than that of the visual observations.

\subsection{Discussion}

\subsubsection{Visual observations}

The visual tracks of humpback whales migrating north past Point Lookout, Australia, were collected in order to elucidate whether or not there are any changes in the approach of the whales to Boat Rock that might indicate their use of acoustic signals to detect the island. An assumption behind this study design was that if the approaching whales were using an acoustic signal, either the sounds of crashing waves or snapping shrimp, to detect the island, then they would be expected to initiate a discernible avoidance reaction at the point when they acoustically detected the island. The distance at which this avoidance behavior was initiated would then be determined by the sound 
field conditions, which would influence the point at which the whales could detect the island. However, analysis of the 201 collected visual tracks, using methods similar to those used by Malme et al. (1984) to investigate the effects of noise from the petroleum industry on gray whale behavior, found no consistent changes in the whales' behavior during different proposed sound conditions.

An initial analysis of the visual tracks, comparing each of the four variables to the same variable at every other gridline, tested for any systematic differences in the whale tracks as they approached Boat Rock. Any underlying differences in the variables at each gridline would indicate a difference in the whales' approach behavior, which could be fueled by the topographical and bathymetric differences present at each gridline. This comparison revealed that the only difference between the gridlines was in the course bearing $(\mathrm{Cb})$ between gridline space 4 and gridline spaces 2 and 3 . The course bearings at gridline space 4 clustered around higher positive angles. This is not an unexpected result, as the whales migrate up a relatively straight coastline before they reach Point Lookout (Figure 3.1), and this headland acts as a funnel for whales that migrate close to shore. This means that the whales migrating close to shore as they approach Point Lookout are then forced to take a bearing to the north-east in order to avoid the point. This increased bearing may still be reflected in gridline space 4, which would account for the distribution of slightly higher $\mathrm{Cb}$ bearings for this section.

When the variables were split up according to the combinations of weather conditions, assumed to represent different sound conditions, and compared to the same variable at the same gridline during these different conditions, very few differences were found (Table 3.4). This test was used to determine if there were any changes in the behavior of the whales during the different assumed sound fields that could indicate an increased or decreased avoidance distance. The most telling differences would have been changes in any of the variables between conditions B (no whitecaps/high swell) and C (whitecaps/low swell). However, the only difference found between these conditions was for Dy at gridline 5, which appeared to be due to a slightly reduced distribution of the animals around the approach line during the No Whitecaps/High Swell conditions. While 
it is possible that this difference resulted from a change in the detection range of Boat Rock, it only shows up at this location and in this variable and therefore could equally be due to changes in the acoustics around the headland of Point Lookout during these conditions.

The only other differences found between conditions were slight changes in speed between Whitecaps/High Swell and both No Whitecaps/High Swell and Whitecaps/Low Swell. The whales traveled slightly faster during the Whitecaps/High Swell conditions than in either of the others. These differences could be due to a change in behavior stemming from variations in the detection range of Boat Rock, but this is unlikely, as they do not follow consistent patterns according to the predictions based on the expected differences in sound fields.

The test for changes in each variable between pairs of gridlines, within each set of conditions, was designed to indicate if there was any variations in avoidance distances as the whales approached Boat Rock. These tests also revealed very few significant differences (Table 3.5). The similarities between the depth distribution of the whales at gridline 1 and many of the other gridlines, during all weather conditions, could be due to the wider distribution of depths at the first gridline. This increased distribution makes it more difficult to discern if there are any differences between this and the narrower distributions that may be present at the earlier gridlines, and therefore the Cramer vonMises test may not have detected any differences present in the depth distribution. Additionally, the difference between the course bearing $(\mathrm{Cb})$ between gridline space 4 and both gridline spaces 1 and 3, during the No Whitecaps/High Swell conditions (Table 3.5 ) is due to a higher concentration of the Cbs at higher bearing angles for gridspace 4. This is the same pattern seen in the non-divided data and may be a residual of this effect. Despite these small differences seen in some of the variables, during some of the conditions, there are no consistent patterns found in this analysis.

When the acoustic signal coming from Boat Rock was assumed to be emitted from snapping shrimp, the detection range would be solely determined by the levels of the snapping shrimp in relation to the levels of the background noise. When the four 
variables were compared at the same gridline during either high or low wind conditions, there were no differences found between the distributions (Table 3.6).

The visual data analysis looked at each individual comparison of each of the variables. While this gives an initial idea of whether there are any differences present, when multiple comparisons are conducted, using the same variables, there is an increased likelihood that some fraction of the results will be false positives. When a Bonferonni corrected alpha value was used for each variable $(0.00059$ for $\mathrm{Dy}$ and $\mathrm{Db}$, and 0.00083 for $\mathrm{S}$ and $\mathrm{Cb}$ ) the number of significant results would be considerably reduced.

Therefore it is important to look for patterns of significance in the data as a whole in order to determine if there are any consistent patterns in the movements of the whales that might indicate an avoidance of Boat Rock. There do not appear to be any patterns in the visual data that would indicate the initiation of avoidance to the south of Boat Rock during any of the conditions. This did not follow the prediction and could either indicate that the whales are not using acoustic cues to detect the island, or that there is some underlying difference between the predicted sound field according to the weather conditions and the actual sound field presented to the approaching whales.

\subsubsection{Sound field modeling of Boat Rock}

In order to determine if there was a difference between the predicted and actual sound fields, the sound signals from Boat Rock were modeled during different noise conditions. The initial research plan consisted of utilizing frequencies and source levels for waves crashing on Boat Rock as the acoustic signal for the island. However, literature values for the frequency and levels of the sounds of waves crashing on rock are surprisingly lacking. Additionally, the recordings from the SHRU proved to have no obvious signal that could be attributed to waves breaking on Boat Rock. This could either be due to lack of a strong signal from these crashing waves, or masking of the signal by the strum noise on the hydrophones. However, the sounds of snapping shrimp have been well documented in the literature and presented a strong and consistent signal 
in all the SHRU recordings. Additionally, snapping shrimp have been shown to cluster on hard substrates, such as rocky islands. Therefore, the model calculations were conducted using literature and observed source levels for snapping shrimp as the sound signal of Boat Rock.

The snaps of snapping shrimp were detected at all times during the SHRU deployment, with a slight peak just after nightfall (Figure 3.6). This is consistent with the findings of other studies showing a slight diurnal pattern, with peaks at dusk (Radford et al. 2008). Additionally, recordings from a handheld hydrophone at increasing distances to the south of Boat Rock confirmed that the levels of the snapping shrimp sounds reduced as the distance increased (Figure 3.7). This indicates that the snapping shrimp were located on the rocky island, which follows the findings of studies showing that they prefer a hard substrate (Cato and Bell 1992). When the transmission loss was added back to the averaged RMS received levels for each frequency band, the resulting source levels were between 0 and $5 \mathrm{~dB}$ lower than the levels predicted using the laboratory measurements of Au and Banks (1997). This is a very close match, and indicates that the calculated levels are likely relatively accurate.

When both the literature and observed values for the snapping shrimp levels were used to calculate the SNRs at distances out to $2 \mathrm{~km}$ from Boat Rock, the difference between the SNRs for the high loss and noise and low loss and noise conditions at $2 \mathrm{kHz}$ was $25 \mathrm{~dB}$. These differences between the high loss and low loss conditions were driven by the higher loss due to increased numbers of bounces from the bottom, greater transmission distance, and increased noise levels.

The bottom loss predicted for this area proved to be greatest in regions close to Boat Rock (Figure 3.9). This high loss is due entirely to the high grazing angles required for a ray to achieve reflections from the bottom during these short distances. However, at these close distances, there are likely direct sound rays from the island that would reach the receiver, and therefore the SNR would not reach 0 at the close range predicted in Figure $3.11 \mathrm{~B}$. The sound field would more likely reflect the direct path levels seen in Figure 3.12 B. When the sound wave traveled to receivers at greater distances, such as 
the limit of the range at $2 \mathrm{~km}$, the grazing angles of the bounces are low enough that the influence of loss to the bottom became quite small $(3 \mathrm{~dB})$. This small influence of bottom loss at the limit of the model range $(2 \mathrm{~km})$ means that the main sources of loss were due to geometric spreading loss, which is fairly consistent among the different conditions.

The transmission loss in this model is calculated as a function of the total distance traveled by the sound wave. This distance is slightly greater for sound waves reflecting from the bottom. However, this increased distance is small enough that the increase in TL is minor. Therefore, the main cause of the reduced SNRs at $2 \mathrm{~km}$ in Figure 3.11 and Figure 3.12, over those in Figure 3.10 and Figure 3.13 are the increased noise levels. However, despite the high levels of loss seen in Figure 3.11, in no case does the SNR reach the chosen detection threshold of $6 \mathrm{~dB}$ at $2 \mathrm{~km}$ from Boat Rock. When the limit of the detection range of the snapping shrimp sounds from Boat Rock was calculated for the detection threshold, the minimum detectable distance among all conditions and source levels was found to be $8.3 \mathrm{~km}$. This indicates that even in high noise conditions, and with the lower observed source level, the whales could likely hear the snapping shrimp from Boat Rock well past the visual survey range of $2 \mathrm{~km}$. This range is likely an overestimate due to a large variety of parameters, including the given source level, transmission loss, noise level and selection of the detection threshold. Detection thresholds for marine mammals are not well categorized, and could vary greatly among individuals depending on their hearing abilities. However, even if the detection threshold for a whale was $10 \mathrm{~dB}$ or greater, these SNRs are still achieved at $2 \mathrm{~km}$. This means that snapping shrimp sounds are likely to always be audible at $2 \mathrm{~km}$ to the approaching whales, during all of the survey conditions.

The modeling here of the sound field presented to the whales as they approach Boat Rock is basic and subject to sources of error and uncertainty. The estimated levels of the snapping shrimp from the SHRU match very well with those of a shrimp measured in the lab. There are very little data on the source levels of snapping shrimp from actual rocky reefs in the ocean, all studies thus far document received levels of the shrimp as 
hydrophones pass over the beds. So this represents one of the first calculations for the source levels of snapping shrimp in situ. However, the snapping shrimp on Boat Rock were modeled as a point source, which may underestimate the actual levels produced by many snapping shrimp present on most faces of the rock. A study by Radford et al. (2011) showed that the actual detection range of biological sounds from a reef was much greater than the distance estimated by either spot measurements or spreading from a point source. This suggests that the detection range of Boat Rock could be even greater than estimated here.

Additionally, the noise levels used here were estimated according to Wenz (1962), and are actually about 5 to $10 \mathrm{~dB}$ higher than those estimated for Australian waters (Cato 1976). These decreased noise levels are likely due to the reduced amounts of shipping in Australian waters, which often dominates at lower frequencies in the northern hemisphere. So, it is possible that the actual noise levels present in this study are lower than what is estimated here. This would potentially increase the SNR seen at 2 $\mathrm{km}$.

The calculations of SNR are also highly dependent upon the estimations for TL, $\mathrm{BL}$, and surface loss. The values used here for each of these losses are a best estimation based upon the known environment. The loss factors calculated from the handheld hydrophone indicate that the estimation of TL as $10 \log \left(\frac{r}{r_{r e f}}\right)$ is appropriate for this location. However, variations in these loss values could have large effects on the minimum and maximum distances that the snapping shrimp signal could be detected. It is possible that the detection range for Boat Rock could be much reduced from those estimated if any of these factors are significantly different than what is modeled.

However, according to the given model, it seems likely that the detection range of Boat Rock is well beyond the $2 \mathrm{~km}$ visual range of this study. While it is possible that the detection range of Boat Rock goes under $2 \mathrm{~km}$ during sea states higher than those studied here, visual sighting of humpback whales becomes severely limited during these conditions, and it would therefore be extremely difficult to quantify any behavioral 
reaction. The fact that the sounds of snapping shrimp at Boat Rock are detectable to whales beyond the maximum range at which they could be visually tracked may explain why the visual analysis did not detect differences in the tracks during the different noise conditions.

In addition, the ability of the whales to detect and locate the obstacle at ranges of many kilometers brings into question the assumption that the whales react to an obstacle as soon as they detect it. If the whales can hear the signals from snapping shrimp at ranges well beyond those that pose a risk of collision, even in high noise conditions, then there would be no reason for them to initiate an avoidance reaction as soon as they detect Boat Rock. They would always be aware of its presence, during all the periods of visual observations, and therefore not need to change their behavior based on detection range. If they are in fact always aware of the island's presence and location, they would not need to initiate an avoidance reaction until they were close enough that collision with the island became a concern, a range on the order of tens of meters. This means that even if the detection range is in fact under $2 \mathrm{~km}$ during certain conditions, the whales are still likely to be able to detect it well before it becomes hazardous.

The fact that the sounds of the snapping shrimp from Boat Rock were found to always be detectible out to the maximum range of the visual observations lends further credence to the possibility that the whales are using snapping shrimp as an acoustic beacon to detect obstacles. Snapping shrimp are extremely well-suited as an acoustic indicator of an obstacle's locations because they form a loud, continuous signal that can be heard from great distances. Snapping shrimp, and other biologically produced reef sounds, have been shown to guide larval fish and invertebrates to reef settling sites (Jeffs et al. 2003, Simpson et al. 2005, Heenan et al. 2008), even potentially providing information on the suitability of the site for settling (Radford et al. 2010). If this type of information can be obtained by larval fish, whose hearing capabilities are not entirely known, then humpback whales, which are attuned to the acoustic environment, should be able to use the same information to detect the presence of these rocky reefs and islands. 
The potential use of snapping shrimp sounds has even been recognized by humans in the idea of 'acoustic daylight'. This is a concept useful for many naval applications, in which snapping shrimp sounds have been used to ensonify objects and passively create pictorial images of objects in the ocean (Epifanio et al. 1999). This type of passive echolocation could potentially even be possible for migrating whales, who are likely to use all acoustic information available to them.

\subsection{Conclusions}

The initial design of this study focused on discerning any differences in the behavior of migrating humpback whales that would indicate their perception, and use, of acoustic cues to detect near-shore obstacles. It was predicted that the whales would initiate an avoidance reaction when they were able to detect the island's presence, and that the range of this reaction would give an indication of whether or not they were using acoustic cues as a means of detecting the obstacle. However, when the visual tracks of humpback whales approaching a rocky island were examined, no consistent patterns were found that indicated a discernible avoidance of the island. Additionally, there were no differences in the movement patterns of the whales around the island during different proposed acoustic conditions, when either the sounds of crashing waves or snapping shrimp were proposed as the sound signal. However, modeling of the acoustic propagation of the snapping shrimp signals from the island revealed that these sounds are likely easily discernible at ranges well past the visual limit of this survey. The farranging nature of these sounds, even in high noise conditions, may explain why there were no differences in the behavior of the whales as they approached Boat Rock during different weather conditions. In this case, the lack of a behavioral reaction to the rock then became a confirmation that the whales are likely sensing it at all times, and therefore no behavioral reaction was necessary.

The fact that the sounds from the snapping shrimp could be heard kilometers away from their source lends credence to the idea that these types of sounds could serve as a long-distance acoustic indicator of an obstacle. It has long been theorized that sound 
is the most suitable distance sense available in the ocean. Humpback whales are already attuned to low frequency sound generation and reception, and therefore have the ability to utilize all the available sources of acoustic information in the ocean. Snapping shrimp present just one possible acoustic cue available to them. While snapping shrimp are the most prevalent biological generators of intense sound in the ocean, there are a large number of other species that produce noise as well. Some species may have smaller ecological ranges than snapping shrimp, and therefore, the combination of biological sounds produced may be specific to the species makeup of an area. This could allow the biotic acoustic signals to serve as a soundscape indicator of a relatively precise habitat or bathymetry type.

While the sounds of crashing waves were not addressed in depth here, due to the limits of literature values, and possibly due to interfering noise at low frequencies on the hydrophones, it is still possible that abiotic noise can serve as an orientation cue. The potential lower frequencies of these sounds mean that they could have an even greater range than that of biologically produced sound. While only short scale migration movements are addressed here, humpback whales also cover vast ocean distances, locating their destinations with astounding precision. It is possible that the combinations of biotic and abiotic sounds could serve as navigational beacons that can be heard by a whale while it is still many kilometers offshore, and could then be utilized to home in on its destination. So while acoustic cues could be useful on the short scales studied here, the combination of the species makeup of a location, along with abiotically produced sounds could also serve as a far ranging sound beacon for a whale navigating to a relatively specific location.

\subsection{Future work}

While this study has established that snapping shrimp could be useful as a long distance indicator of a location, there is still a considerable amount of work that could be done on this topic. The acoustic propagation calculations performed here are basic and contain many sources of potential error. Future studies should focus on more fully 
quantifying the sound field in a given area. This could be accomplished by deploying receivers at varying distances from the target island. This would allow for a more accurate quantification of the loss conditions for the site and the determination of the actual levels present at successively greater distances. In addition, during times of strong currents, strum noise on the hydrophones masked any lower frequency sounds that might have been present from the island. This problem could be addressed by a bottom mounted hydrophone for extended deployments, or a shorter term drifter with a hydrophone on it. In addition, the acoustic modeling would be greatly improved with detailed mapping of the bathymetry of the area, including information on the bottom type along the entire approach to the island.

The addition of several sensors to the hydrophone moorings would aid in quantifying the sound field as well. Salinity sensors attached alongside the temperature sensors would allow for more accurate calculation of the sound speed over the duration of the deployment. Furthermore, the weather measurements that were used here as an estimate for the background noise levels, were taken by observers on a cliff top, which could introduce some error. Equipping the hydrophone moorings with sensors to measure swell period and height as well as wind speed at the surface of the water, would allow for much more accurate assessment of the weather conditions that are influencing the background noise levels.

Additionally, playback studies would be useful in assessing whether or not migrating whales are in fact using sound cues for orientation. Acoustically simulating an island in an otherwise unobstructed area, where there are no other cues that could indicate its presence, and then monitoring the reactions of passing whales would give an indication of whether or not they are using sounds as an orientation cue. In 2011, I attempted to conduct this type of study in the same location as that of the 2010 study. A J11 transducer was deployed from a boat anchored $1 \mathrm{~km}$ to the south of Boat Rock. Northern migrating whales were tracked from the same location as 2010 , as one of three playback scenarios was conducted. The first was playback of sounds recorded by the SHRU off of Boat Rock during 2010. This was meant to acoustically simulate the island 
in an area where the island was not actually present. A white noise playback was also conducted on migrating whales in order to control for any effects of the playback itself. A silent control was also conducted to account for any effects of the boat. Unfortunately, extended bad weather did not allow for a large sample size, and therefore the playback study was inconclusive. However, this does not negate the importance of such a study being conducted in the future.

\subsection{Acknowledgements}

This project would not have been possible without the contributions of numerous people. The visual data collection was organized by Dr. Mike Noad and Dr. Rebecca Dunlop, including all volunteer recruitment and training. The visual observations would not have been possible without the help of numerous dedicated volunteers. Additional thanks go to Dave Paton who aided in the deployment and retrieval of the SHRU. Keith von der Heydt provided invaluable help in the setup of the SHRU for the project and in training me in its operation.

The analysis of the data was also only possible due to contributions from many different people. Art Newhall was invaluable in guiding analysis of the acoustic records and in providing the program to obtain the received levels from the SHRU. Dr. David Knobles kindly provided the bottom loss estimations for the propagation modeling. Dr. Jim Lynch was the main source of essential comments, guidance and direction in forming the sound propagation calculations. Dr. Peter Tyack provided significant insight, guidance and essential critiques of the methods along the way.

\subsection{References}

Addlington, R. 1963. Acoustic reflection losses at the sea surface measured with explosive sources. The Journal of the Acoustical Society of America 35:1834-1835.

Au, W. W. and K. Banks. 1997. The acoustics of snapping shrimps Synalpheus parneomeris in Kaneohe Bay. The Journal of the Acoustical Society of America 101:3032-3032. 
Au, W. W. L., A. Frankel, D. A. Helweg and D. H. Cato. 2001. Against the humpback whale sonar hypothesis. IEEE Journal of Oceanic Engineering 26:295-300.

Beaman, R. 2010. Project 3DGBR: a high-resolution depth model for the Great Barrier Reef and Coral Sea. Marine and Tropical Sciences Research Facility (MTSRF) Project $2: 13$.

Brekhovskikh, L. M. and I. U. r. P. Lysanov. 2003. Fundamentals of ocean acoustics. Springer Verlag.

Cato, D. 1976. Ambient sea noise in waters near Australia. The Journal of the Acoustical Society of America 60:320.

Cato, D. and R. D. McCauley. 2002. Australian research in ambient sea noise. Acoustics Australia 30:13-20.

Cato, D. H. and M. J. Bell. 1992. Ultrasonic ambient noise in Australian shallow waters at frequencies up to $200 \mathrm{kHz}$. DTIC Document. $27 \mathrm{pp}$.

Cato, D. H. and S. Tavener. 1997. Ambient sea noise dependence on local, regional and geostrophic wind speeds: implications for forecasting noise. Applied Acoustics 51:317338 .

Deane, G. B. 1997. Sound generation and air entrainment by breaking waves in the surf zone. The Journal of the Acoustical Society of America 102:2671.

Deane, G. B. 2000. Long time-base observations of surf noise. The Journal of the Acoustical Society of America 107:758-770.

Epifanio, C. L., J. R. Potter, G. B. Deane, M. L. Readhead and M. J. Buckingham. 1999. Imaging in the ocean with ambient noise: the ORB experiments. The Journal of the Acoustical Society of America 106:3211.

Everest, F. A., R. W. Young and M. W. Johnson. 1948. Acoustical characteristics of noise produced by snapping shrimp. The Journal of the Acoustical Society of America 20:137-142.

Heenan, A., S. Simpson and V. Braithwaite. 2008. Testing the generality of acoustic cue use at settlement in larval coral reef fish. Pages 7-11 Proc. of the 11 th Int. Coral Reef Symp., Fort Lauderdale, Florida.

Helweg, D., D. Houser and P. Moore. 2000. An integrated approach to the creation of a humpback whale hearing model. DTIC Document. $20 \mathrm{pp}$. 
Jeffs, A., N. Tolimieri and J. C. Montgomery. 2003. Crabs on cue for the coast: the use of underwater sound for orientation by pelagic crab stages. Marine and Freshwater Research 54:841-845.

Johnson, M. W., F. A. Everest and R. W. Young. 1947. The role of snapping shrimp (Crangon and Synalpheus) in the production of underwater noise in the sea. Biological Bulletin 93:122-138.

Kastelein, R. A., D. de Haan and W. C. Verboom. 2007. The influence of signal parameters on the sound source localization ability of a harbor porpoise (Phocoena phocoena). The Journal of the Acoustical Society of America 122:1238-1248.

Knudsen, V. O., R. Alford and J. Emling. 1948. Underwater ambient noise. The Journal of Marine Research 7:410-429.

Ma, B. B., J. A. Nystuen and R.-C. Lien. 2005. Prediction of underwater sound levels from rain and wind. The Journal of the Acoustical Society of America 117:3555.

Madsen, P. 2005. Marine mammals and noise: Problems with root mean square sound pressure levels for transients. The Journal of the Acoustical Society of America $117: 3952$.

Malme, C. I., P. R. Miles, C. W. Clark, P. L. Tyack and J. E. Bird. 1984. Investigations of the potential effects of underwater noise from petroleum industry activities on migrating gray whale behavior. U.S. Department of the Interior, Alaska OCS Office

Montgomery, J. C., A. Jeffs, S. D. Simpson, M. Meekan and C. Tindle. 2006. Sound as an orientation cue for the pelagic larvae of reef fishes and decapod crustaceans. Advances in Marine Biology 51:143-196.

Newhall, A. E., T. F. Duda, K. Von der Heydt, J. D. Irish, J. N. Kemp, S. A. Lerner, S. P. Liberatore, Y.-T. Lin, J. F. Lynch and A. R. Maffei. 2007. Acoustic and oceanographic observations and configuration information for the WHOI moorings from the SW06 experiment. DTIC Document. 116 pp.

Noad, M. J., D. H. Cato and M. D. Stokes. 2004. Acoustic tracking of humpback whales: measuring interactions with the acoustic environment. Pages 353-358. Proceedings of Acoustics Gold Coast, Australia.

Noad, M. J., D. Paton and D. H. Cato. 2006. Absolute and relative abundance estimates of Australian east coast humpback whales (Megaptera novaeangliae). Paper $\mathrm{SC} / 59 / \mathrm{SH} 31$ presented to the IWC Scientific Committee. Hobart, Tasmania 
Norris, K. S. 1967. Some observations on the migration and orientation of marine mammals. Pages 101-125 R. M. Storm ed.in Animal Orientation and Navigation. Corvallis: Oregon State University Press.

Payne, R. S. and S. McVay. 1971. Songs of Humpback Whales. Science 173:585-597.

Plomp, R. and M. Bouman. 1959. Relation between hearing threshold and duration for tone pulses. The Journal of the Acoustical Society of America 31:749.

Radford, C., J. Stanley, C. Tindle, J. Montgomery and A. Jeffs. 2010. Localised coastal habitats have distinct underwater sound signatures. Marine Ecology Progress Series 401:21-29.

Radford, C. A., A. G. Jeffs, C. T. Tindle and J. C. Montgomery. 2008. Temporal patterns in ambient noise of biological origin from a shallow water temperate reef. Oecologia 156:921-929.

Radford, C. A., C. T. Tindle, J. C. Montgomery and A. G. Jeffs. 2011. Modelling a reef as an extended sound source increases the predicted range at which reef noise may be heard by fish larvae. Marine Ecology Progress Series 438:167-174.

Readhead, M. L. 1997. Snapping shrimp noise near Gladstone, Queensland. The Journal of the Acoustical Society of America 101:1718.

Richardson, W. J. and D. H. Thomson. 1998. Marine mammals and noise. Academic Press, San Diego, CA.

Simpson, S. D., M. Meekan, J. Montgomery, R. McCauley and A. Jeffs. 2005. Homeward sound. Science 308:221-221.

Smith, J., H. S. Grantham, N. Gales, M. C. Double, M. J. Noad and D. Paton. 2012. Identification of humpback whale breeding and calving habitat in the Great Barrier Reef. Marine Ecology Progress Series 447:259-272.

Tolimieri, N., A. Jeffs and J. C. Montgomery. 2000. Ambient sound as a cue for navigation by the pelagic larvae of reef fishes. Marine Ecology Progress Series 207:219224.

Urick, J. R. 1983. Principles of underwater sound. McGraw-Hill Book Company, New York, NY.

Urick, R. J. 1984. Ambient noise in the sea. DTIC Document. 193 pp. 
Versluis, M., B. Schmitz, A. von der Heydt and D. Lohse. 2000. How snapping shrimp snap: through cavitating bubbles. Science 289:2114-2117.

Wartzok, D. and D. R. Ketten. 1999. Marine Mammal Sensory Systems. J. E. Reynolds lii and S. A. Rommel eds.in Biology of Marine Mammals. Smithsonian Institution.

Wenz, G. M. 1962. Acoustic ambient noise in the ocean: spectra and sources. The Journal of the Acoustical Society of America 34:1936-1956.

Wilson Jr, O., S. N. Wolf and F. Ingenito. 1985. Measurements of acoustic ambient noise in shallow water due to breaking surf. The Journal of the Acoustical Society of America 78:190-195.

Zhou, J.-X., X.-Z. Zhang and D. Knobles. 2009. Low-frequency geoacoustic model for the effective properties of sandy seabottoms. The Journal of the Acoustical Society of America 125:2847. 


\section{Chapter 4}

\section{Analysis of a Blainville's beaked whale's movement response to playback of killer whale vocalizations}

This chapter was originally published online in Marine Mammal Science and is reproduced here with their permission

Ann N. Allen, Julian J. Schanze, Andrew R. Solow and Peter L. Tyack. 2013. Analysis of a Blainville's beaked whale's movement response to playback of killer whale vocalizations. Article first published online: 1 Apr 2013. doi: 10.1111/mms.12028 


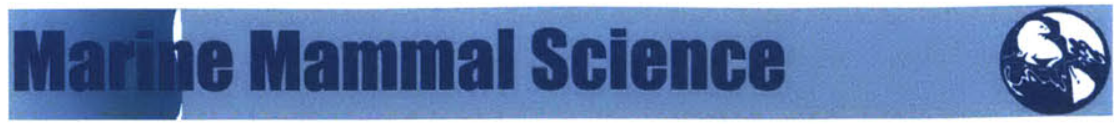

MARINE MAMMAL SCIENCE, $* *(*): * * * * * *(* * * 2013)$

(C) 2013 by the Society for Marine Mammalogy

DOI: $10.1111 / \mathrm{mms} .12028$

\title{
Analysis of a Blainville's beaked whale's movement response to playback of killer whale vocalizations
}

Ann N. Allen, ${ }^{1}$ Julian J. Schanze and Andrew R. Solow, Woods Hole Oceano graphic Institution, 266 Woods Hole Road, Woods Hole, Massachusetts 02543, U.S.A.; Peter L. TyACK, Sea Mammal Research Unit, Scottish Oceans Institute, East Sands, Univer sity of St Andrews, Fife, KY16 8LB, United Kingdom.

\begin{abstract}
Increasing evidence links exposure to Navy sonar with certain mass stranding events of deep diving beaked whales. Although the cause of these strandings is unknown, one theory suggests that the animals confuse the sonar signals with vocal izations of killer whales, a known predator. Here we analyze the movement patterns of a tagged female Blainville's beaked whale in reaction to playback of killer whale predation calls. During a deep foraging dive, the whale was exposed to a playback of killer whale vocalizations with the source level slowly increased until the whale pre maturely ceased foraging. The heading data from the tag were analyzed using a rota tion test with a likelihood ratio calculated for a nonparametric kernel density estimate. We found a significant difference $(P<0.005)$ in the distribution of $\Delta$ heading (the change in heading averaged over $200 \mathrm{~s}$ ) after the cessation of the killer whale playback. A test of the angular standard deviation (SD) of the $\Delta$ heading showed that after the playback, the SD was significantly reduced $(P=0.0064)$, which indicates that the animal maintained a straighter than normal course for an extended period of time. The prolonged directed avoidance response observed here suggests a behavioral reaction that could pose a risk factor for stranding.
\end{abstract}

Key words: beaked whale, Navy sonar, mass stranding, behavioral response, killer whale predation response, mid frequency active sonar, Mesoplodon densirostris.

Increasing anthropogenic noise in the ocean and its effects on marine life has become a rising concern for lawmakers and researchers alike in recent years. Of partic ular concern are those marine animals that utilize sound to communicate and explore their environment, such as marine mammals. Human contributions to noise in the ocean, including shipping, oil and gas development, and military activities, have greatly increased in the last $50 \mathrm{yr}$ (McDonald et al. 2008). While most of the concern centers around the effects of low frequency sound on baleen whales, which can range from changes in the vocal behavior of the whales (Parks et al. 2007) to abandonment of habitat (Bryant et al. 1984), the most immediate and extreme consequences of anthropogenic sounds are the mass strandings of beaked whales associated with mili tary mid frequency active (MFA) sonar exercises.

\footnotetext{
${ }^{1}$ Corresponding author (e-mail: anallen@whoi.edu).
} 
Starting in the late 1990 s, evidence began to accumulate that atypical mass stran dings of several species of beaked whales were associated with military sonar activities (Frantzis 1998). There have been 12 mass stranding events associated with the pre sence of naval exercises or warships outfitted with MFA sonar, ranging in location from the Bahamas to the Mediterranean (D'Amico et al. 2009). These sonar related mass strandings have mainly involved Cuvier's (Ziphius cavirostris) and Blainville's (Mesoplodon densirostris) beaked whales.

Beaked whales are extreme deep divers, with Blainville's beaked whales regularly conducting foraging dives to depths in excess of $1,000 \mathrm{~m}$ (Tyack et al. 2006). At depth they emit echolocation clicks with frequencies centered around $40 \mathrm{kHz}$ and with little energy below $20 \mathrm{kHz}$ (Zimmer et al. 2005). Acoustic tags have recorded echoes of these clicks from prey items, providing direct evidence that these clicks are used in foraging (Johnson et al. 2004). One study has shown that Blainville's beaked whales produce these echolocation clicks at depth for an average of $26 \mathrm{~min}$ and have an average total dive duration of $47 \mathrm{~min}$ (Tyack et al. 2006). The deep diving and infrequent surfacing behavior of beaked whales make them very difficult to study, yet they exhibit one of the most dramatic and lethal responses of marine mammals to human activities. Determining what factors cause beaked whales to mass strand is an important step in guiding regulation of sonar use in order to minimize its effects on beaked whales.

There has been extensive speculation as to what leads to the stranding and death of beaked whales during navy MFA sonar exercises. Initially it was hypothesized that the sonar caused direct physical damage to the whales, due to the presence of gas bub ble lesions and subarachnoid hemorrhages observed in stranded animals (Evans and England 2001, Jepson et al. 2003) and the potential for intense sound energy to cause bubbles to grow in supersaturated tissues (Crum and Mao 1996). More recent hypotheses have focused on the possibility that sonar initiates a chain of events that lead to strandings but starts with a purely behavioral reaction. Beaked whales live in deep waters, so they must show a strong avoidance reaction to swim from their normal habitat onto the beach (Cox et al. 2006).

The frequency of MFA sonar ranges from 2.6 to $14 \mathrm{kHz}$ (D'Amico et al. 2009), which is well below the best hearing range of beaked whales (Cook et al. 2006, Finn eran et al. 2009). However, the sonar signals are acoustically similar to the stereotyped calls of killer whales (Orcinus orca), a primary predator of beaked whales (Zimmer and Tyack 2007). It has been hypothesized that the MFA sonar signal may initiate a pred ator avoidance reaction in the beaked whales, similar to the reaction elicited by killer whales, that may lead to stranding (Zimmer and Tyack 2007). Studies of killer whale predation on large baleen whales have shown that baleen whales employ two basic strategies for avoiding killer whale predation: fight and flight (Ford and Reeves 2008). Those species that employ a flight strategy attempt to outdistance the killer whales by maintaining a straight heading at high speeds over an extended time period (Ford et al. 2005, Ford and Reeves 2008). Of the flight species, both minke (Balaenop tera acutorostrata) and sei whales (Balaenoptera borealis) have been observed to strand themselves in attempts to escape predation by killer whales (Ford et al. 2005, Ford and Reeves 2008). In most cases the stranding itself leads to eventual death, but in rare cases the fleeing whale succeeded in swimming away when the tide rose and thus effectively escaped killer whale predation (Ford and Reeves 2008). It has been hypoth esized that beaked whales may employ an avoidance strategy similar to these whales, and that the strandings are the result of either mistaken direction during flight, or a deliberate action taken to avoid what they may perceive as an immediate threat. 


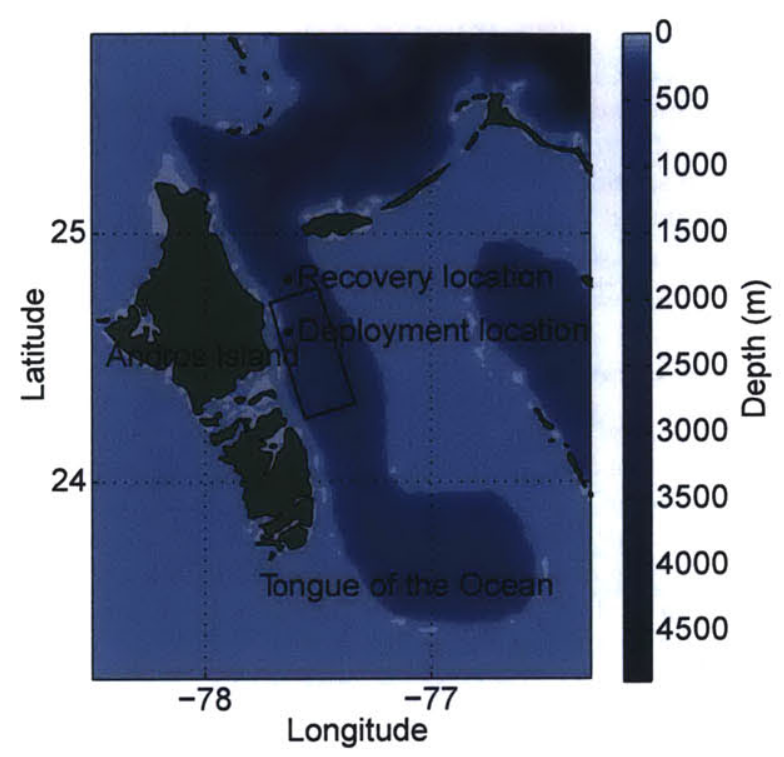

Figure 1. A map of the location of the study area in the Tongue of the Ocean, near Andros Island, Bahamas. The black box indicates the area containing the AUTEC hydrophone array, and the deployment and recovery locations of the Dtag are marked. The two locations are approximately $24 \mathrm{~km}$ apart. Bathymetry data from Amante and Eakins (2009).

Understanding what factors lead beaked whales to strand during navy sonar exer cises is an important step in determining how to reduce the risk of these activities. However, the elusive nature of these animals and the diverse factors involved in each stranding incident lead to extreme difficulty in studying this problem.

This paper utilizes a controlled exposure experiment to test one beaked whale's reaction to MFA sonar signals and the calls of mammal eating killer whales filtered to a frequency bandwidth similar to that of MFA sonar. This experiment was designed to test the above hypothesis that beaked whales respond to killer whale pre dation calls with a directed prolonged avoidance reaction similar to the flight response of baleen whales. We use the heading data from a tagged beaked whale to develop a method of statistical analysis of avoidance reactions and discuss the implica tions of the observed reaction.

\section{Methods}

\section{Field Site}

To reduce some of the difficulty associated with locating beaked whales for study, the experiment was conducted on the Atlantic Undersea Test and Evaluation Center (AUTEC) near Andros Island, Bahamas. AUTEC is located in the Tongue of the Ocean (TOTO), a deep water canyon (maximum depth $\sim 2,000 \mathrm{~m}$ ) that runs roughly north south with the only deep water entrance located at the northern end (Fig. 1). 
This area is home to three species of beaked whales, with Blainville's beaked whale being the most common (Claridge 2006). Roughly 25 Blainville's beaked whales use TOTO as a foraging ground at any one time (Marques et al. 2009). This canyon was chosen as a study site due to the presence of an array of 82 hydrophones installed by the U.S. Navy on the sea floor of the AUTEC range. A marine mammal monitoring program has been installed to localize the echolocation clicks of Blainville's beaked whales in real time (Ward $e$ al. 2008) and this system was utilized during the study to monitor the clicking of the tagged whale.

\section{Digital Acoustic Recording Tagging}

This study used a digital acoustic recording tag (Dtag), which is an archival suc tion cup tag that contains a pressure sensor and three axis magnetometers and acceler ometers that measure depth, pitch, roll, and heading of the tagged whale at a sample rate of $50 \mathrm{~Hz}$ (Johnson and Tyack 2003). In addition, two hydrophones record acous tic data at a sampling rate of $192 \mathrm{kHz}$ (Johnson and Tyack 2003). The tag is designed for deployments of up to $17 \mathrm{~h}$, and is attached to the whale via four suction cups. The tag releases at a preprogrammed time, and is tracked and recovered utiliz ing a VHF radio transmitter.

\section{Tagged Whale}

A Dtag was deployed on a female Blainville's beaked whale on 2 September 2007 within the AUTEC range. For the duration of the deployment, the whale was tracked while at the surface utilizing the VHF radio beacon on the tag. The whale was moni tored during its first three foraging dives by localizing its echolocation clicks via the AUTEC hydrophone array. When possible, visual sightings of the tagged whale at the surface were utilized to augment the tracking data.

\section{Playbacks}

The tagged whale was exposed to two stimuli: an MFA sonar signal and vocaliza tions of marine mammal-eating killer whales. All playbacks were conducted utiliz ing a Naval Undersea Warfare Center (NUWC) Eryn I MFA source. The source is capable of transmitting MFA sonar signals and other broadband sounds in the 2$5 \mathrm{kHz}$ band, up to a source level (SL) of $212-214 \mathrm{~dB}$ re $1 \mu \mathrm{Pa}$ at $1 \mathrm{~m}$. The beam pattern is somewhat directional, with more of the output acoustic energy directed near to the horizontal plane of the source. For the duration of the playbacks, the transducer was deployed from the M/V Ranger at a depth of $45 \mathrm{~m}$ while the ship drifted at a distance of approximately $1 \mathrm{~km}$ from where the tagged whale began its deep foraging dives.

After the whale conducted a single preexposure dive and began a second foraging dive, an MFA sonar playback was performed. Playback was not initiated until forag ing began, indicated by reception of echolocation clicks on the AUTEC array. The MFA sonar signal was designed to simulate an actual waveform transmitted by the U.S. Navy. It was composed of three sequential components: a $0.5 \mathrm{~s}$ linear frequency modulated upsweep from 3.2 to $3.3 \mathrm{kHz}$, a $0.5 \mathrm{~s}$ constant frequency tone of $3.43 \mathrm{kHz}$, a $0.1 \mathrm{~s}$ silent interval and a $0.3 \mathrm{~s}$ constant frequency tone of $3.75 \mathrm{kHz}$. The signal sequence was repeated every $25 \mathrm{~s}$. The playback started at a source level (SL) of $152 \mathrm{~dB}$ re $1 \mu \mathrm{Pa}$ at $1 \mathrm{~m}$, and was increased by $3 \mathrm{~dB}$ every $25 \mathrm{~s}$. The playback 
protocol called for continual increase of the SL until echolocation clicks from the for aging whale were no longer heard on the AUTEC hydrophone array, or a maximum SL of $212 \mathrm{~dB}$ re $1 \mu \mathrm{Pa}$ at $1 \mathrm{~m}$ was achieved.

Once the tagged whale started producing echolocation clicks on the third posttag ging dive, playback of the killer whale predation calls was initiated. The transmitted killer whale sounds consisted of a 10 min segment of recordings from wild marine mammal eating killer whales recorded in southeast Alaska. The killer whale calls were band pass filtered to a range of $2-5 \mathrm{kHz}$, in order to match the frequency range of the transducer (Fig. S1). The killer whale playback was initiated at a SL of 130-140 dB re $1 \mu \mathrm{Pa}$ at $1 \mathrm{~m}$, and then increased by $5 \mathrm{~dB}$ every $30 \mathrm{~s}$, reaching a maximum of 190 $203 \mathrm{~dB}$ re $1 \mu \mathrm{Pa}$ at $1 \mathrm{~m}$. Playback was terminated several minutes after echolocation clicks ceased to be detected on the AUTEC array. Data from the whale were recorded continuously until the Dtag detached approximately $10 \mathrm{~h}$ later.

\section{Statistical Analysis}

The heading data recorded on the Dtag were used to conduct a statistical analysis to test if the tagged whale's movement patterns from before either the MFA sonar or the killer whale playback were different from those after each playback. The observed headings were averaged over nonoverlapping $200 \mathrm{~s}$ intervals in order to filter out any small scale variation in movements due to fuking motion, head scanning, etc. For this analysis, the change between subsequent averaged headings ( $\Delta$ heading), rather than the true heading of the whale, was utilized in order to test for patterns of change in movement. $\Delta$ Heading was calculated using $\mathrm{CircStat}$, a circular statistics toolbox for MATLAB (Berens 2009).

Let $\Delta_{1}, \Delta_{2}, \ldots, \Delta_{\tau}, \Delta_{\tau+1}, \ldots, \Delta_{n}$ be the time series of heading changes where $\tau$ is the time of the cessation of the playback, which approximates initiation of the whale's response to each playback. We assume that $\Delta_{1}, \Delta_{2}, \ldots, \Delta_{\tau}$ are independent and iden tically distributed with unknown probability density function $f_{B}(\Delta)$ and $\Delta_{1}, \Delta_{2}, \ldots, \Delta_{\tau}$ are also independent and identically distributed with probability density function $f_{A}(\Delta)$. We tested the null hypothesis: $H_{0}: f_{B}=f_{A}=f$ that heading changes before and after the playback have a common distribution against the alternative hypothesis: $H_{1}$ : $f_{B} \neq f_{A}$ that they do not.

The $\Delta$ heading data were used to conduct a nonparametric likelihood ratio (NLR) test to determine if the distributions of the data before and after the each playback were different. Under this model, the log likelihood is given by:

$$
\log L=\sum_{t=1}^{\tau} \log f_{B}\left(\Delta_{t}\right)+\sum_{t=\tau+1}^{n} \log f_{A}\left(\Delta_{t}\right)
$$

and the likelihood ratio statistic for testing $H_{0}$ against $H_{1}$ is:

$$
\text { NLR }=\left(\log L_{1}-\log L_{0}\right)
$$

where $\log L_{1}$ and $\log L_{0}$ are the maximized values of the $\log$ likelihood under $H_{0}$ and $H_{1}$, respectively. In the absence of suitable parametric models for $f_{B}$ and $f_{A}$, a NLR can be formed using: 


$$
\log L=\sum_{t=1}^{\tau} \log \hat{f}_{B}\left(\Delta_{t}\right)+\sum_{t=\tau+1}^{n} \log \hat{f}_{A}\left(\Delta_{t}\right)
$$

and

$$
\log L_{0}=\sum_{t=1}^{n} \log \hat{f}\left(\Delta_{t}\right)
$$

where $\hat{f}_{B}$ is a nonparametric kernel estimate of $f_{B}$ based only on $\Delta_{1}, \Delta_{2}, \ldots, \Delta_{\tau}, \hat{f}_{A}$ is a nonparametric kernel estimate of $f_{A}$ based only on $\Delta_{\tau+1}, \Delta_{\tau+2}, \ldots, \Delta_{\mathrm{n}}$ and $f$ is a nonparametric kernel estimate of $f$ based on the entire time series. This is an example of a nonparametric likelihood ratio statistic (Cao and Van Keilegom 2006).

To assess the significance of the observed value of the NLR statistic, we used the rotation method of DeRuiter and Solow (2008). This involved transforming the time series into a circle by joining its end to its beginning and then rotating the order by one sample. The NLR statistic was then calculated for the rotated time series, using the same breakpoint position as the observed data. This process was repeated for each rotation position until we stepped through the entire time series. The observed signifi cance level (or $P$ value) was then estimated by the proportion of rotated time series for which NLR exceeded the observed value. The advantage of this approach is that, except for a negligible end effect, it preserves any serial dependence in the rotated time series of $\Delta$ heading. Such serial dependence can undermine the validity of a standard randomization test in which the time series is randomly scrambled (Manly 2006).

The kernel density estimate (KDE) for the angular data was calculated according to Fisher (1995). Briefly, the KDE based on observations $\Delta_{1}, \Delta_{2}, \ldots, \Delta_{m}$ has the form:

$$
\hat{f}(\Delta)=(m b)^{1} \sum_{j=1}^{m} K\left(\frac{\left|\Delta-\Delta_{j}\right|}{b}\right)
$$

where $K$ is a probability density function symmetric around $0,\left|\Delta \quad \Delta_{j}\right|$ is the angular difference between $\Delta$ and $\Delta$, and $b$ is the bandwidth that controls the smoothness of $\hat{f}$. Here we use the bisquare kernel:

$$
K(x)= \begin{cases}0.9375\left(1-x^{2}\right)^{2} & -1 \leq x \leq 1 \\ 0 & \text { otherwise }\end{cases}
$$

A common choice of bandwidth is:

$$
b_{S}=1.06 \hat{\sigma}_{\text {ang }} m^{1 / 5}
$$

where $\hat{\sigma}_{a n g}$ is the sample angular standard deviation of the observations. For cosmetic reasons, we used $b=1.5 b_{s}$. However, the results are insensitive to the choice of band width in this vicinity.

Standard Deviation Analysis

The nonparametric likelihood ratio test is designed to test for a general change in the distribution of $\Delta$ heading. To sharpen the analysis, we focused on detecting a 
change in the dispersion of $\Delta$ heading as measured by the angular standard deviation $\sigma_{a n g}$. Let $\hat{\sigma}_{a n g, B}$ and $\hat{\sigma}_{a n g, A}$ be the sample angular standard deviations formed from the data before and after the cessation of the killer whale playback, respectively. To test the null hypothesis $H_{0}: \sigma_{a n g, B}=\sigma_{a n, A}$ against the alternative hypothesis $H_{1}: \sigma_{a n g, B} \neq$ $\sigma_{a n g, A}$, we formed the absolute difference $\left|\hat{\sigma}_{a n g, A}-\hat{\sigma}_{a n g, B}\right|$. The significance of this absolute difference was assessed by the same rotation procedure outlined above. In this case, the $P$ value was approximated by the proportion of rotated time series for which the value of $\left|\hat{\sigma}_{a n g, A}-\hat{\sigma}_{a n g, B}\right|$ exceeded the observed value.

\section{RESULTS}

During August and September of 2007, we used a digital acoustic recording tag (Dtag) (Johnson and Tyack 2003) to conduct a behavioral response study of a Blain ville's beaked whale. The Dtag was deployed on an adult female Blainville's beaked whale at $24.6025^{\circ} \mathrm{N}, 77.6210^{\circ} \mathrm{W}$ on 2 September 2007 (Fig. 1).

After tag attachment, the whale conducted a deep dive that we considered a preex posure baseline dive. Clicks from the tagged whale were monitored on the AUTEC hydrophone array. After the whale initiated its second deep dive and was heard pro ducing echolocation clicks associated with foraging, the MFA playback was initiated. The whale ceased clicking $9 \mathrm{~min}$ after the start of playback, when the received level of the sonar signal at the tag was $138 \mathrm{~dB}$ re $1 \mu \mathrm{Pa}$ sound pressure level (SPL), with a cumulative sound exposure level of $142 \mathrm{~dB}$ re $1 \mu \mathrm{Pa}^{2}$ s (fig. 9, Tyack et al. 2011). The whale then ascended more slowly than usual and moved away from the sound source. The whale remained in the area for around $2 \mathrm{~h}$ and then commenced a third foraging dive (Tyack et al. 2011).

Once foraging clicks were initiated on the third dive, the whale was exposed to playback of the killer whale calls. The killer whale playback was also slowly ramped up as the tagged whale's clicking was monitored. The whale stopped clicking about $5 \mathrm{~min}$ into the playback, approximately $1 \mathrm{~min}$ after the received level of the killer whale sound reached $98 \mathrm{~dB}$ re $1 \mu \mathrm{Pa}$ SPL (fig. 10, Tyack et al. 2011). The whale then again made a slow ascent, the slowest analyzed from a set of 32 deep foraging dives from six whales tagged at this site (Tyack et al. 2011). After surfacing, the whale swam away from the playback location for approximately $10 \mathrm{~h}$, before the tag detached and was then recovered at $24.8136 \mathrm{~N}, 77.6265^{\circ} \mathrm{W}$, a location approxi mately $24 \mathrm{~km}$ away from the deployment site (Fig. 1).

Utilizing speed estimation from the pitch angle and the rate of change of depth recorded on the Dtag, a rough approximation of the tagged whale's path, called a pseudo track, was generated (Tyack et al. 2011) (Fig. 2). As seen in Fig. 2, after ces sation of the MFA sonar playback, the whale briefly maintained a course heading to the north. After several hours, the whale started a deep foraging dive. After cessation of the killer whale playback, the whale maintained a heading directed to the north for the remainder of the tag attachment (Fig. 3). If the whale continued on this course after tag detachment, it would have passed through the only deep water exit from the TOTO canyon.

In order to test whether the whale altered its movement patterns in response to either the MFA sonar or killer whale playback, we performed a rotation test of the heading data from the Dtag. We used a nonparametric likelihood ratio test (NLR) (Cao and Van Keilegom 2006) to determine if the distribution of $\Delta$ heading was dif ferent in the two periods: before and after cessation of the MFA and killer whale 


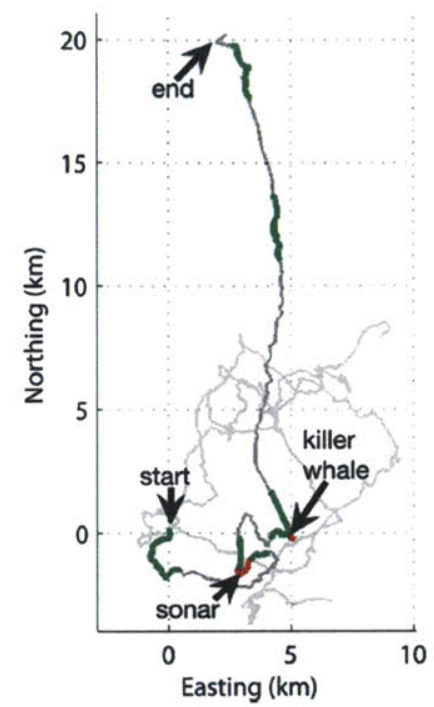

Figure 2. The estimated tracks of four tagged beaked whales. The horizontal track of the tagged whale is indicated in dark gray, with deep foraging dives marked in dark green. The sonar playback and killer whale playback are marked in red and labeled. The tracks of three other beaked whales tagged, but not exposed to playbacks, are indicated in light gray (repro duced from Tyack et al. 2011). A significant straightening of the whale's course is apparent after the end of the killer whale playback.

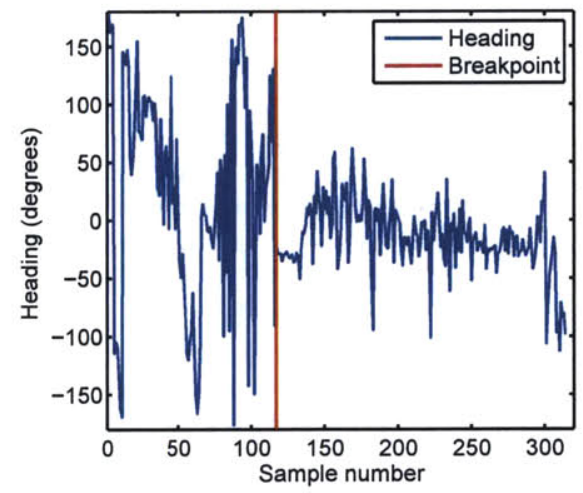

Figure 3. A time series plot of the heading data averaged over $200 \mathrm{~s}$ with the breakpoint in the data (cessation of the killer whale playback) marked by the red line. The variation in head ing is noticeably reduced after the breakpoint and mainly fluctuates around $0^{\circ} \mathrm{N}$.

playbacks. The kernel density estimate (KDE) was calculated for each of the time periods (Fig. 4) and we assessed the significance of the observed value of the NLR statistic via a discrete time version of a rotation test (Deruiter and Solow 2008). Of 312 NLR values generated using the breakpoint defined by cessation of the MFA playback, 146 

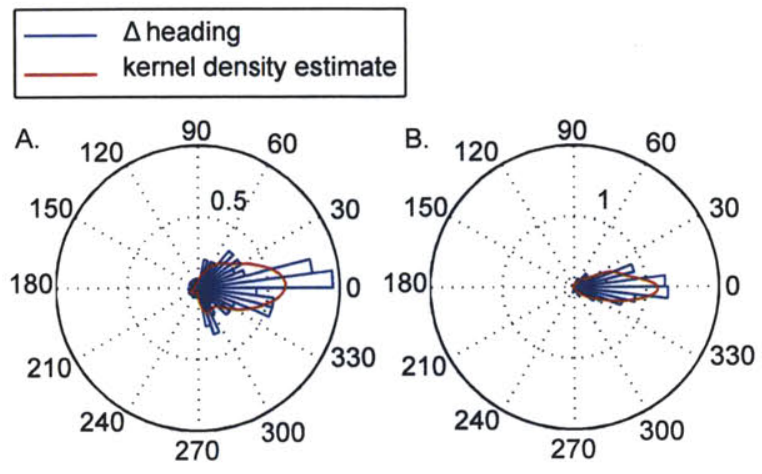

Figure 4. Angular histogram plots of $\Delta$ heading data in blue with the kernel density esti mate drawn in red. The distribution of $\Delta$ heading data before the killer whale breakpoint is illustrated in A, with the maximum occurrence of greater than 0.5 for the angles, and the dis tribution of $\Delta$ heading data after the killer whale breakpoint is shown in B, with a maximum occurrence of greater than 1 .

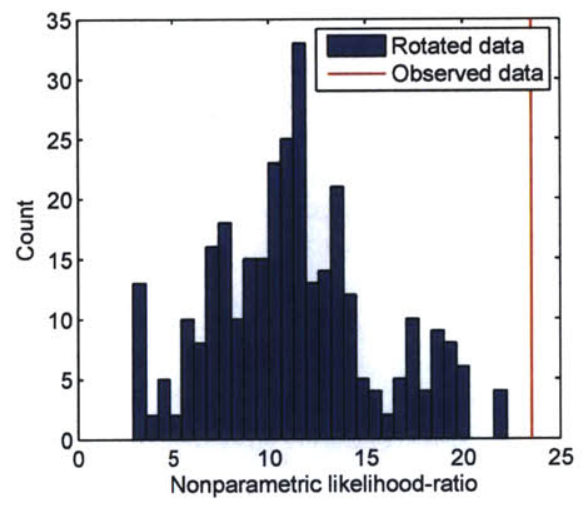

Figure 5. Comparison of rotated and observed nonparametric likelihood ratios (NLR) calculated for the killer whale playback breakpoint. The histogram shows the distribution of the NLRs calculated for rotated data order and the observed NLR is plotted in red. It is outside the range of all NLRs calculated for rotated data order $(P<0.005)$.

exceeded the observed value, giving a $P$ value of 0.468 (Fig. S2). This indicates that there is no significant change in the whale's movements after the cessation of the MFA sonar playback. Of 312 values of the NLR statistic generated in this way for the killer whale playback, none exceeded the observed value (Fig. 5) giving an esti mated $P$ value of $<0.005$. Therefore, we conclude that there is a significant difference in the whale's movement behavior between these two periods, as reflected in the distribution of $\Delta$ heading.

In order to further our understanding of how the beaked whale responded to the killer whale playback, we tested for a difference in the dispersion of $\Delta$ heading after the killer whale breakpoint, as measured by the angular standard deviation (Fisher 1995). As before, significance was assessed by rotating the order of the time series of 


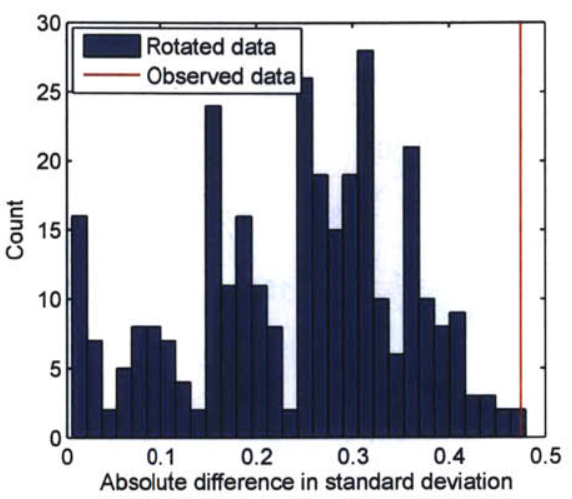

Figure 6. Comparison of the rotated and observed difference in absolute standard deviation. The histogram shows the distribution of the difference in angular standard deviation between before and after the killer whale breakpoint for all rotations of data order. The observed differ ence in standard deviation is shown in red and is greater than almost all of the values calculated via the rotation test $(P=0.0064)$.

$\Delta$ heading. Of the 312 values generated this way, two exceeded the observed value, giving an estimated $P=0.0064$ (Fig. 6). While the distributions of $\Delta$ heading both before and after the breakpoint are centered around zero, the angular standard devia tion of the data after the breakpoint was $27.4^{\circ}$ less than that before. This reduced standard deviation indicates that the tagged whale maintained a more directed course after the cessation of the killer whale playback.

\section{Discussion}

This study utilized a playback experiment to test the behavioral reaction of a tagged Blainville's beaked whale to MFA sonar and the calls of killer whales that feed on marine mammals. Due to the difficult nature of finding and tagging $M$. densirostris, this study represents the only playback experiment to date for these whales with an extended monitoring period after exposure. Determining what features of MFA sonar cause beaked whales to strand is an important but difficult task. A whale living in deep water must swim far from its typical habitat before it is at risk of stranding. Baleen whales avoiding predation by killer whales have been observed to strand (Ford et al. 2005, Ford and Reeves 2008), suggesting that directed avoidance in reaction to predators may increase a whale's risk of stranding. Therefore, we use heading data here to study whether a beaked whale responded to playback of MFA sonar or killer whale calls with a straighter course of travel that would cause it to swim far from its foraging site, potentially raising the risk of stranding. The small sample size limits the conclusions that can be drawn from the experimental scenario. However, utilizing the heading data from the Dtag, we are able to employ a novel statistical technique to draw some basic conclusions about the data.

During exposure to each of the playback stimuli the whale ceased clicking early in the deep foraging dive at a received level of $138 \mathrm{~dB}$ re $1 \mu \mathrm{Pa}$ SPL for the MFA play back and $98 \mathrm{~dB}$ re $1 \mu \mathrm{Pa}$ SPL for the killer whale playback. In each case, after cessa 

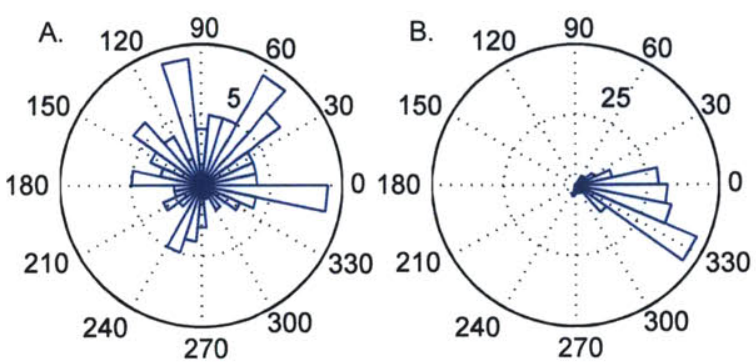

Figure 7. Angular histogram plots illustrating the distribution of heading data before the killer whale breakpoint $(A)$ and after the killer whale breakpoint (B). There is a maximum occurrence of greater than 5 for the heading angles in $\mathrm{A}$ and greater than 25 in $\mathrm{B}$. Clustering around the north $\left(0^{\circ}\right)$ to north west direction can be seen in $\mathrm{B}$.

tion of clicking, the whale initiated a slower than normal ascent to the surface (Tyack et al. 2011). While there is a temporary avoidance reaction to the MFA sonar playback, observed as a straightening of course (Fig. 2), the whale appeared to resume normal foraging about two hours after surfacing (Tyack et al. 2011). A test of the heading data before and after cessation of the MFA playback revealed that there were no sig nificant differences in the whale's heading after this playback (Fig. S2). An extended avoidance reaction was observed only after the killer whale playback. However, because the stimuli were played in sequence, we cannot rule out the possibility that the behavioral response was cumulative, and that the MFA sonar playback only several hours earlier had a potentiating effect on the response to the killer whale playback.

When the $\Delta$ heading data were split into two sections, a likelihood ratio test revealed that, when the cessation of the killer whale playback was used as the break point, the distributions for these two groups were significantly different (Fig. 5). Fur ther testing indicated a significant difference in the angular standard deviation of the $\Delta$ heading data, with the SD of the $\Delta$ heading distribution after the playback signifi cantly lower than would be predicted from rotated data (Fig. 6). This indicates that the whale maintained a more directed course after the cessation of the killer whale playback (Fig. 2, 3). The whale's course heading was centered on a northerly direc tion (Fig. 7), which took it directly away from the source of the playback, and towards the only deep water exit of the TOTO canyon.

It should be noted that, while the experiment was designed to test for a change in movement patterns, as measured by heading, the angular standard deviation test was developed post hoc. The NLR tests for any change in the distribution of the $\Delta$ heading data. Once it was determined that there was a significant difference between the distri bution of the whale's heading before and after the killer whale playback, we then chose to focus on the variation in heading, as measured by the angular standard deviation. This decision was influenced by the observed results, and ideally, the test developed after this examination of the data would be utilized to confirm these findings in future playback experiments. However, the difficulty involved in finding and tagging beaked whales made this unfeasible in this case. One goal for this paper is to encourage simi lar future playback experiments to use this method to test for similar responses.

This prolonged, directed avoidance in reaction to the killer whale playback put increasing distance between the whale and the location of the playback, similar to 
that seen in predation avoidance by other species. Minke and sei whales, which employ this flight strategy, have been observed to beach themselves while being chased by killer whales (Ford et al. 2005, Ford and Reeves 2008). The reaction observed here may be an antipredator response similar to the flight reaction of baleen whales to killer whale predators (Ford et al. 2005, Ford and Reeves 2008) and it is possible that this sustained directed flight puts beaked whales at risk for stranding as well. It is not apparent whether the strandings of baleen whales were the result of an intentional avoidance strategy, or if the whales inadvertently ran into the shallows due to their fixed course, or were perhaps driven ashore by the pursuing whales (Ford et al. 2005, Ford and Reeves 2008). Regardless of the reason for stranding, in only one observed case was a minke whale able to work its way off the beach after the killer whales departed. Therefore, if it is an intentional strategy, it must be a last ditch very high risk effort, motivated by extreme predation pressure. If Blainville's beaked whales utilize a similar strategy, then in extreme cases this may put them at risk for stranding. In this experiment, a prolonged avoidance response was observed for play back of killer whale sounds, but not for the short, low level playback of MFA sonar. However, if naval sonar exercises are either very loud, very extended or both, it is pos sible that they could elicit this same prolonged avoidance response in beaked whales that could lead to stranding.

In addition to the extreme response of mass strandings, it is possible that lower levels of MFA sonar exposure could produce lesser behavioral reactions that could still have adverse effects on the whales. The greater variation in the $\Delta$ heading before the killer whale breakpoint likely represents standard foraging search patterns. These whales forage on deep dwelling prey items that may be located in discrete patches (Johnson et al. 2008), therefore they likely employ a foraging search pattern that maximizes their likelihood of encountering these patches. While we filtered out the smaller scale movements, the whales are still likely to move between feeding sites over the longer term. The light gray tracks in Figure 2 indicate the restricted area search typical for undisturbed beaked whales in the Tongue of the Ocean. The reduced variation in the $\Delta$ heading of the tagged whale after the killer whale break point indicates that it maintained a relatively straight course. Analysis of the acoustic record of the tag shows that the number of buzzes produced, which indicate prey cap ture events, was reduced during the sonar and killer whale playbacks and then increased in subsequent foraging dives (Tyack et al. 2011). These factors together may indicate that the whale was immediately reducing foraging effort in favor of directed flight from the area of playbacks. Areas with frequent sonar exercises may cause the resident population of beaked whales to abandon their preferred foraging habitat during sonar playbacks, possibly reducing their foraging intake or foraging selectivity (Tyack et al. 2011).

The whale reacted to a much lower received level for the killer whale playback than for the MFA sonar playback, however these stimuli were played in sequence so we cannot rule out the possibility that the effect of the playbacks was cumulative. Addi tionally, the AUTEC range is frequently used for naval sonar exercises including those utilizing MFA sonar signals. The repeated exposure to this signal may have habituated the tagged animal to these sounds, leading to the reduced reaction to the MFA playback. By contrast, killer whales are very rare in AUTEC waters. The calls of killer whales are likely a much less frequent sound heard at AUTEC than MFA sonar, so we cannot determine if the beaked whale recognized the sound as a potentially lethal predator, or whether it simply interpreted it as a novel sound, thus causing the stronger response to the killer whale playback. 
Two other factors make it possible that the killer whale playback stimulus could have been interpreted as a novel sound rather than recognized as killer whales. The killer whale vocalizations used for the playback stimulus were filtered to match the frequency range of the source, reducing the bandwidth considerably (Fig. S1). The recordings are also from the population of mammal eating killer whales residing in British Columbia, and therefore may differ from those of the whales in the Bahamas. We cannot disregard the possibility that these two alterations may have been signifi cant enough to change the whale's perception of the stimulus, from that of a preda tion call to simply a novel signal.

Additionally, while the Navy MFA sonar contains frequency and timing elements similar to that of killer whale predation calls, it is not an exact match. In the MFA playback, one $1.3 \mathrm{~s}$ MFA sonar sound was played every $25 \mathrm{~s}$, while the killer whale stimulus was an actual recordings of natural sounds, often with more than one vocali zation every $25 \mathrm{~s}$. However, both the MFA and killer whale sounds are below the best hearing range of those beaked whale species whose hearing has been measured (Cook et al. 2006). The lowered perception of signals in this frequency range may mean that the whales err on the side of caution and interpret the sonar signals in a natural behavioral context as similar to the sounds of a predator. The mismatch of some of the elements of the two signals may mean that the whales require either higher received levels or greater cumulative sound exposure levels in order to induce an anti predator reaction.

While it is not possible to draw a direct connection between MFA sonar and an antipredator behavioral reaction in $M$. densirostris due to the limited sample size and confounding factors, a definitive behavioral reaction has been quantified in this experiment. Despite the confounding factors, our results do show that Blainville's beaked whales respond to modified killer whale predation sounds with a prolonged and directed avoidance reaction. The method developed here can be applied to movement data from future controlled exposure experiments. Further experiments should focus on differentiating between the reactions to the two stimuli.

\section{ACKNOWLEDGMENTS}

The authors acknowledge the support and involvement of numerous field participants in this project. In particular, we acknowledge Leigh Hickmott who provided tagging support, and Walter Zimmer for analysis of tag data. In addition, we acknowledge Ian Boyd, Christo pher Clark, Diane Claridge, David Moretti, and Brandon Southall for their invaluable work conceiving of, planning, and executing this project. We thank Ari Daniel Shapiro for the initi ation of the data analysis. We also thank Volker Deecke for providing the recordings of killer whales used as a playback stimulus.

The authors acknowledge the support of the MASTS pooling initiative (The Marine Alli ance for Science and Technology for Scotland) in the completion of this study. MASTS is funded by the Scottish Funding Council (grant reference HR09011) and contributing institu tions. This research was conducted under permits for marine mammal research issued by the U.S. National Marine Fisheries Service to John Boreman (Permit 1121 1900) and to Peter Ty ack (Permit 81 1578), and issued by the Government of the Bahamas to Ian Boyd (Bahamas permit 02/07). The experiment was approved by the Institutional Animal Care and Use Com mittees of both Woods Hole Oceanographic Institution and the Bahamas Marine Mammal Research Organisation and the Animal Welfare and Ethics Committee of the University of $\mathrm{St}$ Andrews. 


\section{Literature Cited}

Amante, C., and B. W. Eakins. 2009. ETOPO1 1 arc minute global relief model: Procedures, data sources and analysis. NOAA Technical Memorandum NESDIS NGDC 24. 19 pp.

Berens, P. 2009. CircStat: A MATLAB toolbox for circular statistics. Journal of Statistical Software 31:25 27 .

Bryant, P. J., C. M. Lafferty and S. K. Lafferty. 1984. Reoccupation of Laguna Guerrero Negro, Baja California, Mexico, by gray whales. Pages 375387 in M. L. Jones, S. L. Swartz and S. Leatherwood, eds. The gray whale (Eschrichtius robustus). Academic Press, Orlando, FA.

Cao, R., and I. Van Keilegom. 2006. Empirical likelihood tests for two sample problems via nonparametric density estimation. Canadian Journal of Statistics 34:61 77.

Claridge, D. E. 2006. Fine scale distribution and habitat selection of beaked whales. M.Sc. thesis, University of Aberdeen, Aberdeen, Scotland. 238 pp.

Cook, M., R. Varela, J. Goldstein, et al. 2006. Beaked whale auditory evoked potential hearing measurements. Journal of Comparative Physiology A: Neuroethology, Sensory, Neural, and Behavioral Physiology 192:489 495.

Cox, T., T. Ragen, A. Read, et al. 2006. Understanding the impacts of anthropogenic sound on beaked whales. Journal of Cetacean Research and Management 7:177 187.

Crum, L. A., and Y. Mao. 1996. Acoustically enhanced bubble growth at low frequencies and its implications for human diver and marine mammal safety. The Journal of the Acoustical Society of America 99:2898 2907.

D'Amico, A., R. C. Gisiner, D. R. Ketten, J. A. Hammock, C. Johnson, P. L. Tyack and J. Mead. 2009. Beaked whale strandings and naval exercises. Aquatic Mammals 35:452 472 .

Deruiter, S. L., and A. R. Solow. 2008. A rotation test for behavioural point process data. Animal Behaviour 76:1429 1434.

Evans, D. L., and G. R. England. 2001. Joint Interim Report, Bahamas Marine Mammal Stranding Event of $1516 \mathrm{March} 2000.59$ pp. Available at http://www.nmfs. noaa.gov/ $\mathrm{pr} / \mathrm{pdfs} /$ health/stranding bahamas2000.pdf.

Finneran, J. J., D. S. Houser, B. Mase Guthrie, R. Y. Ewing and R. G. Lingenfelser. 2009. Auditory evoked potentials in a stranded Gervais' beaked whale (Mesoplodon europaeus). The Journal of the Acoustical Society of America 126:484 490 .

Fisher, N. I. 1995. Statistical analysis of circular data. Cambridge University Press, New York, NY.

Ford, J. K. B., and R. R. Reeves. 2008. Fight or flight: Antipredator strategies of baleen whales. Mammal Review 38:50 86.

Ford, J. K. B., G. M. Ellis, D. R. Matkin, K. C. Balcomb, D. Briggs and A. B. Morton. 2005. Killer whale attacks on minke whales: Prey capture and antipredator tactics. Marine Mammal Science 21:603 618

Frantzis, A. 1998. Does acoustic testing strand whales? Nature 392:29 29.

Jepson, P. D., M. Arbelo, R. Deaville, et al. 2003. Gas bubble lesions in stranded cetaceans. Nature 425:575 576 .

Johnson, M. P., and P. L. Tyack. 2003. A digital acoustic recording tag for measuring the response of wild marine mammals to sound. IEEE journal of oceanic engineering 28 : 312.

Johnson, M., P. T. Madsen, W. M. X. Zimmer, N. A. De Soto and P. L. Tyack. 2004. Beaked whales echolocate on prey. Proceedings of the Royal Society of London Series B: Biological Sciences 271:\$383 S386.

Johnson, M., L. S. Hickmott, N. Aguilar Soto and P. T. Madsen. 2008. Echolocation behaviour adapted to prey in foraging Blainville's beaked whale (Mesoplodon densirostris). Proceedings of the Royal Society Series B: Biological Sciences 275:133 139.

Manly, B. F. J. 2006. Randomization, bootstrap and monte carlo methods in biology. Second edition. Taylor \& Francis, Boca Raton, FL. 
Marques, T. A., L. Thomas, J. Ward, N. Dimarzio and P. L. Tyack. 2009. Estimating cetacean population density using fixed passive acoustic sensors: An example with Blainville's beaked whales. The Journal of the Acoustical Society of America 125:1982-1994.

McDonald, M. A., J. A. Hildebrand, S. M. Wiggins and D. Ross. 2008. A 50 year comparison of ambient ocean noise near San Clemente Island: A bathymetrically complex coastal region off Southern California. The Journal of the Acoustical Society of America 124:1985-1992.

Parks, S. E., C. W. Clark and P. L. Tyack. 2007. Short- and long-term changes in right whale calling behavior: The potential effects of noise on acoustic communication. The Journal of the Acoustical Society of America 122:3725-3731.

Tyack, P. L., M. Johnson, N. Aguilar De Soto, A. Sturlese and P. T. Madsen. 2006. Extreme diving of beaked whales. The Journal of Experimental Biology 209:4238-4252.

Tyack, P. L., W. M. X. Zimmer, D. Moretti, et al. 2011. Beaked whales respond to simulated and actual navy sonar. PLoS ONE 6:e17009.

Ward, J., R. Morrissey, D. Moretti, et al. 2008. Passive acoustic detection and localization of Mesoplodon densirostris (Blainville's beaked whale) vocalizations using distributed bottommounted hydrophones in conjunction with a Digital Tag (DTag) recording. Canadian Acoustics 36:60-66.

Zimmer, W. M. X., and P. L. Tyack. 2007. Repetitive shallow dives pose decompression risk in deep-diving beaked whales. Marine Mammal Science 23:888-925.

Zimmer, W. M. X., M. P. Johnson, P. T. Madsen and P. L. Tyack. 2005. Echolocation clicks of free-ranging Cuvier's beaked whales (Zipbius cavirostris). The Journal of the Acoustical Society of America 117:3919-3927.

Received: 5 November 2012

Accepted: 1 February 2013

\section{SUPPORTING INFORMATION}

The following supporting information is available for this article online at http:/ onlinelibrary.wiley.com/doi/10.1111/mms.12028/suppinfo.

Figure S1. Spectrograms of a $17 \mathrm{~s}$ segment of the killer whale playback stimulus. Top: Spectrogram of the unfiltered killer whale vocalizations. Bottom: Spectrogram of the killer whale vocalizations filtered to between 2 and $5 \mathrm{kHz}$.

Figure S2. Comparison of rotated and observed nonparametric likelihood-ratios (NLR) calculated for the MFA sonar playback breakpoint. The histogram shows the distribution of the NLR calculated for the rotated data order, and the observed NLR is plotted in red. It is exceeded by 146 of the rotated NLR values, giving a $P$-value of 0.468 . 


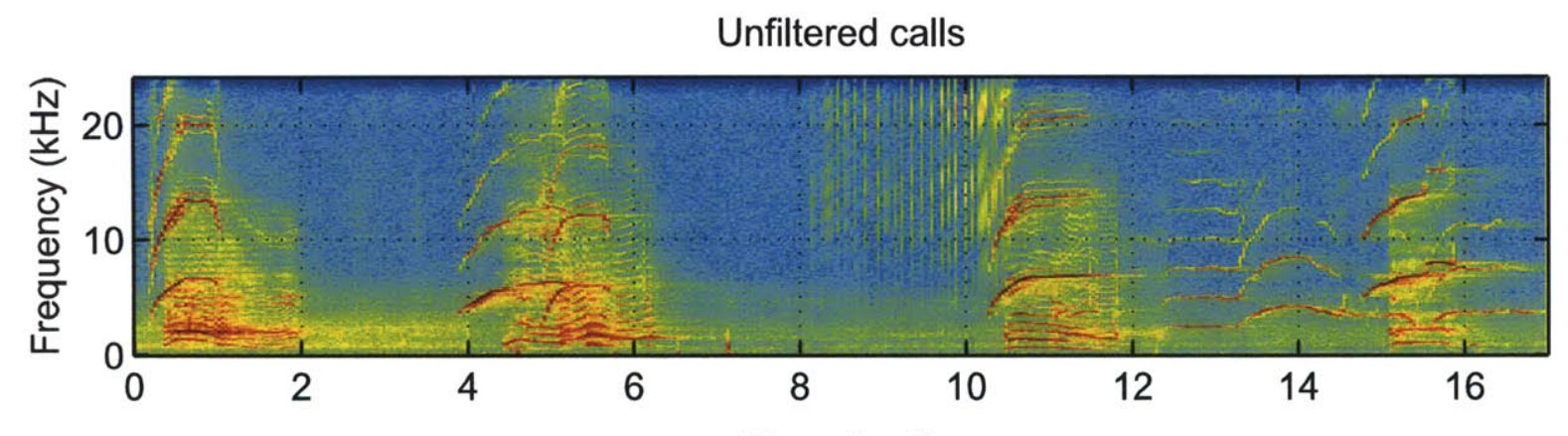

Filtered calls

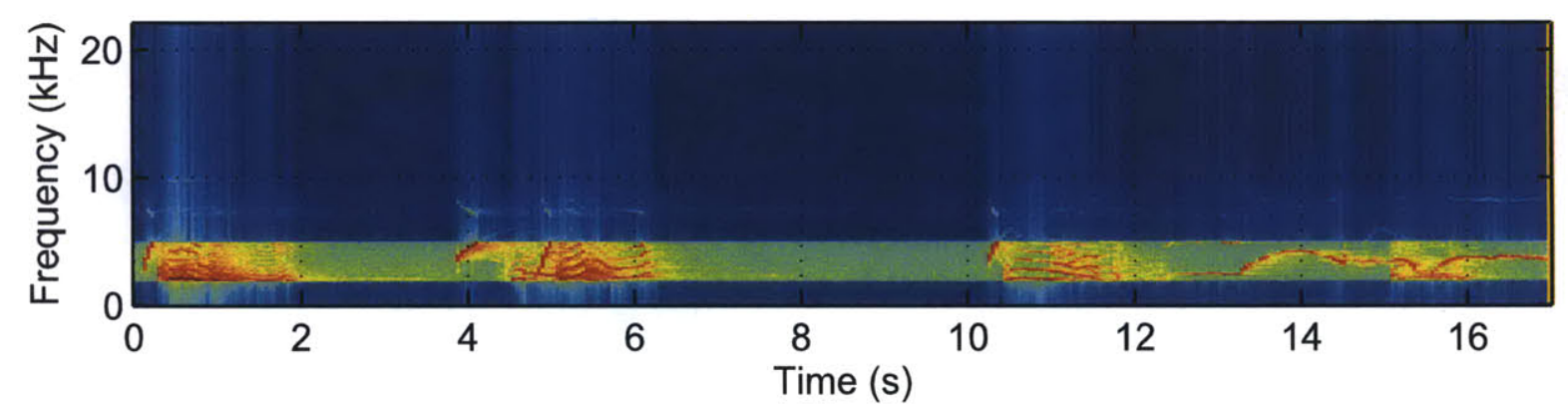

Figure S1: Spectrograms of a $17 \mathrm{~s}$ segment of the killer whale playback stimulus. Top: Spectrogram of the unfiltered killer whale vocalizations.

Bottom: Spectrogram of the killer whale vocalizations filtered to between 2 and $5 \mathrm{kHz}$ 


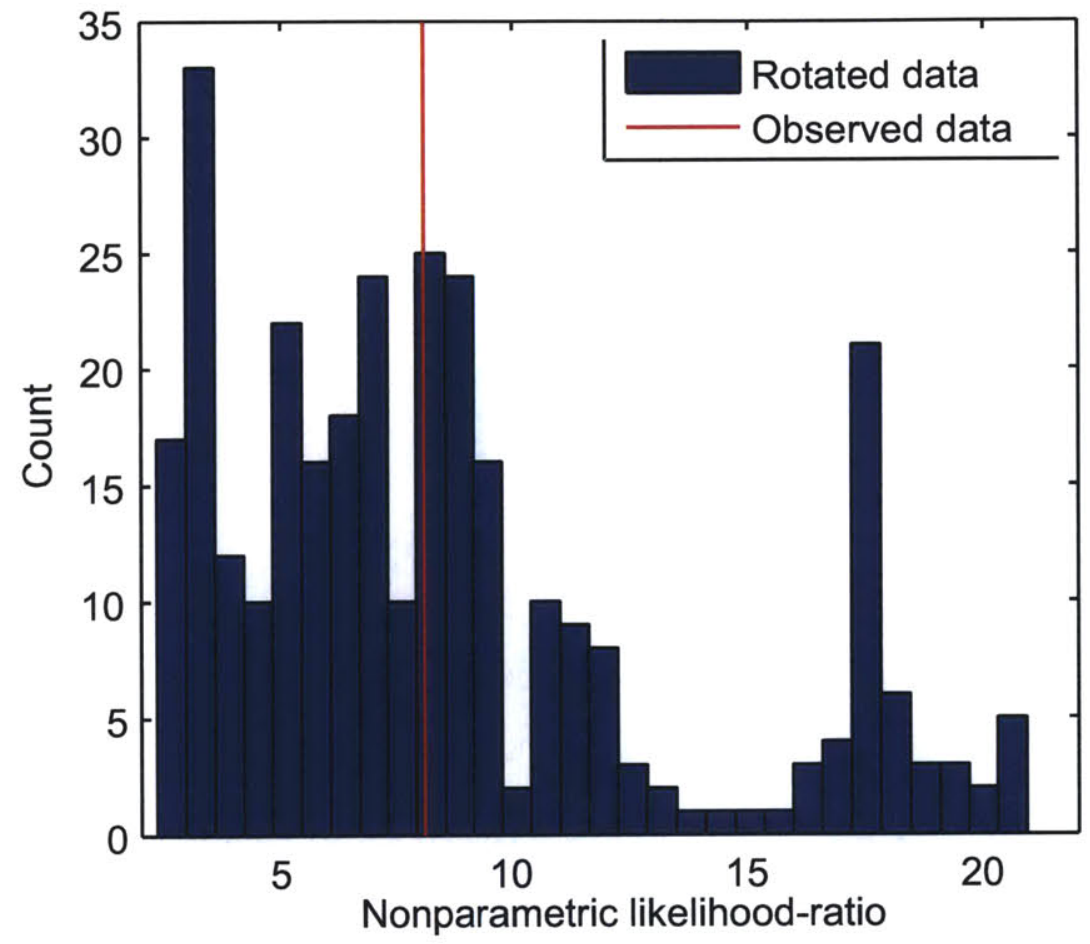

Figure S2: Comparison of rotated and observed nonparametric likelihoodratios (NLR) calculated for the MFA sonar playback breakpoint. The histogram shows the distribution of the NLR calculated for the rotated data order, and the observed NLR is plotted in red. It is exceeded by 146 of the rotated NLR values, giving a P-value of 0.468 . 


\section{Chapter 5}

Conclusions 


\section{Conclusions}

This thesis has taken a first look at the roles of geomagnetic and acoustic cues in humpback whale navigation and orientation, as well as documented some of the effects of human-induced sound on beaked whales. It is evident that migrating whales employ a sophisticated navigation system to make their way between breeding and feeding grounds. However, the size and mobility of these animals makes it extremely difficult to conduct manipulative experiments that can conclusively show the use of specific navigation and orientation cues. A combination of advances in tracking and tagging technology and judicious use of coastally migrating whales have allowed for some initial correlations to be established here.

Chapter 2 examined the migration tracks of humpback whales satellite-tagged in Hawaii, investigating the role that geomagnetic cues play in determining their migration routes. Applying the methods of Benhamou (2003), a route between each whale's start and end location was modeled, based solely on navigation via geomagnetic intensity and inclination. In seven out of nine tracks, the direction of the deviation from the great circle route matched between the observed and modeled migration routes. According to a binomial test, the P-value for this proportion was $\sim 0.09$, which is not significant at the conventional 0.05 alpha level. However, the power of the test is limited by the small sample size; doubling the sample size would allow for a more powerful test of the hypothesis. Examination of the parameters of the model revealed that the paths modeled for each whale would only be observed under the very specific combination of the geomagnetic inclination and intensity field parameters with the home direction. These considerations lend weight to the argument that the high percentage of correlated deviation directions may be due to more than just chance.

While the deviation direction matches in many cases, the magnitude of the deviation is always greater in the modeled track compared to the observed track. This is consistent with the prediction that the whales are likely using multiple sources of orientation information to navigate, as integration of other cues could allow for the improved accuracy seen in the observed whale tracks. This multi-cue integration, 
performed both sequentially and in parallel, has been found in other migratory species (Lohmann and Lohmann 1996, Wiltschko and Wiltschko 2003, Bingman and Cheng 2005). The utilization of multiple cues makes evolutionary and ecological sense, as different cues operate on varying spatial scales and are all subject to fluctuations in availability and accuracy. Without multiple sources of orientation information, a migrating animal would be extremely vulnerable when the preferred cue is unavailable or disrupted.

While there have been many studies of other animals showing a geomagnetic sense, there have thus far been no studies examining the exact methods an animal could use to navigate via a bicoordinate map using these cues. This study provided a first look at the applicability of the theoretical methods of Benhamou (2003), the only comprehensive model of bicoordinate navigation methods to date. When the 'complex' route, which involves taking two non-orthogonal gradient fields into account conjointly, was modeled for each track using geomagnetic inclination and intensity, it was found not to be applicable to migrations in the North Pacific. Conversely, the method that Benhamou (2003) suggested as conceptually simple for an animal to perform proved to be both applicable and useful for geomagnetic inclination and intensity in the North Pacific. This suggests that a method in which an animal takes each field gradient into account separately may not only be the simpler navigation solution, but also the more widely applicable method.

Future work should focus on collecting more satellite tracks of migrating whales. Of particular interest would be tracks of animals migrating from Alaska to Hawaii, as this would solve much of the uncertainty in destination, as well as demonstrating how well the whales are able to locate the precise location of an island destination. Additionally, increasing the sample size of migration tracks would allow for more conclusive testing of whether or not the matching deviation directions are due purely to chance. This same method could also be applied to satellite tracks from other species of whales, or to whales in different areas of the world where the geomagnetic fields differ. If the same pattern observed here emerged in such cases it would lend considerable strength to the 
hypothesis that whales navigate with the aid of geomagnetic cues. Additional work should also focus on improving the bicoordinate model by methods such as adjusting the rate at which the subject crosses each field, adding a compass bearing to the geomagnetic cues, or expanding the model into a general inverse problem by adding navigation cues until the modeled track approximates the observed tracks. When multiple models are established to generate alternative route, empirical methods should be utilized to determine which model most closely approximates the observed migration routes. In this manner, migration tracks of any animal species could be used to examine the navigation methods it employs.

Chapter 3 examined the use of acoustic signals as an orientation cue during nearshore migrations of humpback whales. An attempt was made to quantify the sounds of waves crashing on the island, and assess the potential of these sounds to act as an indicator of the island's presences, the acoustic records failed to show any consistent signal. However, acoustic recordings did show a strong, consistent sound signal from snapping shrimp on the island. Acoustic propagation calculations demonstrated that the sounds of the snapping shrimp would have been audible past the $2 \mathrm{~km}$ visual survey range of this study. The far-ranging and consistent nature of these snapping shrimp sounds suggests that they may be well suited as a cue for obstacle detection and avoidance. Additionally, there were no discernible differences in the approach behavior of the whales during different assumed sound conditions as they approached a rocky island. This further supports the conclusion that the whales can detect the island at distances well beyond those needed for a reaction and the island therefore poses no danger of collision.

Future studies should focus on assessing the frequencies and levels of sounds from waves crashing on a rocky island. This accomplishment would likely require the use of a bottom-mounted hydrophone, or a drifter, which would limit any self-noise on the recordings that could mask the sound signals, particularly at low frequencies. In addition, long term recordings at varying ranges from an island would allow for more precise quantification of the sound field a whale experiences as it approaches. If the 
sounds levels, transmission loss, and noise levels can be accurately measured, then the detection range of all sound signals from a rocky island could be calculated.

Additionally, playbacks of the combination of island sounds in the otherwise unobstructed path of migrating whales would help to indicate whether or not the whales detect and recognize the cues as indicating an obstacle's presence. The potential for sound to propagate long distances in the ocean, and the use of acoustic signals as orientation cues by larvae (Simpson et al. 2005), indicate that sound could be an important orientation cue in the ocean.

Chapter 4 demonstrates why understanding the role of sound in marine mammal ecology is important for conservation. Navy mid-frequency active sonar has been linked to the stranding deaths of beaked whales of several different species. The study was designed to determine whether or not beaked whales are mistaking Navy sonar signals for the predation calls of killer whales, and initiating an anti-predator response that puts them at risk of stranding. In this study, the sequential playback of Navy sonar signals and killer whale predation calls to a tagged Blainville's beaked whale elicited an extended avoidance reaction. While the whale had a strong reaction only after playback of the killer whale calls, the fact that the signals were played in sequence means that a cumulative effect cannot be ruled out. Regardless, a strong avoidance reaction by the tagged animal was seen in response to the playbacks. Beaked whales dwell in deep water, so a prolonged avoidance reaction in which they swim away from these deep water habitats could put them at risk for stranding. If a beaked whale swims quickly away from a sonar playback it could be at risk of stranding due to an intentional last-ditch evasion strategy, or possibly an error in navigation while fleeing.

Future studies on this subject should focus on separating the effects of playback of navy sonar and killer whale predation calls on beaked whales. Additionally, if the extended avoidance can be attributed to a predator avoidance response, and shown to pose a risk for stranding, the elements of the sonar stimulus that elicit the response should be investigated. 


\section{Significance}

The problem of how migratory animals find their way has long been a subject of curiosity and investigation. Examination of animal navigational abilities has uncovered a whole new suite of sensory mechanisms, the most prominent of which is the magnetic sense discovered in a large number of species. While the problem of which senses a marine mammal uses to navigate and orient in the ocean is a difficult one to address, some basic insights have been established here, and it is important that this avenue of investigation be continued.

Understanding how whales navigate is important not just for the intellectual merits of such information, but for the potential conservation benefits inherent in understanding the sensory ecology of these whales. Norris (1967) argued that a failure of navigation mechanisms is the cause of mass strandings of otherwise healthy animals, and it has been suggested that mass strandings of animals occur in certain environments because of distortion in the echo signals that the cetaceans are using for navigation (Brabyn and McLean 1992), or because of magnetic anomolies in the area (Klinowska 1986). If navigational mechanisms can be elucidated in whales, and errors in these systems of navigation linked to mass strandings, then new methods could potentially be developed to correct these errors and prevent future stranding events.

Identifying acoustics as an important part of whale navigation would have additional conservation impacts. It has long been known that increasing levels of lowfrequency anthropogenic noise in the oceans can negatively affect cetaceans. Human contributions to sound in the ocean, from shipping, oil and gas development, and military activities, have greatly increased over the last 50 years (Croll et al. 2001, Hildebrand 2009). There have been multiple attempts to regulate human-produced noise in the marine environment, but public agencies encounter difficulties because of a scarcity of data on the problem (Southall et al. 2007). Therefore, any new information on how whales utilize sound, in myriad aspects of their lives, can greatly aid in informing regulations on anthropogenic sound in the ocean. 
In conclusion, this thesis has taken one of the first looks at the question of whale navigation. Up to this point almost nothing has been known about how these animals make their way across the oceans in their annual migration. While it is very difficult to conclusively prove the use of any specific navigation cue, the plausibility of geomagnetic and acoustic cues has been investigated here. A method by which an animal could use bicoordinate geomagnetic navigation in the North Pacific has been established, as well as an initial investigation of the possibility that this method is being used by migrating humpback whales. Additionally, snapping shrimp sounds have been shown to provide a very loud, long ranging sound cue that could indicate the presence of rocky islands or other hard substrates, and thus aid in whale orientation in the near-shore environment. Lastly, the potential reason behind one of the most dramatic effects of anthropogenic sounds, the stranding of beaked whales, was investigated, and a prolonged avoidance reaction in response to playback of killer whale sounds established. In summation, this thesis has established some initial avenues of investigation into whale navigation and orientation and will hopefully provide the basis for future research on the subject.

\section{References}

Benhamou, S. 2003. Bicoordinate navigation based on non-orthogonal gradient fields. Journal of theoretical biology 225:235-239.

Bingman, V. P. and K. Cheng. 2005. Mechanisms of animal global navigation: Comparative perspectives and enduring challenges. Ethology, ecology \& evolution 17:295.

Brabyn, M. W. and I. G. McLean. 1992. Oceanography and coastal topography of herdstranding sites for whales in new zealand. Journal of Mammalogy 73:469-476.

Croll, D. A., C. W. Clark, J. Calambokidis, W. T. Ellison and B. R. Tershy. 2001. Effect of anthropogenic low-frequency noise on the foraging ecology of balaenoptera whales. Animal Conservation 4:13-27.

Hildebrand, J. A. 2009. Anthropogenic and natural sources of ambient noise in the ocean. Marine Ecology Progress Series 395. 
Klinowska, M. 1986. Cetacean live stranding dates relate to geomagnetic disturbances. Aquatic Mammals 11:109.

Lohmann, K. and C. Lohmann. 1996. Orientation and open-sea navigation in sea turtles. Journal of Experimental Biology 199:73-81.

Norris, K. S. 1967. Some observations on the migration and orientation of marine mammals. Pages 101-125 R. M. Storm ed.in Animal orientation and navigation. Corvallis: Oregon State University Press.

Simpson, S. D., M. Meekan, J. Montgomery, R. McCauley and A. Jeffs. 2005. Homeward sound. Science 308:221-221.

Southall, B. L., A. E. Bowles, W. T. Ellison, J. J. Finneran, R. L. Gentry, C. R. Greene, D. Kastak, D. R. Ketten, J. H. Miller, P. E. Nachtigall, W. J. Richardson, J. A. Thomas and P. L. Tyack. 2007. Marine mammal noise exposure criteria. Aquatic Mammals 33.

Wiltschko, R. and W. Wiltschko. 2003. Avian navigation: From historical to modern concepts. Animal Behaviour 65:257-272. 